\title{
Large deviations for a class of nonhomogeneous Markov chains
}

\author{
Zach Dietz and Sunder Sethuraman \\ Tulane University and Iowa State University
}

October 18, 2018

\begin{abstract}
Large deviation results are given for a class of "perturbed" nonhomogeneous Markov chains on finite state space which formally includes some stochastic optimization algorithms. Specifically, let $\left\{P_{n}\right\}$ be a sequence of transition matrices on a finite state space which converge to a limit transition matrix $P$. Let $\left\{X_{n}\right\}$ be the associated nonhomogeneous Markov chain where $P_{n}$ controls movement from time $n-1$ to $n$. The main statements are a large deviation principle and bounds for additive functionals of the nonhomogeneous process under some regularity conditions. In particular, when $P$ is reducible, three regimes depending on the decay of certain "connection" $P_{n}$-probabilities are identified. Roughly, if the decay is too slow, too fast, or in an intermediate range, the large deviation behavior is trivial, same as the time-homogeneous chain run with $P$, or non-trivial and involving the decay rates. Examples of anomalous behaviors are also given when the approach $P_{n} \rightarrow P$ is irregular. Results in the intermediate regime apply to "geometrically fast" running optimizations, and to some issues in glassy physics.

Research supported in part by NSF grant NSF/DMS-0003811.

Key words and phrases: large deviations, nonhomogeneous, Markov, optimization, geometric cooling, glassy models.

Abbreviated title: LDP for some nonhomogeneous Markov chains.

AMS (2000) subject classifications: Primary 60J10; secondary 60F10.

\section{Introduction}

The purpose of this paper is to provide some large deviation bounds and principles for a class of nonhomogeneous Markov chains related to some popular stochastic optimization algorithms such as Metropolis and simulated annealing schemes. In a broad sense, these algorithms are stochastic perturbations of steepest descent or "greedy"
\end{abstract}


procedures to find the global minimum of a function $H$, and are in the form of nonhomogeneous Markov chains whose connecting transition kernels converge to a limit kernel associated with steepest descent.

For instance, in the Metropolis algorithm on finite state space $\Sigma$, the transition kernel connecting times $n-1$ and $n$ is given by

$$
P_{n}(i, j)=\left\{\begin{array}{rr}
g(i, j) \exp \left\{-\beta_{n}(H(j)-H(i))_{+}\right\} & \text {for } j \neq i \\
1-\sum_{l \neq i} P_{n}(i, l) & \text { for } j=i
\end{array}\right.
$$

where $g$ is an irreducible transition function, and $\beta_{n}$ represents an "inverse temperature" parameter which diverges, $\beta_{n} \rightarrow \infty$. Here, the limit kernel $P=\lim _{n} P_{n}$ corresponds to steepest descent in that jumps from $i$ to $j$ when $H(j)>H(i)$ is not allowed.

These type of schemes are intensively used in image analysis (cf. Winkler [35]), neural networks (cf. Bovier and Picco eds. [5]), statistical physics of glassy systems, combinatorial optimizations, etc. (cf. Salamon et al. [27]). More general tutorials include Catoni [6], van Laarhoven and Aarts [22], Geman [15], and Gidas [16].

Virtually all previous large deviations work with respect to optimization chains has been through Freidlin-Wentzell type methods [13]. This approach is to consider a sequence of time-homogeneous Markov chains, parametrized by temperature, which approaches the steepest descent chain as the temperature cools, and then to transfer "short time" large deviation estimates to a single related system in which temperature varies with time. For instance, with respect to the Metropolis algorithm, by studying the sequence of time-homogeneous chains $\left\{X^{\beta}: \beta \geq 0\right\}$ where $\beta_{n} \equiv \beta$ and $\beta \uparrow \infty$, estimates can be made on the nonhomogeneous chain where $\beta_{n}$ varies. Although this approach has had much success, especially related to statistical physics "metastability" questions, it seems however that only large deviation bounds are recovered for the position of the nonhomogeneous process rather than large deviation principles (LDP's) (See Catoni [6], [8], Catoni and Cerf [9], and Bovier et al. [2], Bovier and Gayard [3], and references therein). It would be then natural to ask about LDP's for empirical averages which are more regular objects than the positions.

In a different, more general vein, LDP's have been shown for independent nonidentically distributed variables whose Cesaro empirical averages converge (Seppäläinen [30]), and also for some types of Gibbs measures which include nonhomogeneous chains whose connecting transition kernels are positive entrywise and converge in Cesaro mean to a positive limit matrix (Seppäläinen [32]).

Other work in the literature treat an intermediate case of nonhomogenity, namely Markov chains whose transition kernels are chosen at random from a time-homogeneous process. The results here are then to prove an LDP for almost all realized nonhomogeneous Markov chains chosen in this fashion (Seppäläinen [31] and Kifer [20]). Also, we note that an LDP has been shown for a class of "near" irreducible time-homogeneous processes satisfying some mixing conditions (Baxter et al. [1]).

In this context, we develop here an LDP in natural scale $n$ with explicit rate function for the empirical averages of nonhomogeneous Markov chains on finite state spaces 
whose transition kernels converge to the general limit matrix which allows for reducibility, a key concern in optimization schemes. We note the methods used here differ from Freidlin-Wentzell type arguments in that they focus on the nonhomogeneous process itself rather than homogeneous approximations. The specific techniques used are constructive and involve various "surgeries" of path realizations and some coarse-graining.

Let $\Sigma=\{1,2, \ldots, \mathfrak{r}\}$ be a finite set of points. Let $P_{n}=\left\{p_{n}(i, j): i, j \in \Sigma\right\}$ be a sequence of $\mathfrak{r} \times \mathfrak{r}$ stochastic matrices for $n \geq 1$, and $\pi$ be a distribution on $\Sigma$. Let now $\mathbb{P}_{\pi}=\mathbb{P}_{\pi}^{\left\{P_{n}\right\}}$ be the (nonhomogeneous) Markov measure on the sequence space $\Sigma^{\infty}$ with Borel sets $\mathcal{B}\left(\Sigma^{\infty}\right)$ corresponding to initial distribution $\pi$ and transition kernels $\left\{P_{n}\right\}$. That is, with respect to the coordinate process, $X_{0}, X_{1}, \ldots$, we have the Markov property

$$
\mathbb{P}_{\pi}\left(X_{n+1}=j \mid X_{0}, X_{1}, \ldots, X_{n-1}, X_{n}=i\right)=p_{n+1}(i, j)
$$

for all $i, j \in \Sigma$ and $n \geq 0$. We see then that $P_{n+1}$ controls "transitions" between times $n$ and $n+1$.

We now specify the class of nonhomogeneous processes focused upon in the article. Let $\pi$ be a distribution and $P=\{p(i, j)\}$ be a stochastic matrix on $\Sigma$. Define the collection $\mathbb{A}(P)$ by

$$
\mathbb{A}(P)=\left\{\mathbb{P}_{\pi}^{\left\{P_{n}\right\}}: P_{n} \rightarrow P\right\}
$$

where the convergence $P_{n} \rightarrow P$ is elementwise, that is $\lim _{n \rightarrow \infty} p_{n}(i, j)=p(i, j)$ for all $i, j \in \Sigma$. The collection $\mathbb{A}$ can be thought of as perturbations of the time-homogeneous Markov chain run with $P$, and is a natural class in which to explore how "nonhomogeneity" enters into the large deviation picture.

We also remark that this class has been studied in connection with other types of problems such as ergodicity (Isaacson and Madsen [19]), laws of large numbers (Wen and Weiguo [34] and Winkler [35]), and fluctuations (Hanen [17]). See also Miclo [25] and Gaudron and Trouve [14] for some laws of large numbers and fluctuation results for "generalized annealing algorithms" and Markov chains with rare transitions.

Let now $f: \Sigma \rightarrow \mathbb{R}^{d}$ be a $d \geq 1$ dimensional function. Let also $\mathbb{P}_{\pi} \in \mathbb{A}(P)$ be a " $P$-perturbed" nonhomogeneous Markov measure. In terms of the coordinate process, define the additive sum $Z_{n}=Z_{n}(f)$ for $n \geq 1$ by

$$
Z_{n}=\frac{1}{n} \sum_{i=1}^{n} f\left(X_{i}\right) .
$$

The specific goal of the paper is to understand the large deviation behavior of the induced distributions of $\left\{Z_{n}: n \geq 1\right\}$ with respect to $\mathbb{P}_{\pi}$ in scale $n$. That is we search for a rate function $\mathbb{J}$ so that for Borel sets $B \subset \mathbb{R}^{d}$,

$$
-\inf _{z \in B^{o}} \mathbb{J}(z) \leq \underline{\lim } \frac{1}{n} \log \mathbb{P}_{\pi}\left(Z_{n} \in B\right) \leq \varlimsup \lim \frac{1}{n} \log \mathbb{P}_{\pi}\left(Z_{n} \in B\right) \leq-\inf _{z \in \bar{B}} \mathbb{J}(z) .
$$

An immediate question which comes to mind is whether these large deviations for the nonhomogeneous chain, if they exist, differ from the deviations with respect to the 
time-homogeneous chain run with $P$. The general answer found in our work is "yes" and "no," and as might be suspected depends on the rate of convergence $P_{n} \rightarrow P$ and the structure of the limit matrix $P$.

More specifically, when $P$ is irreducible, it turns out that the large deviation of behavior of $\left\{Z_{n}\right\}$ under $\mathbb{P}_{\pi}$ is the same as that under the time-homogeneous chain associated with $P$ and independent of the rate of convergence of $P_{n}$ to $P$. (Note [32] covers the case $P$ is positive entrywise, and [30] the case when each $P_{n}$ has identical rows.)

Perhaps, the more interesting case is when the target matrix $P$ is reducible. Indeed, this is the case with stochastic optimization algorithms where $H$ has several local minima, e.g. with respect to the Metropolis process, the local minima sets of $H$ do not communicate in the limit steepest descent chain. In this situation, the large deviations of $\left\{Z_{n}\right\}$ depend both on the type of reducibilities of $P$, and the decay rate, with respect to $P_{n}$, of certain "connection probabilities" between $P$-irreducible sets, and fall into three categories. Namely, when the decay is "fast," or super exponential, the large deviation behavior is the same as for the time-homogeneous Markov chain run under $P$; when the speed is "slow," or sub-exponential, one obtains a "trivial" large deviation behavior; and finally, when the speed is "intermediate," or when the "connection probabilities" are on the order $e^{-C n}$, a non-trivial behavior is found which differs from stationarity.

We remark now, in terms of applications, the "intermediate" processes are important in situations such as (i) "fast annealing" simulations, and (ii) models of glass formation. (i) In Metropolis-type procedures, classic convergence theorems mandate that the temperatures satisfy $\beta_{n} \leq C_{H} \log n$ with respect to a known constant $C_{H}$ for the process to converge to the global minima set of $H$ (cf. Catoni [6], Gidas [16]),

$$
\lim _{n \rightarrow \infty} \mathbb{P}_{\pi}\left(X_{n} \in \text { global minima set of } H\right)=1 .
$$

However, with only finite time and resources, the optimal logarithmic speed is too slow to yield good results. In fact, in violation of classic results, exponentially fast schemes where $\beta_{n} \sim n$ are often used for which the process may actually converge to a nonglobal but local minimum of $H$. As "connecting probabilities" between local minima sets are on order $e^{-C \beta_{n}}$, these chains fit naturally in the "intermediate" framework mentioned above (cf. discussion after Corollary 3.1). Although there are some good error bounds for these "geometrically cooling" experiments in finite time (cf. Catoni [7]), it seems the structure of the associated dynamics is not that well understood however (cf. section $6.2[35]$ ).

(ii) In the manufacture of glass, a hot, fired material is quickly quenched into a substance which is not quite solid nor liquid. The interpretation is that under rapid cooling the constructed glass is caught in a local energy optimum associated with some spatial disorder-not the regularly structured global one associated with a "solid"-from which over much longer time scales it may move to other states (cf. Lewis [23]). Such glassy systems are intensively studied in the literature. Two rough concerns can be 
identified: What are the typical glass landscapes which specify the local optima, and what are the dynamics of the quick quenching phase and beyond. Much discussion is focused on the first concern (cf. Schon and Sibani [28]). But, even in systems where statics are quantified, dynamical questions remain open (cf. Mezard [24], part IV [27]). However, with respect to "metastability," as mentioned earlier, much work has been accomplished (cf. Bovier and Manzo [4], den Hollander et al. [18], Vares [33] and references therein). Less work has been done though when certain time inhomogeneities are severe, say on exponential scale $e^{-C n}$, in the context of Metropolis models in the "intemediate" regime.

At this point, we observe, as alluded to above in the two examples, that (from BorelCantelli arguments) the typical large scale picture of general "intermediate" speed nonhomogeneous Markov chains is to get trapped in one of the irreducible sets corresponding to the limit $P$, e.g. the local $H$-minima sets in the Metropolis scheme. In this sense, the large deviations rate function $\mathbb{J}$, found with respect to averages $\left\{Z_{n}\right\}$, is relevant in understanding how atypical deviations arise, namely how the process average can "survive" for long times, that is how $Z_{n} \sim z$ for large $n$ when $z$ is not an $P$-irreducible set average. More specifically, when $P$ corresponds to $K \geq 2$ irreducible sets $\left\{C_{\zeta_{j}}\right\}$, we show $\mathbb{J}$ is an optimization between two types of costs, and is in form

$$
\begin{aligned}
\mathbb{J}(z)=\min _{\sigma \in \mathbb{S}} \inf _{\vec{v} \in \Omega} \inf _{\vec{x} \in D(\vec{v}, z)} \\
\quad-\sum_{i=1}^{K-1}\left(\sum_{j=1}^{i} v_{j}\right) \mathcal{U}\left(\zeta_{\sigma(i)}, \zeta_{\sigma(i+1)}\right)+\sum_{i=1}^{K} v_{i} \mathbb{I}_{\zeta_{\sigma(i)}}\left(x_{i}\right) .
\end{aligned}
$$

Here, $\mathbb{I}_{\zeta_{j}}$ is the rate function for the $P$-time-homogeneous chain restricted to $C_{\zeta_{j}}$ and represents a "resting" cost of moving within $C_{\zeta_{j}}$, and $\mathcal{U}\left(\zeta_{j}, \zeta_{k}\right)$ is a large deviation "routing" cost of traveling between $C_{\zeta_{j}}$ and $C_{\zeta_{k}}$. Also, $\mathbb{S}$ and $\Omega$ are the sets of permutations and probabilities on $\{1,2, \ldots, K\}$ respectively, and $D(\vec{v}, z)$ is the set of vectors $\vec{x}$ such that $\sum_{j=1}^{K} v_{j} x_{j}=z$. The intuition then is that $Z_{n}$ optimally deviates to $z$ by visiting sets $\left\{C_{\zeta_{j}}\right\}$ finitely many times, in a certain order $\sigma$ with time proportions $\vec{v}$, so that the average $z$ is maintained, and resting and routing costs are minimized.

Our main theorem (Theorem 3.3) is that under some natural regularity conditions on the approach $P_{n} \rightarrow P$, the average $Z_{n}$ satisfies an LDP with rate function $\mathbb{J}$. When $\mathcal{U} \equiv-\infty$ or $\mathcal{U} \equiv 0$, that is when "connection probabilities" vanish too fast or too slow, the rate $\mathbb{J}$ reduces to the rate function for the time-homogeneous chain run under $P$ or a trivial one. When the "connections" are exponential, $-\infty<\mathcal{U}<0$ and $\mathbb{J}$ nontrivially incorporates the convergence exponents (Corollary 3.1). Some comments to the Metropolis algorithm are made at the end of section 3. When the approach is irregular, large deviation bounds (Theorems 3.1 and 3.2) and examples (section 12) of anomalous behaviors are also given.

Finally, it is natural to ask about the large deviations on scales $\alpha_{n}$ different from scale $n$, that is the values of the limits $\varliminf\left(1 / \alpha_{n}\right) \log \mathbb{P}_{\pi}\left(Z_{n} \in B\right)$. The meta-result 
should be, if the typical system behavior is to be absorbed into certain sets, the analogous large deviation behavior holds in scale $\alpha_{n}$ with revised "resting" and "routing" costs reflecting the scale. In fact, with respect to the Metropolis model, by the methods in this article, large deviation bounds and principles in scale $\beta_{n}$ can be derived so long as $\underline{\lim } \beta_{n} / n^{\theta}=\infty$ for some $\theta>0$. In principle, similar results should hold when $\beta_{n}>C \overline{\log n}$ and $C>1$, although this is not pursued here. On the other hand, large deviation principles in scale $\beta_{n} \leq \log n$ are of a completely different category as in this case there is no local minima absorbtion (see however [9] for LD bounds with respect to metastability concerns).

\section{Preliminaries}

We now recall and develop some definitions and notation before arriving at the main theorems. Throughout, we will make the convention that $\pm \infty \cdot 0=0$ and $\log 0=-\infty$.

Rate Functions and Extended LDP. Let $\mathbb{I}: \mathbb{R}^{d} \rightarrow \mathbb{R} \cup\{\infty\}$ be an extended realvalued function. We say that $\mathbb{I}$ is a extended rate function if $\mathbb{I}$ is lower semi-continuous, and further that $\mathbb{I}$ is a good extended rate function if in addition the level sets of $\mathbb{I}$, namely $\{x: \mathbb{I}(x) \leq a\}$ for $a \in \mathbb{R}$, are compact. This definition extends the usual notion of rate function where negative values are not allowed (cf. Section 1.2 [10]). Namely, we say $\mathbb{I}$ is a (good) rate function if $\mathbb{I}: \mathbb{R}^{d} \rightarrow[0, \infty]$ is a (good) extended rate function.

We denote $Q_{\mathbb{I}} \subset \mathbb{R}^{d}$ as the domain of finiteness, $Q_{\mathbb{I}}=\left\{x \in \mathbb{R}^{d}: \mathbb{I}(x)<\infty\right\}$. We also recall the standard notation for $B \subset \mathbb{R}^{d}$ that $\mathbb{I}(B)=\inf _{x \in B} \mathbb{I}(x)$.

Let now $\left\{\mu_{n}: n \geq 1\right\}$ be a sequence of non-negative measures with respect to Borel sets on $\mathbb{R}^{d}$. We say that $\left\{\mu_{n}\right\}$ satisfies an large deviation principle with (extended) rate function $\mathbb{I}$ if for all Borel sets $B \subset \mathbb{R}^{d}$ we have

$$
-\inf _{x \in B^{o}} \mathbb{I}(x) \leq \underline{\lim } \frac{1}{n} \log \mu_{n}(B) \leq \varlimsup \frac{1}{n} \log \mu_{n}(B) \leq-\inf _{x \in \bar{B}} \mathbb{I}(x) .
$$

Non-Negative Matrices. Let $U=\{u(i, j)\}$ be a matrix on $\Sigma$ and let $C \subset \Sigma$ be a subset of states. Define $U_{C}=\{u(i, j): i, j \in C\}$ as the corresponding submatrix. We say that $U_{C}$ is non-negative, denoted $U_{C} \geq 0$, if all entries are non-negative. Analogously, $U_{C}$ is positive, denoted $U_{C}>0$, if its entries are all positive. We say a non-negative matrix $U_{C}$ is stochastic if all rows add to $1, \sum_{j \in C} u(i, j)=1$ for all $i \in C$; of course, $U_{C}$ is substochastic when $\sum_{j \in C} u(i, j) \leq 1$ for all $i \in C$. Also, we say $U_{C}$ is primitive if there is an integer $k \geq 1$ such that $U_{C}^{k}>0$ is positive. In addition, we say $U_{C}$ is irreducible if for any $i, j \in C$, there is a finite path $i=x_{0}, x_{1}, \ldots, x_{n}=j$ in $C$ with positive weight, $U_{C}\left(x_{0}, x_{1}\right) \cdots U_{C}\left(x_{n-1}, x_{n}\right)>0$. The period of a state $i \in C$ is defined as $d_{C}(i)=$ g.c.d $\left\{n \geq 1: U_{C}^{n}(i, i)>0\right\}$. When $U_{C}$ is irreducible, all states in $C$ have the same period $d_{C}$. When $d_{C}=1$, we say $U_{C}$ is aperiodic. Finally, note that $U_{C}$ is primitive $\Leftrightarrow U_{C}$ is irreducible and aperiodic $\Leftrightarrow\left(U_{C}\right)^{\mathfrak{r}}>0$. 
Construction (CON). We now construct a sequence of non-negative "Markov"-like measures. Let $U_{k}=\left\{u_{k}(i, j)\right\}$ for $1 \leq k \leq n$ be a sequence of of $\mathfrak{r} \times \mathfrak{r}$ non-negative matrices. Let also $\pi$ be a measure on $\Sigma$. Then, define the non-negative measure $\mathbb{U}_{\pi}$ on $\Sigma^{n}$ for $n \geq 1$ where $\mathbb{U}_{\pi}\left(X_{0} \in B\right)=\pi(B)$, and

$$
\mathbb{U}_{\pi}\left(\vec{X}_{n} \in B\right)=\sum_{x_{0} \in \Sigma} \sum_{\vec{x}_{n} \in B} \pi\left(x_{0}\right) \prod_{i=1}^{n} u_{i}\left(x_{i-1}, x_{i}\right)
$$

where $\vec{X}_{n}=\left\langle X_{1}, \ldots, X_{n}\right\rangle$ is the coordinate process up to time $n$. Let also $\vec{X}_{i}^{j}=$ $\left\langle X_{i}, \ldots, X_{j}\right\rangle$, for $0 \leq i \leq j$, be the observations between times $i$ and $j$ and denote, for $0 \leq k \leq m \leq l$,

$$
\mathbb{U}_{(k, \pi)}\left(\vec{X}_{m}^{l} \in B\right)=\mathbb{U}_{\pi}^{\prime}\left(\vec{X}_{m-k}^{l-k} \in B\right)
$$

where $\mathbb{U}_{\pi}^{\prime}$ is made with respect to $U_{i}^{\prime}=U_{i+k}$ for $i \geq 1$. When $\pi$ is the point mass $\delta_{x}$ for $x \in \Sigma$, we denote $\mathbb{U}_{\left(k, \delta_{x}\right)}=\mathbb{U}_{(k, x)}$ for simplicity.

The measure $\mathbb{U}_{\pi}$ shares the "Markov" property:

$$
\begin{aligned}
\mathbb{U}_{\pi}\left(\vec{X}_{k} \in A, \vec{X}_{k+1}^{n} \in B\right) & =\sum_{x_{0} \in \Sigma} \sum_{\vec{x}_{k} \in A} \sum_{\vec{x}_{k+1}^{n} \in B} \pi\left(x_{0}\right) \prod_{i=1}^{n} u_{i}\left(x_{i-1}, x_{i}\right) \\
& =\sum_{\vec{x}_{k} \in A} \mathbb{U}_{\pi}\left(\vec{X}_{k}=\vec{x}_{k}\right) \mathbb{U}_{\left(k, x_{k}\right)}\left(\vec{X}_{k+1}^{n} \in B\right) .
\end{aligned}
$$

LDP for Homogeneous Non-negative Processes. Let $U$ be a non-negative matrix on $\Sigma$. Let also $C \subset \Sigma$ and $f: \Sigma \rightarrow \mathbb{R}^{d}$ be a subset and function on the state space.

For $\lambda \in \mathbb{R}^{d}$, define the "tilted" matrix $\Pi_{C, \lambda, f, U}=\Pi_{C, \lambda}$ by

$$
\Pi_{C, \lambda}=\left\{u(i, j) e^{\langle\lambda, f(j)\rangle}: i, j \in C\right\} .
$$

Suppose now that $C$ is such that $U_{C}$ is irreducible. Then, $\Pi_{C, \lambda}$ is irreducible for all $\lambda$ and $f$, and we may define

$$
\rho(C, \lambda)=\rho(C, \lambda ; f, U) \text { as the Perron-Frobenius eigenvalue of } \Pi_{C, \lambda}
$$

(cf. Theorem 3.1.1 [10], or [29]). Define also the extended function $\mathbb{I}_{C}=\mathbb{I}_{C, f, U}: \mathbb{R}^{d} \rightarrow$ $\mathbb{R} \cup\{\infty\}$ by

$$
\mathbb{I}_{C, f, U}(x)=\sup _{\lambda \in \mathbb{R}^{d}}\{\langle\lambda, x\rangle-\log \rho(C, \lambda)\}
$$

and let $Q_{C}=Q_{\mathbb{I}_{C}}$ be its domain of finiteness.

Let now $\pi$ be a distribution on $\Sigma$, and let $\mathbb{U}_{\pi}$ be made from Construction (CON) with $U_{k}=U$ for all $k \geq 1$. We call such a measure $\mathbb{U}_{\pi}$ a homogeneous non-negative process. Also, for $x_{0} \in C$, define the measures on $\mathbb{R}^{d}$, for $n \geq 2$ by

$$
\mu_{n}(B)=\mathbb{U}_{x_{0}}\left(Z_{n}(f) \in B, \vec{X}_{n} \in C^{n}\right) .
$$


Define also for $1 \leq k \leq l$ that $Z_{k}^{l}=Z_{k}^{l}(f)=(1 / l-k+1) \sum_{i=k}^{l} f\left(X_{i}\right)$. Note, as $|\Sigma|<\infty$, that $f$ is bounded, $\|f\|=\max _{1 \leq i \leq d}\left\|f_{i}\right\|_{L^{\infty}}<\infty$, and so $Z_{k}^{l}$ varies within the closed cube $\mathbb{K}=\bar{B}_{c u}(0,\|f\|)$ of width $2\|f\|$ about the origin.

The following is proved in the appendix.

Proposition 2.1 The function $\mathbb{I}_{C}$ and domain $Q_{C}$ satisfy the following.

1. $Q_{C}$ is a non-empty convex compact subset of the cube $\mathbb{K}$.

2. $\mathbb{I}_{C}$ is a good extended rate function. In fact, when $U_{C}$ is substochastic, $\mathbb{I}_{C}$ is a good rate function.

3. $\mathbb{I}_{C}$ is convex on $\mathbb{R}^{d}$ and strictly convex on the relative interior of $Q_{C}$. Also, when restricted to $Q_{C}, \mathbb{I}_{C}$ is uniformly continuous, and hence bounded on $Q_{C}$.

4. $\left\{\mu_{n}\right\}$ satisfies an LDP (2.1) with extended rate function $\mathbb{I}_{C}$.

Upper Block Form. For a stochastic matrix $P=\{p(i, j)\}$ on $\Sigma$, we now recall the "upper block" form. By reordering $\Sigma$ if necessary, the matrix $P$ may be put in form

$$
P=\left[\begin{array}{ccccc}
U(0,0) & U(0,1) & \ldots \ldots & U\left(0, M_{0}\right) \\
0 & S(1) & 0 & \ldots & 0 \\
\vdots & 0 & \ddots & & \vdots \\
\vdots & & \ddots & \ddots & \vdots \\
0 & \ldots \ldots \ldots \ldots & 0 & S\left(M_{0}\right)
\end{array}\right]
$$

where $1 \leq M_{0} \leq \mathfrak{r}$, and $S(1), \ldots, S\left(M_{0}\right)$ are stochastic irreducible submatrices corresponding to disjoint subsets of recurrent states-denoted as stochastic sets-and submatrices $U(0,0), \ldots, U\left(0, M_{0}\right)$ correspond to transient states when they exist.

When there are transient states, the square block $U(0,0)$ itself may be decomposed as (cf. [29], section 1.2)

$$
U(0,0)=\left[\begin{array}{ccccc}
R(1) & V(1,2) & \ldots \ldots \ldots & V\left(1, N_{0}\right) \\
0 & R(2) & V(2,3) & \ldots & V\left(2, N_{0}\right) \\
\vdots & 0 & \ddots & & \vdots \\
\vdots & & \ddots & \ddots & \vdots \\
0 & \ldots \ldots & \ldots & 0 & R\left(N_{0}\right)
\end{array}\right]
$$

where $1 \leq N_{0} \leq \mathfrak{r}-1$, and $R(i)$ is either the $1 \times 1$ zero matrix or an irreducible submatrix corresponding to a subset of transient states for $1 \leq i \leq N_{0}$. We will call the $R(i)=[0]$ matrices and corresponding states as degenerate transient, and the irreducible $R(i)$ and associated states as nondegenerate transient since respectively returns to these states are impossible, and possible under the time-homogeneous chain run with $P$.

Define now $N$ as the number of degenerate transient submatrices,

$$
N=\left\{\begin{aligned}
0 & \text { when no transient states in } P \\
\left|\left\{1 \leq i \leq N_{0}: R(i)=[0]\right\}\right| & \text { otherwise. }
\end{aligned}\right.
$$


Let also $M$ be the number of nondegenerate and stochastic submatrices,

$$
M=\left\{\begin{aligned}
M_{0} & \text { when no transient states in } P \\
\left(N_{0}-N\right)+M_{0} & \text { otherwise. }
\end{aligned}\right.
$$

It will be useful to rewrite the upper block form by inserting the form for $U(0,0)$ into (2.4). To this end, when there are transient states, let $P(i)=R(i)$ for $1 \leq i \leq N_{0}$, and $P(i)=S\left(i-N_{0}\right)$ for $N_{0}+1 \leq i \leq N_{0}+M_{0}$. When all states are recurrent, let $P(i)=S(i)$ for $1 \leq i \leq M_{0}$. Also, in the following, let $T(i, j)$ for $i<j$ denote the appropriate "connecting" submatrix $U(\cdot, \cdot)$ or $V(\cdot, \cdot)$. We remark that $T(i, j)$ is a matrix of zeroes for $N_{0}+1 \leq i<j \leq N+M$.

We have now the canonical decomposition of $P$,

$$
P=\left[\begin{array}{ccccc}
P(1) & T(1,2) & \ldots \ldots \ldots & T(1, N+M) \\
0 & P(2) & T(2,3) & \cdots & T(2, N+M) \\
\vdots & 0 & \ddots & & \vdots \\
\vdots & & \ddots & \ddots & \vdots \\
0 & \ldots \ldots \ldots \ldots & 0 & P(N+M)
\end{array}\right]
$$

Let now $C_{i}=C_{i}(P) \subset \Sigma$ be the subset which corresponds to $P(i)$ so that $P_{C_{i}}=$ $P(i)=\left\{p(x, y): x, y \in C_{i}\right\}$ for $1 \leq i \leq N+M$. Define also the sets $\mathcal{D}=\mathcal{D}(P)$, $\mathcal{N}=\mathcal{N}(P), \mathcal{M}=\mathcal{M}(P)$ and $\mathcal{G}=\mathcal{G}(P)$ by

$$
\begin{aligned}
\mathcal{D} & =\{i: P(i) \text { degenerate transient }\} \\
\mathcal{N} & =\{i: P(i) \text { nondegenerate transient }\} \\
\mathcal{M} & =\{i: P(i) \text { stochastic }\} \\
\mathcal{G} & =\mathcal{N} \cup \mathcal{M}(=\{i: P(i) \text { nondegenerate transient or stochastic }\}) .
\end{aligned}
$$

To link with previous notation, note that $N=|\mathcal{D}|, M=|\mathcal{G}|$.

It will be convenient to enumerate the elements of $\mathcal{G}$ as $\mathcal{G}=\left\{\zeta_{1}, \zeta_{2}, \ldots, \zeta_{M}\right\}$. As $P(i)$ is (sub)stochastic and irreducible for $i \in \mathcal{G}$, we may denote, with respect to $f: \Sigma \rightarrow \mathbb{R}^{d}$, the rate function $\mathbb{I}_{i}=\mathbb{I}_{C_{i}, f, P}$, and its domain of finiteness $Q_{i}=Q_{C_{i}}$. In addition, let

$$
p_{\text {min }}=\min \left\{p(x, y): p(x, y) \neq 0, x, y \in C_{i}, i \in \mathcal{G}\right\}
$$

be the minimum positive transition probability in the irreducible submatrices of $P$.

Consider now a sequence of transition matrices $\left\{P_{n}\right\}$ where $P_{n}=\left\{p_{n}(i, j)\right\}$ converges to $P$. With respect to the sets $\left\{C_{i}(P): 1 \leq i \leq N+M\right\}$ above for the matrix $P$, the $n$th step matrix $P_{n}$ can be put in form,

$$
P_{n}=\left[\begin{array}{ccccccc}
P_{n}(1) & T_{n}(1,2) & \ldots \ldots \ldots \ldots \ldots \ldots \ldots \ldots \ldots & T_{n}(1, N+M) \\
T_{n}(2,1) & P_{n}(2) & T_{n}(2,3) & \ldots & T_{n}(2, N+M) \\
\vdots & T_{n}(3,2) & & & \vdots \\
\vdots & & \ddots & & \vdots \\
T_{n}(N+M, 1) & \ldots \ldots \ldots \ldots \ldots & T_{n}(N+M, N+M-1) & P_{n}(N+M)
\end{array}\right]
$$


where $P_{n}(i)=\left(P_{n}\right)_{C_{i}} \rightarrow P(i)$ for $1 \leq i \leq N+M$, and $T_{n}(i, j)$ governs $P_{n}$-transitions from $C_{i}$ to $C_{j}$ and $T_{n}(i, j) \rightarrow T(i, j)$ for $i<j$ and vanishes otherwise. As a warning, we note the form above for $P_{n}$ is NOT the canonical decomposition of $P_{n}$.

Routing Costs and Deviations. Let $\mathbb{S}_{M}$ and $\Omega_{M}$ be the set of permutations, and collection of probability vectors on $\{1,2, \ldots, M\}$,

$$
\Omega_{M}=\left\{\vec{v} \in \mathbb{R}^{M}: \sum_{i=1}^{M} v_{i}=1, \quad 0 \leq v_{i} \leq 1 \text { for } 1 \leq i \leq M\right\} .
$$

For $\vec{v} \in \Omega_{M}$ and $z \in \mathbb{R}^{d}$, define the set of convex combinations,

$$
D(M, \vec{v}, z)=\left\{\vec{x}=\left\langle x_{1}, \ldots, x_{M}\right\rangle \in\left(\mathbb{R}^{d}\right)^{M}: \sum_{i=1}^{M} v_{i} x_{i}=z\right\}
$$

Let also $U=\{u(i, j): 1 \leq i, j \leq M\}$ be a matrix of extended non-positive real numbers. For a permutation $\sigma \in \mathbb{S}_{M}, \vec{v} \in \Omega_{M}, \vec{x} \in\left(\mathbb{R}^{d}\right)^{M}$, and $z \in \mathbb{R}^{d}$, define the extended functions

$$
C_{\vec{v}, U}(\sigma, \vec{x})=\left\{\begin{array}{rr}
-\sum_{i=1}^{M-1}\left(\sum_{j=1}^{i} v_{j}\right) u\left(\zeta_{\sigma(i)}, \zeta_{\sigma(i+1)}\right)+\sum_{i=1}^{M} v_{i} \mathbb{I}_{\zeta_{\sigma(i)}}\left(x_{i}\right) & \text { when } M \geq 2 \\
\mathbb{I}_{1}\left(x_{1}\right) & \text { when } M=1
\end{array}\right.
$$

and

$$
\mathbb{J}_{U}(z)=\inf _{\vec{v} \in \Omega_{M}} \inf _{\vec{x} \in D(M, \vec{v}, z)} \min _{\sigma \in S_{M}} C_{\vec{v}, U}(\sigma, \vec{x}) .
$$

It will be shown that $\mathbb{J}_{U}$ is a good rate function (Proposition 4.1). Moreover, it will turn out, for well chosen "routing cost" matrices $U$, that $\mathbb{J}_{U}(z)$ measures various upper and lower large deviation rates of the additive sums $\left\{Z_{n}(f)\right\}$. Note that $\mathbb{J}_{U}$ is defined in terms of $\left\{\zeta_{i}\right\}=\mathcal{G}$ and depends on $i \in \mathcal{D}$ only possibly through the "routing" cost $U$ which makes sense since it would be too expensive to "rest" on degenerate transient states in any positive time proportion. Also, we observe when $M=1$, that is when any transient states with respect to $P$ do not allow returns, and $P$ corresponds to exactly one irreducible stochastic block, the function $\mathbb{J}_{U}(z)=\mathbb{I}_{1}(z)$ is independent of $U$.

Upper and Lower Cost Matrices. With respect to a $\mathbb{P}_{\pi} \in \mathbb{A}(P)$, we now specify certain relevant "upper" and "lower" $\operatorname{costs} U$ when $N+M \geq 2$. Define, for distinct $1 \leq i, j \leq N+M$

$$
t(n,(i, j))=\max _{x \in C_{i}, y \in C_{j}} p_{n}(x, y)
$$

and the extended non-positive numbers

$$
v(i, j)=\varlimsup_{n \rightarrow \infty} \frac{1}{n} \log t(n,(i, j)) \quad \text { and } \quad \tau(i, j)=\varliminf_{n \rightarrow \infty} \frac{1}{n} \log t(n,(i, j)) .
$$


Also, for $0 \leq k \leq N+M-2$, let $l_{0}=i, l_{k+1}=j$ and $L_{k}=\left\langle l_{0}, l_{1}, \ldots, l_{k}, l_{k+1}\right\rangle$ be a $k+2$-tuple of distinct indices. Now define the "upper" cost

$$
\mathcal{U}_{0}(i, j)=\max _{0 \leq k \leq N+M-2} \max _{L_{k}} \sum_{s=0}^{k} v\left(l_{s}, l_{s+1}\right)
$$

and "lower cost"

$$
\mathcal{T}_{0}(i, j)=\max _{0 \leq k \leq N+M-2} \max _{L_{k}} \sum_{s=0}^{k} \tau\left(l_{s}, l_{s+1}\right) .
$$

We remark briefly that $\mathcal{U}_{0}(i, j)$ and $T_{0}(i, j)$ represent respectively "maximal" and "minimal" asymptotic travel costs of moving from $C_{i}$ to $C_{j}$ in $k+1 \leq N+M-1$ steps by visiting sets $\left\{C_{i}\right\}$ in the order $L_{k}$.

A more subtle "lower" cost $\mathcal{T}_{1}$ is the following. Let $0 \leq k \leq N+M-2, l_{0}=i$, $l_{k+1}=j$ and $L_{k}$ be as before. Let also

$$
1 \leq q_{0}, q_{k+1} \leq \mathfrak{r} \text {, and when } k>1 \text { and } 1 \leq s \leq k \text { let } 1 \leq q_{s} \leq \mathfrak{r}+1
$$

and call $Q_{k}=\left\langle q_{0}, \ldots, q_{k+1}\right\rangle$. Let $\vec{x}^{0}=\left\langle x_{1}^{0}, \ldots, x_{q_{0}}^{0}\right\rangle$ and $\vec{x}^{k+1}=\left\langle x_{1}^{k+1}, \ldots, x_{q_{k+1}}^{k+1}\right\rangle$ be vectors with components in $C_{i}$ and $C_{j}$ respectively, and when $k \geq 1$ let $\vec{x}^{i}=$ $\left\langle x_{1}^{i}, \ldots, x_{q_{i}}^{i}\right\rangle$ be a vector with elements in $C_{l_{i}}$ for $1 \leq i \leq k$. Denote also the $k+2$-tuple $V_{k}=\left\langle\vec{x}^{0}, \vec{x}^{1}, \ldots, \vec{x}^{k+1}\right\rangle$.

For distinct $i, j \in \mathcal{G}$, and $y \in C_{i}$ and $z \in C_{j}$, define

$$
\underline{\gamma}^{1}(n, y, z)=\max _{0 \leq k \leq N+M-2} \max _{L_{k}} \max _{Q_{k}} \max _{V_{k}} \mathbb{P}_{(n-1, y)}\left(\vec{X}_{n}^{n+r(k+1)}=\left\langle\vec{x}^{0}, \ldots, \vec{x}^{k+1}, z\right\rangle\right)
$$

where the "concatenated" vector $\left\langle\vec{x}^{0}, \ldots, \vec{x}^{k+1}, z\right\rangle=\left\langle x_{1}^{0}, \ldots, x_{q_{k+1}}^{k+1}, z\right\rangle$ is of length at most $E_{0}(N, M)+1$. Here, $E_{0}(N, M)=(\mathfrak{r}+1)(M-2)+N+2 \mathfrak{r}$ and $r(u)=\sum_{l=0}^{u} q_{l}$ for $0 \leq u \leq k+1$.

Also, define that

$$
\underline{\gamma}^{1}(n,(i, j))=\inf _{y \in C_{i}, z \in C_{j}} \underline{\gamma}^{1}(n, y, z)
$$

Finally, define

$$
\mathcal{T}_{1}(i, j)=\underline{\lim } \frac{1}{n} \log \underline{\gamma}^{1}(n,(i, j)) .
$$

We now interpret the objects $\underline{\gamma}^{1}(n, y, z), \underline{\gamma}^{1}(n,(i, j))$, and $\mathcal{T}_{1}(i, j)$. As with the "routing" cost $\mathcal{T}_{0}, L_{k}$ is an ordered list of sets to visit on the way from point $y$ to $z$. But more specifically here, $Q_{k}$ lists the $O(\mathfrak{r})$ number of steps taken in each visited set, and $V_{k}$ details on which states this travel is made. Here, " $\mathfrak{r}$ " is chosen since all movement in a given irreducible $C_{i} \subset \Sigma$ is possible in at most $\mathfrak{r}=|\Sigma|$ steps. Then, $\gamma^{1}(n, y, z)$ is the largest probability of movement from $y$ to $z$ within the constraints of $O(\mathfrak{r})$ travel among distinct sets. Also, $\gamma^{1}(n,(i, j))$ is the smallest such chance of moving from $C_{i}$ to $C_{j}$, and $\mathcal{T}_{1}(i, j)$ is the asymptotic exponential rate of this quantity. 


\section{Results}

We now come to the main results for processes $\mathbb{P}_{\pi} \in \mathbb{A}(P)$. After a general upper bound, and some lower bounds which depend on natural assumptions, we present an LDP which follows from these bounds. Some remarks to the Metropolis scheme, and on the format of the article are made at the end of the section.

The upper bound statement is the following.

Theorem 3.1 With respect to good rate function $\mathbb{J}_{\mathcal{U}_{0}}$ and Borel $\Gamma \subset \mathbb{R}^{d}$, we have

$$
\varlimsup_{n \rightarrow \infty} \frac{1}{n} \log \mathbb{P}_{\pi}\left(Z_{n} \in \bar{\Gamma}\right) \leq-\inf _{z \in \bar{\Gamma}} \mathbb{J}_{\mathcal{U}_{0}}(z) .
$$

We now label conditions and assumptions to give LD lower bounds.

Sufficient Initial Ergodicity. To avoid "degenerate" cases, we introduce an "initial ergodicity" condition for $\mathbb{P}_{\pi}$ so that all information about $P$ is relevant. A typical situation to avoid is when $P_{n}=P$ for $n \geq m$, and distribution $\pi P_{1} \cdots P_{m}$ locks the process evolution into a strict $P$-irreducible subset of $\Sigma$. To avoid lengthy technicalities and to be concrete, we impose the following assumption on the chains considered in the article. Let $n_{0}=n_{0}\left(\left\{P_{n}\right\}\right) \geq 1$ be the first index $m$ so that for all $s, t \in C_{i}$ and $i \in \mathcal{G}$ when $p(s, t)>0$ we have $p_{n}(s, t)>0$ for $n \geq m$. Such an $n_{0}<\infty$ exists since $P_{n} \rightarrow P$.

Condition (SIE). There is an $n_{1} \geq n_{0}-1$ such that

$$
\mathbb{P}_{\pi}\left(X_{n_{1}} \in C_{i}\right)>0 \text { for all } i \in \mathcal{G} .
$$

A simpler condition which implies (SIE) is the following.

Condition (SIE-1). Let $n_{0}=1$, and let $\pi\left(C_{i}\right)>0$ for all $i \in \mathcal{G}$.

We say that a distribution $\pi$ is (SIE-1)-positive if $\pi\left(C_{i}\right)>0$ for all $i \in \mathcal{G}$. A trivial condition for (SIE-1)-positivity is when $\pi$ is positive, e.g. when $\pi(x)>0$ for all $x \in \Sigma$.

Assumptions $A, B$ and $C$. We now state three assumptions on the regularity of the asymptotic approach $P_{n} \rightarrow P$.

Assumption A. Suppose $v(i, j)=\tau(i, j)$ for all distinct $1 \leq i, j \leq N+M$.

Assumption B. Suppose for all distinct $1 \leq i, j \leq N+M$ there exists an element $a=a(i, j) \in C_{i}$ and a sequence $\left\{b_{n}=b_{n}(i, j)\right\} \subset C_{j}$ such that

$$
\tau(i, j)=\lim _{n \rightarrow \infty} \frac{1}{n} \log p_{n}\left(a, b_{n}\right) .
$$

In other words, $\tau(i, j)$ is achieved on a fixed departing point $a \in C_{i}$.

Assumption C. Define $P^{*}(i)=\left\{p^{*}(s, t): s, t \in C_{i}\right\}$ by

$$
p^{*}(s, t)=\left\{\begin{aligned}
p(s, t) & \text { when } p(s, t)>0 \\
1 & \text { when } \underline{\lim }(1 / n) \log p_{n}(s, t)=0 \text { and } p(s, t)=0 \\
0 & \text { otherwise }
\end{aligned}\right.
$$


Suppose that $P^{*}(i)$ is primitive for $i \in \mathcal{G}$.

In words, Assumption A specifies that the maximal "connection" probabilities in the " $(1 / n) \log$ " sense have limits. Assumption B states that $\tau(i, j)$ can be achieved in a systematic manner. Assumption $\mathrm{C}$ ensures there is "primitivity" in the system, and covers the case when $P$ is periodic but the approach $P_{n}$ is slow enough to give a sense of primitivity. We now list some easy sufficient conditions to verify these assumptions.

Proposition 3.1 (LIM): Assumption $A$ and $B$ hold if for distinct $1 \leq i, j \leq N+M$, and each pair $x \in C_{i}$ and $y \in C_{j}$, we have $\lim _{n \rightarrow \infty}(1 / n) \log p_{n}(x, y)$ exists.

(PRM): Assumption $C$ holds when $\{P(i): i \in \mathcal{G}\}$ are primitive.

We now come to lower bound statements for process obeying condition (SIE), the first of which holds in general, and the second under Assumption $B$ or $C$.

Theorem 3.2 Let $\mathbb{P}_{\pi}$ satisfy condition (SIE). Then, with respect to good rate function $\mathbb{J}_{\mathcal{T}_{1}}$ and Borel $\Gamma \subset \mathbb{R}^{d}$, we have

$$
\text { (i) } \quad-\inf _{z \in \Gamma^{o}} \mathbb{J}_{\mathcal{T}_{1}}(z) \leq \underline{\underline{\lim }} \frac{1}{n \rightarrow \infty} \log \mathbb{P}_{\pi}\left(Z_{n} \in \Gamma^{o}\right) .
$$

Also, when in addition either Assumption $B$ or $C$ holds, we have with respect to good rate function $\mathbb{J}_{\mathcal{T}_{0}}$ that

$$
\text { (ii) }-\inf _{z \in \Gamma^{o}} \mathbb{J}_{\mathcal{T}_{0}}(z) \leq \underline{\underline{\lim }} \frac{1}{n \rightarrow \infty} \log \mathbb{P}_{\pi}\left(Z_{n} \in \Gamma^{o}\right) .
$$

We note, in the case $M=1$, that is when $P$ possesses exactly one irreducible recurrent stochastic set and possibly some degenerate transient states, Theorems 3.1 and 3.2 already give an LDP with rate function $\mathbb{J}_{\mathcal{T}_{1}}=\mathbb{J}_{\mathcal{U}_{0}}=\mathbb{I}_{1}$. In particular, in this case, the large deviation behavior under $\mathbb{P}_{\pi}$ is independent of the approach $P_{n} \rightarrow P$.

However, in the general situation, when $M \geq 2$, the lower and upper bounds may be different. In fact, there are nonhomogeneous processes $\mathbb{P}_{\pi}$ for which the lower and upper rate function bounds in Theorems 3.1 and 3.2 (i) differ and are achieved so that the result is sharp in a certain sense, for instance the example in subsection 12.2.

Also, we remark that the two lower bounds in Theorem 3.2 may differ when there is some "periodicity" in the system and the maximal connection weight sequence is not regular. In this case, the process may not be allowed to visit freely various states as certain cyclic patterns may be in force. Therefore, the asymptotic routing costs in this general case should be larger than under Assumption B or $\mathrm{C}$ when some regularity is imposed on connection probabilities or when a form of primitivity is present. Hence, the use of $\mathcal{T}_{1}$ instead of $\mathcal{T}_{0}$ in the lower estimates. See subsection 12.3 for an explicit process where lower bounds do not respect $\mathcal{T}_{0}$.

It is natural now to ask when the lower and upper bounds match in the previous results so that a large deviation principle holds. For $z \in \mathbb{R}^{d}$, let

$$
\mathbb{J}(z)=\mathbb{J}_{\mathcal{U}_{0}}(z) .
$$


Under Assumption A, costs $\mathcal{T}_{0}=\mathcal{U}_{0}$, and so the following is a direct corollary of Thereoms 3.1 and 3.2 .

Theorem 3.3 Suppose $\mathbb{P}_{\pi}$ satisfies condition (SIE) and Assumption A, and also either Assumption $B$ or $C$. Then, with respect to good rate function $\mathbb{I}$ and Borel sets $\Gamma \subset \mathbb{R}^{d}$, we have the $L D P$,

$$
\begin{aligned}
-\inf _{z \in \Gamma^{o}} \mathbb{J}(z) & \leq \varliminf_{n \rightarrow \infty} \frac{1}{n} \log \mathbb{P}_{\pi}\left(Z_{n} \in \Gamma^{o}\right) \\
& \leq \varlimsup_{n \rightarrow \infty} \frac{1}{n} \log \mathbb{P}_{\pi}\left(Z_{n} \in \bar{\Gamma}\right) \leq-\inf _{z \in \bar{\Gamma}} \mathbb{J}(z) .
\end{aligned}
$$

Hence, by Proposition 3.1, when all limits exist (LIM) (in particular for instance in the time-homogeneous case, $P_{n} \equiv P$ ), or when Assumption A holds and there is no periodicity (PRM), the LDP is available. Also, note that by taking $f(x)=$ $\left\langle 1_{1}(x), 1_{2}(x), \ldots, 1_{\mathfrak{r}}(x)\right\rangle$, Theorem 3.3 gives the LDP for the empirical measure and so is a form of Sanov's theorem for these nonhomogeneous chains.

We remark that it may be tempting to think Assumption A by itself may be sufficient for an LDP, but it turns out there are processes which satisfy (SIE) and Assumption A but neither B nor C for which the LDP cannot hold, for instance the example in subsection 12.3. On the other hand, we note that Assumption A is not even necessary for an LDP-for instance with respect to chains where $P_{n}$ alternates between two alternatives (cf. subsection 12.1). So, although Theorem 3.3 is broad in a sense, more work is required to identify necessary and sufficient conditions for an LDP.

We now comment on the three types of LD behaviors mentioned in the introduction which follow from Theorem 3.3. These are (1) homogeneous, (2) trivial and (3) intermediate behaviors for which easy sufficient (but not necessary) conditions are below.

Corollary 3.1 Let Condition (SIE), and Assumption $A$ and either Assumption B or $C$ hold. Let also $N+M \geq 2$.

(1) Suppose $v(i, j)=-\infty$ when $\varlimsup t(n,(i, j))=0$ for distinct $1 \leq i, j \leq N+M$. Then, $\mathbb{J}$ is also the rate function for the time-homogeneous chain run under $P$ (as the "routing" costs are the same as if $P_{n} \equiv P$ )

(2) Suppose $|\mathcal{M}| \geq 2$, and $\mathcal{U}_{0}(i, j)=0$ for all distinct $i, j \in \mathcal{M}$. Then, $\mathbb{J}$ vanishes on the convex hull of $\cup_{i \in \mathcal{M}}\left\{z: \mathbb{I}_{i}(z)=0\right\}$, and so is in a sense "trivial."

(3) Suppose $|\mathcal{M}| \geq 2$, and $\mathcal{U}_{0}(i, j) \in(-\infty, 0)$ for all distinct $i, j \in \mathcal{M}$. Then, $\mathbb{J}$ differs from the rate function for the time-homogeneous chain run with $P$, and also involves nontrivially the convergence speed of $P_{n}$ to $P$ in terms of "routing" costs.

We now briefly comment on application to the Metropolis algorithm. Note

$$
1-\sum_{j \neq i} p_{n}(i, j)=g(i, i)+\sum_{j \neq i} g(i, j)\left[1-e^{-\beta_{n}(H(j)-H(i))_{+}}\right] .
$$


Also, as $\beta_{n} \rightarrow \infty$, we have $\lim _{n \rightarrow \infty} g(i, j) \exp \left\{-\beta_{n}(H(j)-H(i))_{+}\right\}=g(i, j) 1_{[H(j) \leq H(i)]}$. Therefore, the limit matrix $P$ is formed in terms of entries

$$
\lim _{n} p_{n}(i, j)=\left\{\begin{aligned}
g(i, j) 1_{[H(j) \leq H(i)]} & \text { if } i \neq j \\
g(i, i)+\sum_{j \neq i} g(i, j) 1_{[H(j)>H(i)]} & \text { if } i=j
\end{aligned}\right.
$$

We now decompose $P$ into components $\mathcal{D}, \mathcal{N}$ and $\mathcal{M}$. First, note that a state $x \in \Sigma$ belongs to the "level" set

$$
\begin{gathered}
C_{x}=\{x\} \cup\left\{y: \exists \text { path } x=x_{0}, \ldots, x_{n}=y, \text { where } \prod_{i=0}^{n-1} g\left(x_{i}, x_{i+1}\right)>0\right. \\
\text { and } \left.H\left(x_{i}\right)=H(x) \text { for } 1 \leq i \leq n\right\}
\end{gathered}
$$

which corresponds to one of three types, $\mathcal{D}, \mathcal{N}$ or $\mathcal{M}$.

In particular, $C_{x}$ is a stochastic set corresponding to $\mathcal{M}$ exactly when $H(x)=$ $\min \{H(y): g(x, y)>0\}$ is a local minimum. Also, $C_{x}$ is a non-degenerate transient set exactly when $H(x)$ is not a local minimum and either $g(x, x)>0$ or $g(x, y)>0$ where $H(y)=H(x)$. And, $C_{x}$ is a degenerate singleton exactly when $H(x)$ is not a local minimum, $g(x, x)=0$, and when $g(x, y)>0$ we have $H(y) \neq H(x)$.

We now discuss the rate of convergence $P_{n} \rightarrow P$. Observe for distinct $1 \leq i, j \leq$ $N+M$ and $x \in C_{i}$ and $y \in C_{j}$ that

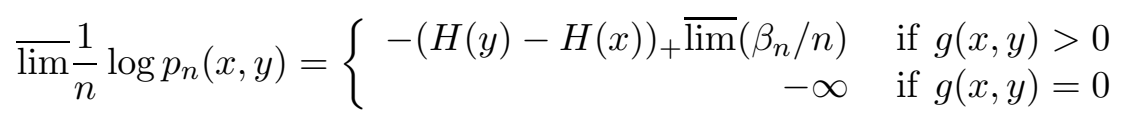

with analogous expressions for $\left.\underline{\lim }(1 / n) \log p_{n}(x, y)\right)$. Hence (LIM) holds when $\beta=$ $\lim \beta_{n} / n$ exists. Also, we remark, when $g(x, x)>0$ for $x \in \Sigma$, there are no degenerate transient states, and so all $P$-submatrices are primitive and (PRM) holds. In addition, given irreducibility of $g$, (SIE) is satisfied with respect to any initial distribution $\pi$.

Therefore, by Corollary 3.1, as routing costs are computed with respect to different level sets, the three types of LD behavior follow when the limit $\beta$ exists and there is more than one local minimum. Namely, trivial, intermediate or homogeneous behaviors occur when $\beta=0, \beta \in(0, \infty)$, or $\beta=\infty$.

Finally, we give a concrete example with respect to a simple geometrically cooling Metropolis chain where $\beta=1$. Let $H$ be defined on $\Sigma=\{1,2, \ldots, 9\}$ in terms of its graph (Fig. 1), and let $f(x)=H(x)$, so that $Z_{n}$ is the average $H$-value seen by the chain. Typically, for large $n$, these values $Z_{n}$ will be near an $H$-local minimum average. 


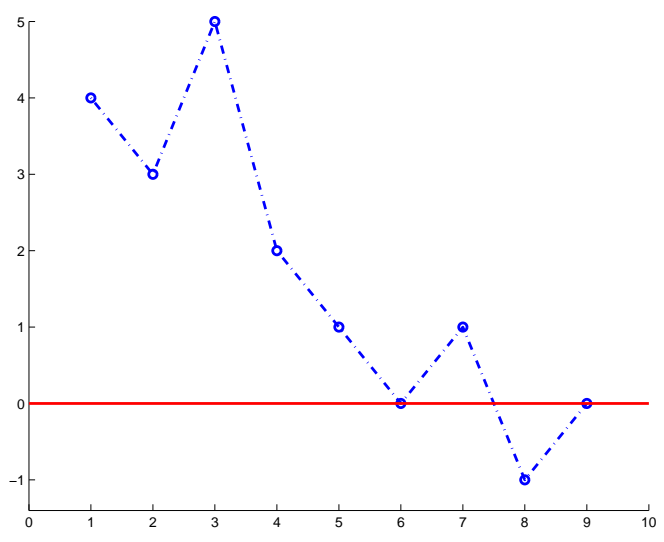

Fig. 1: Graph of $H$

Let the kernel $g$ be a random walk so that $g(i, i+1)=1 / 2$ for $i=2,6,7,8$, $g(i+1, i)=1 / 2$ for $i=1,2,6,7$, and $g(1,2)=1, g(9,8)=1, g(3,4)=1 / 2, g(4,3)=$ $(1-a) / 2, g(4,4)=a, g(4,5)=(1-a) / 2, g(5,4)=(1-b) / 2, g(5,5)=b$, and $g(5,6)=(1-b) / 2$ with $0<a, b<1$. Then, states $\{2\},\{6\},\{8\}$ are distinct local minima, $\{4\},\{5\}$ are nondegenerate transient singletons, and the remaining states are degenerate transient.

The routing costs satisfy, for distinct sets,

$$
\mathcal{U}_{0}(\{i\},\{j\})= \begin{cases}-\sum_{l=i}^{j-1}(H(l)-H(l+1))_{+} & \text {for } i<j \\ -\sum_{l=j}^{i-1}(H(l+1)-H(l))_{+} & \text {for } i>j .\end{cases}
$$

Also, the rate functions corresponding to local minima 2,6, and 8 are degenerate and equal $\infty \cdot 1_{H(2)}(y), \infty \cdot 1_{H(6)}$, and $\infty \cdot 1_{H(8)}$ respectively. For the nondegenerate transient states 4 and 5 , we have

$$
I_{\{4\}}(y)=\left\{\begin{array}{rc}
-\log \frac{1+a}{2} & \text { for } y=H(4) \\
\infty & \text { otherwise }
\end{array} \quad \text { and } I_{\{5\}}(y)=\left\{\begin{array}{rc}
-\log \frac{1+b}{2} & \text { for } y=H(5) \\
\infty & \text { otherwise }
\end{array}\right.\right.
$$

When $-\log (1+a) / 2=1 / 3$ and $-\log (1+b) / 2=2 / 3$, we compute, by analyzing the not-too-large number of possibilities, the non-convex rate function

$$
\mathbb{J}(z)=\left\{\begin{aligned}
\infty & \text { for } z<-1 \text { and } z>3 \\
4 z / 9+4 / 9 & \text { for }-1 \leq z \leq-2 / 11 \\
-2 z & \text { for }-2 / 11 \leq z \leq 0 \\
z / 6 & \text { for } 0 \leq z \leq 2 \\
5 z / 3-3 & \text { for } 2 \leq z \leq 12 / 5 \\
-5 z / 3+5 & \text { for } 12 / 5 \leq z \leq 3
\end{aligned}\right.
$$




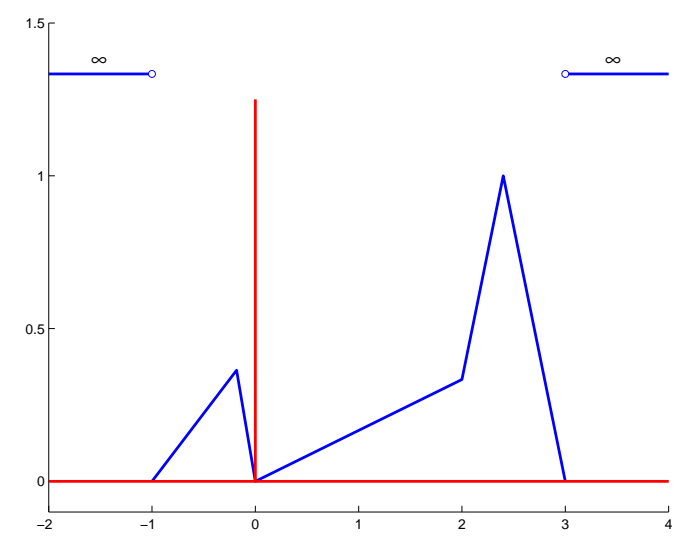

Fig. 2: Graph of J.

Not surprisingly, $\mathbb{J}$ vanishes at local minima, and is largest near $z \sim 2^{+}$(excluding infinite costs), with exact value $z=12 / 5$ found from computation. The $\mathbb{J}$-calculation also gives optimal scenarios under which $Z_{n} \sim z$; these include for $-1 \leq z \leq-2 / 11$ that the average $Z_{n}$ is a convex combination of "rest" stays initially on $\{4\}$ and then at $\{8\}$; for $-2 / 11 \leq z \leq 0$, at $\{8\}$, then $\{6\}$; for $0 \leq z \leq 2$, at $\{4\}$, then $\{6\}$; for $2 \leq z \leq 12 / 5$, at $\{2\}$, then $\{4\}$; for $12 / 5 \leq z \leq 3$, at $\{6\}$, then $\{2\}$.

We now discuss the plan of the paper. In the next section, we outline the proof structure of the main theorems. After supplying proofs of stated results in the outline in sections 5-11, we give three examples in section 12 remarked on earlier. Finally, in the appendix some technical proofs are collected.

\section{Outline of the Proofs of Main Theorems}

Consider a process $\mathbb{P}_{\pi} \in \mathbb{A}(P)$ and a function $f: \Sigma \rightarrow \mathbb{R}^{d}$. We first observe that $\mathbb{J}_{\mathcal{U}_{0}}$, $\mathbb{J}_{\mathcal{T}_{0}}$ and $\mathbb{J}_{\mathcal{T}_{1}}$ are all good rate functions from the following proposition proved in the appendix.

Proposition 4.1 For a nonpositive cost $U$, the function $\mathbb{J}_{U}$ is a good rate function, and the domain of finiteness $Q_{\mathbb{J}_{U}} \subset \mathbb{K}$.

In the following, we say that the path $\vec{X}_{n}$ enters or visits a subset $C \subset \Sigma$ when $X_{i} \in C$ for some $1 \leq i \leq n$. We now outline the proofs of Theorem 3.1 and 3.2.

\subsection{Upper Bounds: Proof of Theorem 3.1}

The proof follows by first a "surgery of paths" estimate, then a "homogeneous rest cost" comparison, a "coarse graining cost" estimate, and finally a limit relation on a perturbed rate function. Let $\Gamma \subset \mathbb{R}^{d}$ be a Borel set. 
Surgery of Paths Estimate. The first step is to overestimate $\mathbb{P}_{\pi}$ by another measure $\hat{\mu}_{\pi, \epsilon_{1}, \epsilon_{2}}$ which allows more movement in terms of parameters $\epsilon_{1}, \epsilon_{2}>0$. However, we will restrict the process to those paths which make at most one "long" sojourn to each of the sets $\left\{C_{i}: 1 \leq i \leq N+M\right\}$, but connect among them in "short" visits.

Before getting to the first bound, the following technical "monotonicity" lemma, proved in the appendix, is needed.

Lemma 4.1 Let $\delta \in[0,1]$, and $\left\{t_{n}\right\} \subset[0,1]$ be a sequence which converges to $\delta$. Then, there exists a sequence $\left\{\hat{t}_{n}\right\} \subset(0,1]$ such that (i) $t_{n} \leq \hat{t}_{n}$, (ii) $\hat{t}_{n} \downarrow \delta$ monotonically, and (iii) the limit $\lim (1 / n) \log \hat{t}_{n}$ exists and equals

$$
\lim _{n \rightarrow \infty} \frac{1}{n} \log \hat{t}_{n}=\varlimsup_{n \rightarrow \infty} \frac{1}{n} \log t_{n} .
$$

Recall now the definition of $t(n,(i, j))$ (cf. (2.7)), and let

$$
\{\hat{t}(n,(i, j))\} \text { be the sequence made from }\{t(n,(i, j))\} \text { and Lemma 4.1. }
$$

Also, for distinct $1 \leq i, j \leq N+M$, as in the definition of $\mathcal{U}_{0}(i, j)$ (cf. (2.8)), let $0 \leq k \leq N+M-2$, and let $l_{0}=i$ and $l_{k+1}=j$, and $L_{k}=\left\langle l_{0}, l_{1}, \ldots, l_{k}, l_{k+1}\right\rangle$ be composed of distinct indices. Then, define

$$
\gamma(n,(i, j))=\max _{0 \leq k \leq N+M-2} \max _{L_{k}} \prod_{s=0}^{k} \hat{t}\left(n+s,\left(l_{s}, l_{s+1}\right)\right) .
$$

The term $\gamma(n,(i, j))$ bounds the largest possible transition probability between sets $C_{i}$ and $C_{j}$ in at most $N+M-1$ steps.

We now create a certain sequence of positive transition matrices. For general $P$ and approaching sequence $\left\{P_{n}\right\}$, the submatrices $P(i)$ and $P_{n}(i)$ for $1 \leq i \leq N+M$ need not be positive. It will be helpful however to majorize them as follows. Let $\epsilon \geq 0$, and let $P(i, \epsilon)=\left\{p(s, t ; \epsilon): s, t \in C_{i}\right\}$ and $P_{n}(i, \epsilon)=\left\{p_{n}(s, t ; \epsilon): s, t \in C_{i}\right\}$ where

$$
p(s, t ; \epsilon)=\max \{p(s, t), \epsilon\} \text { and } p_{n}(s, t ; \epsilon)=\max \left\{p_{n}(s, t), \epsilon\right\} .
$$

Define now $\hat{\mathcal{P}}_{n, \epsilon_{1}, \epsilon_{2}}=\left\{\hat{p}_{n, \epsilon_{1}, \epsilon_{2}}(s, t)\right\}$ by

$$
\hat{p}_{n, \epsilon_{1}, \epsilon_{2}}(s, t)= \begin{cases}\gamma(n,(i, j)) & \text { for } s \in C_{i}, t \in C_{j}, \text { and distinct } 1 \leq i, j \leq N+M \\ p_{n}\left(s, t ; \epsilon_{2}\right) & \text { for } s, t \in C_{i}, \text { and } i \in \mathcal{G} \\ p_{n}\left(s, t ; \epsilon_{1}\right) & \text { for } s, t \in C_{i}, \text { and } i \in \mathcal{D}\end{cases}
$$

when $n \geq 2$, and for $n=1$, let $\hat{\mathcal{P}}_{1, \epsilon_{1}, \epsilon_{2}}$ be the unit constant matrix, $\hat{p}_{1, \epsilon_{1}, \epsilon_{2}}(s, t) \equiv 1$. Form also through Construction (CON) the measure $\hat{\mu}_{\pi, \epsilon_{1}, \epsilon_{2}}$ with respect to initial distribution $\pi$ and transition matrices $\left\{\hat{\mathcal{P}}_{n, \epsilon_{1}, \epsilon_{2}}\right\}$. 
Proposition 4.2 For $\epsilon_{1}, \epsilon_{2}>0$, the upper bound holds, $\varlimsup \frac{1}{n} \log \mathbb{P}_{\pi}\left(Z_{n} \in \Gamma\right) \leq \varlimsup \frac{1}{n} \log \hat{\mu}_{\pi, \epsilon_{1}, \epsilon_{2}}\left(Z_{n} \in \Gamma, \vec{X}_{n}\right.$ enters each $C_{i}$ at most once).

The proof of this proposition is found in section 5 .

Homogeneous Rest Cost Comparison. Next, we compare measure $\hat{\mu}_{\pi, \epsilon_{1}, \epsilon_{2}}$ with a measure $\bar{\mu}_{\pi, \epsilon_{1}, \epsilon_{2}}$ which replaces "nonhomogeneous" transitions within sets $C_{i}$ by "limiting homogeneous" transition weights.

Define, for $\epsilon_{1}, \epsilon_{2} \geq 0, \overline{\mathcal{P}}_{n, \epsilon_{1}, \epsilon_{2}}=\left\{\bar{p}_{n, \epsilon_{1}, \epsilon_{2}}(s, t)\right\}$ by

$$
\bar{p}_{n, \epsilon_{1}, \epsilon_{2}}(s, t)=\left\{\begin{aligned}
\gamma(n,(i, j)) & \text { for } s \in C_{i}, t \in C_{j}, \text { and distinct } 1 \leq i, j \leq N+M \\
p\left(s, t ; \epsilon_{2}\right) & \text { for } s, t \in C_{i}, \text { and } i \in \mathcal{G} \\
\epsilon_{1} & \text { for } s, t \in C_{i}, \text { and } i \in \mathcal{D}
\end{aligned}\right.
$$

when $n \geq 2$, and $\overline{\mathcal{P}}_{1, \epsilon_{1}, \epsilon_{2}}=\hat{\mathcal{P}}_{1, \epsilon_{1}, \epsilon_{2}}$. Let now $\bar{\mu}_{\pi, \epsilon_{1}, \epsilon_{2}}$ be formed from Construction $(\mathrm{CON})$ and matrices $\left\{\overline{\mathcal{P}}_{n, \epsilon_{1}, \epsilon_{2}}\right\}$ and $\pi$.

Proposition 4.3 For $\epsilon_{1}, \epsilon_{2}>0$, we have

$$
\begin{aligned}
& \varlimsup \frac{1}{n} \log \hat{\mu}_{\pi, \epsilon_{1}, \epsilon_{2}}\left(Z_{n} \in \Gamma, \vec{X}_{n} \text { enters each } C_{i}\right. \text { at most once) } \\
& \quad \leq \varlimsup \frac{1}{n} \log \bar{\mu}_{\pi, \epsilon_{1}, \epsilon_{2}}\left(Z_{n} \in \Gamma, \vec{X}_{n} \text { enters each } C_{i}\right. \text { at most once). }
\end{aligned}
$$

The proof of this proposition is found in section 7 .

Coarse Graining Estimate. The next step is to further bound the right-side in Proposition 4.3 through a detailed decomposition of visit times and locations in terms of an $\epsilon_{1}, \epsilon_{2}$-perturbed rate $\mathbb{J}_{\mathcal{U}_{0}, \epsilon_{1}, \epsilon_{2}}$.

Observe for $1 \leq i \leq N+M$ that the submatrix $\left(\bar{P}_{n, \epsilon_{1}, \epsilon_{2}}\right)_{C_{i}}=P\left(i, \epsilon_{1}, \epsilon_{2}\right)$ is independent of $n$ and

$$
P\left(i, \epsilon_{1}, \epsilon_{2}\right)=\left\{\begin{aligned}
\left(\epsilon_{1}\right) & \text { for } i \in \mathcal{D} \\
P\left(i, \epsilon_{2}\right) & \text { for } i \in \mathcal{G}
\end{aligned}\right.
$$

Denote the extended rate function $\mathbb{I}_{i, \epsilon_{1}, \epsilon_{2}}=\mathbb{I}_{C_{i}, f, P\left(i, \epsilon_{1}, \epsilon_{2}\right)}$ and associated domain of finiteness $Q_{i, \epsilon_{1}, \epsilon_{2}}=Q_{C_{i}, f, P\left(i, \epsilon_{1}, \epsilon_{2}\right)}$. In fact, explicitly when $i \in \mathcal{D}$

$$
\mathbb{I}_{i, \epsilon_{1}, \epsilon_{2}}(x)= \begin{cases}-\log \left(\epsilon_{1}\right) & \text { for } x=f\left(m_{i}\right)\left(\text { where } C_{i}=\left\{m_{i}\right\}\right) \\ \infty & \text { otherwise }\end{cases}
$$

and $\mathbb{I}_{i, \epsilon_{1}, \epsilon_{2}}(x)=\mathbb{I}_{i, \epsilon_{2}}(x)=\mathbb{I}_{C_{i}, f, P\left(i, \epsilon_{2}\right)}$ when $i \in \mathcal{G}$.

Recall now the object $C_{\vec{v}, U}$ near (2.6), and define for $\vec{v} \in \Omega_{N+M}, \vec{x} \in\left(\mathbb{R}^{d}\right)^{N+M}$, $\sigma \in \mathbb{S}_{N+M}$, and matrix $U=\{u(i, j): 1 \leq i, j \leq N+M\}$, the function

$$
C_{\vec{v}, U, \epsilon_{1}, \epsilon_{2}}(\sigma, \vec{x})=-\sum_{i=1}^{N+M-1}\left(\sum_{j=1}^{i} v_{j}\right) u(\sigma(i), \sigma(i+1))+\sum_{i=1}^{N+M} v_{i} \mathbb{I}_{\sigma(i), \epsilon_{1}, \epsilon_{2}}\left(x_{i}\right)
$$


when $N+M \geq 2$, and $C_{\vec{v}, U, \epsilon_{1}, \epsilon_{2}}(\sigma, \vec{x})=\mathbb{I}_{1, \epsilon_{1}, \epsilon_{2}}\left(x_{1}\right)$ when $N+M=1$. Define also, for $z \in \mathbb{R}^{d}$,

$$
\mathbb{J}_{U, \epsilon_{1}, \epsilon_{2}}(z)=\inf _{\vec{v} \in \Omega_{N+M}} \inf _{\vec{x} \in D(N+M, \vec{v}, z)} \min _{\sigma \in S_{N+M}} C_{\vec{v}, U, \epsilon_{1}, \epsilon_{2}}(\sigma, \vec{x}) .
$$

We comment, when $N=0$ and all $P(i)>0$ for $i \in \mathcal{G}$, that $\mathbb{J}_{U, \epsilon_{1}, \epsilon_{2}}=\mathbb{J}_{U}$ for all $\epsilon_{1}, \epsilon_{2}$ small, and so the following result would already give the desired upper bound.

Proposition 4.4 For $\epsilon_{1}, \epsilon_{2}>0$, we have

$$
\varlimsup_{n \rightarrow \infty} \frac{1}{n} \log \bar{\mu}_{\pi, \epsilon_{1}, \epsilon_{2}}\left(Z_{n} \in \bar{\Gamma}, \vec{X}_{n} \text { enters each } C_{i} \text { at most once }\right) \leq-\mathbb{J}_{\mathcal{U}_{0}, \epsilon_{1}, \epsilon_{2}}(\bar{\Gamma} \cap \mathbb{K}) .
$$

The proof of the proposition is in section 8 .

Limit Estimate on $\mathbb{J}_{\mathcal{U}_{0}, \epsilon_{1}, \epsilon_{2}}$. The last step is to analyze $\mathbb{J}_{\mathcal{U}_{0}, \epsilon_{1}, \epsilon_{2}}$ as $\epsilon_{1}, \epsilon_{2} \downarrow 0$ in the following proposition proved in section 10.

Proposition 4.5 We have

$$
\varlimsup_{\epsilon_{2} \downarrow 0} \varlimsup_{\epsilon_{1} \downarrow 0}-\mathbb{J}_{\mathcal{U}_{0}, \epsilon_{1}, \epsilon_{2}}(\bar{\Gamma} \cap \mathbb{K}) \leq-\mathbb{J}_{\mathcal{U}_{0}}(\bar{\Gamma}) .
$$

Now, putting together these results above gives Theorem 3.1.

\subsection{Lower Bounds: Proof of Theorem 3.2}

The argument is similar in structure to the upper bound. To prove part (i), a reduction is first made with respect to "initial ergodicity" which can be skipped if one is willing to assume $\mathbb{P}_{\pi}$ satisfies the stronger condition (SIE)-1 rather than just (SIE). Then, a "surgery of paths" estimate, a "homogeneous rest cost" comparison, and finally a "coarse graining cost" estimate are given. Last, having proved part (i), the second lower bound part (ii) is argued.

Let $\Gamma \subset \mathbb{R}^{d}$ be a Borel set. If $\Gamma^{o}=\emptyset$, the bound is trivial. Otherwise, let $x_{0} \in \Gamma^{o}$ and $\Gamma_{1}=B\left(x_{0}, a\right) \subset \Gamma^{o}$ be an open ball of radius $a>0$.

SIE Estimate. The following estimate shows that under condition (SIE), the first few transition kernels do not contribute effectively to lower bounds, and in particular, condition (SIE) may be replaced with (SIE-1). When $\mathbb{P}_{\pi}$ satisfies (SIE), let $P_{n}^{\prime}=P_{n+n_{1}}$ for $n \geq 1$, and let $\eta(l)=\mathbb{P}_{\pi}\left(X_{n_{1}}=l\right)$ for $l \in \Sigma$. Let also $\mathbb{P}_{\eta}^{\prime}$ be constructed with respect to $\left\{P_{n}^{\prime}\right\}$ and distribution $\eta$. Clearly, we have $n_{0}\left(\left\{P_{n}^{\prime}\right\}\right)=1$ and $\mathbb{P}_{\eta}^{\prime}$ satisfies (SIE- 1$)$.

Proposition 4.6 Let $\Gamma_{2}=B\left(x_{0}, a / 2\right)$ and suppose $\mathbb{P}_{\pi}$ satisfies (SIE). Then, we have

$$
\underline{\lim } \frac{1}{n} \log \mathbb{P}_{\pi}\left(Z_{n} \in \Gamma_{1}\right) \geq \underline{\lim } \frac{1}{n} \log \mathbb{P}_{\eta}^{\prime}\left(Z_{n} \in \Gamma_{2}\right) .
$$


Proof. Note that

$$
\left\{Z_{n} \in B\left(x_{0}, a\right)\right\} \supset\left\{\frac{n-n_{1}}{n} Z_{n_{1}+1}^{n} \in B\left(x_{0}, a-c_{1} / n\right)\right\} .
$$

where $c_{1}=n_{1}\|f\|$. Then,

$$
\begin{aligned}
\mathbb{P}_{\pi}\left(Z_{n} \in \Gamma_{1}\right) & \geq \mathbb{P}_{\pi}\left(\left(\left(n-n_{1}\right) / n\right) Z_{n_{1}+1}^{n} \in B\left(x_{0}, a-c_{1} / n\right)\right) \\
& =\sum_{l \in \Sigma} \eta(l) \mathbb{P}_{\left(n_{1}, l\right)}\left(\left(\left(n-n_{1}\right) / n\right) Z_{n_{1}+1}^{n} \in B\left(x_{0}, a-c_{1} / n\right)\right) \\
& =\mathbb{P}_{\eta}^{\prime}\left(Z_{n-n_{1}} \in\left(n /\left(n-n_{1}\right)\right) B\left(x_{0}, a-c_{1} / n\right)\right) .
\end{aligned}
$$

The proposition now follows by simple calculations.

In view of the last proposition, with regard to the standard lower bound methods, we may just as well assume that $\mathbb{P}_{\pi}$ satisfies (SIE-1) if (SIE) already holds.

Surgery of Paths Estimate. We underestimate $\mathbb{P}_{\pi}$ by another measure $\check{\mu}_{\pi, \epsilon_{1}, \epsilon_{2}}$ whose connection transitions correspond to $\mathcal{T}_{1}$. Slightly different from the surgery for the upper bound, the paths focused upon here are those which make at most one "long" visit to sets $\left\{C_{i}: i \in \mathcal{G}\right\}$, but travel between them in "short" trips through all $\left\{C_{i}: 1 \leq i \leq N+M\right\}$.

Let $E(N, M)=(M-1) E_{0}(N, M)$, and recall the connecting weight $\underline{\gamma}^{1}(n,(i, j))$ for distinct $i, j \in \mathcal{G}$ (cf. (2.10)). Define

$$
\check{\gamma}^{0}(n,(i, j))=\min _{0 \leq k \leq E(N, M)} \underline{\gamma}^{1}(n+k,(i, j))
$$

which picks the smallest weight in a traveling frame.

Define also $\check{\mathcal{P}}_{n}=\left\{\check{p}_{n}(s, t)\right\}$ for $n \geq 1$ by

$\check{p}_{n}(s, t)=\left\{\begin{aligned} \check{\gamma}^{0}(n,(i, j)) & \text { for all } s \in C_{i}, t \in C_{j} \text {, and distinct } i, j \in \mathcal{G} \\ p_{n}(s, t) & \text { for } s, t \in C_{i} \text { and } i \in \mathcal{G}, \text { or } s \in C_{i}, t \in C_{j} \text { when } i \text { or } j \in \mathcal{D} .\end{aligned}\right.$

Let $\check{\mu}_{\pi}$ be made through Construction $(\mathrm{CON})$ with $\left\{\check{\mathcal{P}}_{n}\right\}$ and $\pi$.

In addition, for convenience, let

$$
G_{n}=\left\{\vec{X}_{n} \text { enters only }\left\{C_{i}: i \in \mathcal{G}\right\} \text { with at most one visit to each set }\right\} .
$$

Proposition 4.7 Let $\Gamma_{3}=B\left(x_{0}, a / 4\right)$ and suppose $\mathbb{P}_{\pi}$ satisfies (SIE)-1. Then,

$$
\underline{\lim } \frac{1}{n} \log \mathbb{P}_{\pi}\left(Z_{n}(f) \in \Gamma_{2}\right) \geq \underline{\lim } \frac{1}{n} \log \check{\mu}_{\pi}\left(Z_{n}(f) \in \Gamma_{3}, G_{n}\right) .
$$

The proof is given in section 6 .

Homogeneous Rest Cost Comparision. As before, we compare $\check{\mu}_{\pi}$ with a measure $\underline{\mu}_{\pi}$ which replaces "nonhomogeneous" transitions within sets $C_{i}$ by "limiting homogeneous" transition weights. 
Define $\underline{\mathcal{P}}_{n}=\left\{\underline{p}_{n}(s, t)\right\}$ for $n \geq 1$ by

$$
\underline{p}_{n}(s, t)=\left\{\begin{aligned}
\check{\gamma}^{0}(n,(i, j)) & \text { for all } s \in C_{i}, t \in C_{j}, \text { and distinct } i, j \in \mathcal{G} \\
p(s, t) & \text { for } s, t \in C_{i} \text { and } i \in \mathcal{G} \\
0 & \text { otherwise. }
\end{aligned}\right.
$$

Correspondingly, define $\underline{\mu}_{\pi}$ through Construction (CON) with $\left\{\underline{\mathcal{P}}_{n}\right\}$ and initial distribution $\pi$.

Proposition 4.8 Suppose $n_{0}\left(\left\{P_{n}\right\}\right)=1$. Then, we have

$$
\underline{\lim } \frac{1}{n} \log \check{\mu}_{\pi}\left(Z_{n} \in \Gamma_{3}, G_{n}\right) \geq \underline{\lim } \frac{1}{n} \log \underline{\mu}_{\pi}\left(Z_{n} \in \Gamma_{3}, G_{n}\right) .
$$

The proof is in section 7 .

Coarse Graining Estimate. Again, we bound the right-side above through a decomposition of visit times and locations.

Proposition 4.9 Let $\pi$ be (SIE-1)-positive. Then,

$$
\underline{\lim } \frac{1}{n} \log \underline{\mu}_{\pi}\left(Z_{n} \in \Gamma_{3}, G_{n}\right) \geq-\mathbb{J}_{\mathcal{T}_{1}}\left(\Gamma_{3}\right) .
$$

The proof is in section 9 .

Finally, as $x_{0} \in \Gamma^{o}$ is arbitrary, we have that

$$
\varliminf_{n \rightarrow \infty} \frac{1}{n} \log \mathbb{P}_{\pi}\left(Z_{n} \in \Gamma^{o}\right) \geq-\inf _{z \in \Gamma^{o}} \mathbb{J}_{\mathcal{T}_{1}}(z)
$$

and so part (i) is proved.

Proof of Theorem 3.2 Part (ii). The following cost bound, proved in section 11 , is the key step.

Proposition 4.10 We have under Assumptions $B$ or $C$ that $\mathcal{T}_{1} \geq \mathcal{T}_{0}$ and so $\mathbb{J}_{\mathcal{T}_{1}} \leq \mathbb{J}_{\mathcal{T}_{0}}$.

Therefore, given the lower bound in part (i), the second part follows directly.

\section{$5 \quad$ Path Surgery Upper Bound}

The strategy of Proposition 4.2 is to compare the probability of a path which moves many times between sets with that of a respective "rearranged" path with less sojourns. To make estimates we will need a few more definitions.

Let $\underline{t}(n)$ be the largest entry which connects "upwards" with respect to the ordering of the sets $\left\{C_{i}\right\}$ in the canonical decomposition of $P$,

$$
\underline{t}(n)=\max _{1 \leq j<i \leq N+M} \hat{t}(n,(i, j)) .
$$


Observe, as "movement up the tree is impossible in the limit," or more precisely as $T_{n}(i, j)$ vanishes for $1 \leq j<i \leq N+M$, we have $\underline{t}(n) \rightarrow 0$ as $n \rightarrow \infty$.

Define also for $\epsilon_{1}, \epsilon_{2} \geq 0$, the matrix $\tilde{P}_{n, \epsilon_{1}, \epsilon_{2}}=\left\{\tilde{p}_{n, \epsilon_{1}, \epsilon_{2}}(s, t)\right\}$ by

$$
\tilde{p}_{n, \epsilon_{1}, \epsilon_{2}}(s, t)= \begin{cases}\hat{t}(n,(i, j)) & \text { for } s \in C_{i}, t \in C_{j} \text { and distinct } 1 \leq i, j \leq N+M \\ p_{n}\left(s, t ; \epsilon_{2}\right) & \text { for } s, t \in C_{i} \text { and } i \in \mathcal{G} \\ p_{n}\left(s, t ; \epsilon_{1}\right) & \text { for } s, t \in C_{i} \text { and } i \in \mathcal{D}\end{cases}
$$

for $n \geq 1$. Form now through Construction $(\mathrm{CON})$ the measure $\nu_{\pi, \epsilon_{1}, \epsilon_{2}}$ with respect to initial distribution $\pi$ and transition matrices $\left\{\tilde{P}_{n, \epsilon_{1}, \epsilon_{2}}\right\}$.

Let also $\tilde{p}=\min \left\{\epsilon_{1}, \epsilon_{2}\right\}$ and observe that $\tilde{p}$ is less than the minimum transition probability within subblocks,

$$
\tilde{p} \leq \min _{1 \leq l \leq N+M} \min _{s, t \in C_{l}} \tilde{p}_{n, \epsilon_{1}, \epsilon_{2}}(s, t) .
$$

We now describe a procedure to cut paths into "resting" and "traveling" parts which will then be rearranged through a "rearrangement map." Let $\vec{x}_{n}=\left\langle x_{1}, \ldots, x_{n}\right\rangle \in \Sigma^{n}$ be a path of length $n \geq 2$. We will say that $\vec{x}_{n}$ possesses a "switch" at time $1 \leq i \leq n-1$ if $x_{i} \in C_{j}$ and $x_{i+1} \in C_{k}$ for $j \neq k$. For a path $\vec{x}_{n}$ which switches $l \geq 1$ times, let $g_{k}\left(\vec{x}_{n}\right)$ be the time of the $k$ th switch where $1 \leq k \leq l$. Set also $g_{0}\left(\vec{x}_{n}\right)=0$ and $g_{l+1}\left(\vec{x}_{n}\right)=n$.

Define now, for $1 \leq k \leq l$, the "path segments" between switch times,

$J_{k}\left(\vec{x}_{n}\right)=\left\langle x_{g_{k-1}\left(\vec{x}_{n}\right)+1}, \ldots, x_{g_{k}\left(\vec{x}_{n}\right)}\right\rangle$, and the remainder $J_{l+1}\left(\vec{x}_{n}\right)=\left\langle x_{g_{l}\left(\vec{x}_{n}\right)+1}, \ldots, x_{n}\right\rangle$.

Define also that $J_{k, 2}\left(\vec{x}_{n}\right)=\left\langle x_{g_{k-1}\left(\vec{x}_{n}\right)+2}, \ldots, x_{g_{k}\left(\vec{x}_{n}\right)}\right\rangle$ when $g_{k}\left(\vec{x}_{n}\right) \geq g_{k-1}\left(\vec{x}_{n}\right)+2$.

In addition, let $C_{i_{k}}$ be the subset in which path $J_{k}$ lies for $1 \leq k \leq l+1$, and let $\mathcal{C}_{l}=\mathcal{C}_{l}\left(\vec{x}_{n}\right)=\left\langle C_{i_{1}}, \ldots, C_{i_{l+1}}\right\rangle$ be the sequence of subsets visited, in the order of visitation. Also, let $\left\|\mathcal{C}_{l}\right\|$ be the number of distinct elements in $\mathcal{C}_{l}$. We will say $\vec{x}_{n}$ has no "repeat visits" if the sequence $\mathcal{C}_{l}$ contains no repetitions.

For $0 \leq k \leq n-1$ and $1 \leq j \leq N+M$, define the sets,

$$
\begin{aligned}
& A_{n}(k)=\left\{\vec{x}_{n}: \vec{x}_{n} \text { switches } k \text { times }\right\} \text { and } \\
& A_{n}^{\prime}(j)=\left\{\vec{x}_{n}: \vec{x}_{n} \text { switches } j \text { times, with no repeat visits }\right\} .
\end{aligned}
$$

When there are at least two sets, $N+M \geq 2$, we define the map

$$
\sigma_{l}: A_{n}(l) \rightarrow \cup_{j=1}^{\min \{N+M-1, l\}} A_{n}^{\prime}(j),
$$

for $l \geq 1$, in the following steps.

1. Let $\vec{x}_{n} \in A_{n}(l)$. Let $s_{\left\|\mathcal{C}_{l}\right\|}=l+1$ and $s_{\left\|\mathcal{C}_{l}\right\|-1}=l$. Inductively define, for $k<\left\|\mathcal{C}_{l}\right\|$,

$$
s_{k}=\max \left\{j: C_{i_{j}} \notin\left\{C_{i_{s_{k+1}}}, C_{i_{s_{k+2}}}, \ldots, C_{i_{s_{\| \mathcal{C}} \|}}\right\}\right\} .
$$


In words, $C_{i_{s_{\| \mathcal{C}} \|}}, \ldots, C_{i_{s_{1}}}$ are the $\left\|\mathcal{C}_{l}\right\|$ distinct subsets visited in reverse order starting from the last state of $\vec{x}_{n}$.

2. For $1 \leq k \leq\left\|\mathcal{C}_{l}\right\|$, let $J_{\alpha_{1}^{k}}, \ldots, J_{\alpha_{d_{k}}^{k}}$, where $\alpha_{1}^{k}<\cdots<\alpha_{d_{k}}^{k}=s_{k}$, be the $d_{k} \geq 1$ paths which lie in $C_{i_{s_{k}}}$.

3. Define

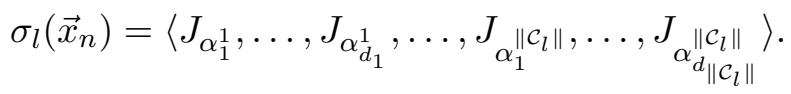

In words, $\sigma_{l}$ rearranges the paths corresponding to distinct subsets so that the reverse visiting order is preserved. We comment that the last path $J_{\alpha_{d_{l+1}}^{l+1}}$ is preserved under $\sigma_{l}$, and that $\sigma_{1}$ is the identity map.

Example 1. Suppose $N+M=8$, and $\vec{x}_{n} \in A_{n}(25)$ where

$$
\mathcal{C}_{25}=\left\langle C_{8}, C_{6}, C_{8}, C_{7}, C_{5}, C_{7}, C_{6}, C_{5}, C_{6}, C_{4}, C_{2}, C_{4}, C_{3}, C_{1}, C_{3}, C_{1}, C_{2}, C_{1}, C_{6}, C_{7}, C_{5}, C_{4}, C_{2}, C_{5}, C_{2}, C_{4}\right\rangle .
$$

Here, $\|\mathcal{C}\|=8$, and $s_{1}=3, s_{2}=15, s_{3}=18, s_{4}=19, s_{5}=20, s_{6}=24, s_{7}=25, s_{8}=26$. Then,

$$
\begin{gathered}
\left\langle C_{i_{s_{1}}}, C_{i_{s_{2}}}, C_{i_{s_{3}}}, C_{i_{s_{4}}}, C_{i_{s_{5}}}, C_{i_{s_{6}}}, C_{i_{s_{7}}}, C_{i_{s_{8}}}\right\rangle=\left\langle C_{8}, C_{3}, C_{1}, C_{6}, C_{7}, C_{5}, C_{2}, C_{4}\right\rangle \quad \text { and } \\
\sigma_{25}\left(\vec{x}_{n}\right)=\left\langle J_{1}, J_{3}, J_{13}, J_{15}, J_{14}, J_{16}, J_{18}, J_{2}, J_{7}, J_{9},\right. \\
\left.J_{19}, J_{4}, J_{6}, J_{20}, J_{5}, J_{8}, J_{21}, J_{24} J_{11}, J_{17} J_{23}, J_{25}, J_{10}, J_{12}, J_{22}, J_{26}\right\rangle .
\end{gathered}
$$

Finally, we recall at this point useful versions of the "union of events" bound.

Lemma 5.1 Let $N \geq 1$ and let $\left\{a_{n}^{i}: i, n \geq 1\right\}$ be an array of non-negative numbers. We have then

$$
\begin{aligned}
& \varlimsup_{n \rightarrow \infty} \frac{1}{n} \log \sum_{i=1}^{N} a_{n}^{i}=\max _{1 \leq i \leq N} \varlimsup_{n \rightarrow \infty} \frac{1}{n} \log a_{n}^{i} \text { and } \\
& \varliminf_{n \rightarrow \infty} \frac{1}{n} \log \sum_{i=1}^{N} a_{n}^{i}=\underline{\lim _{1 \leq i \leq N}} \max _{1 \leq} \frac{1}{n} \log a_{n}^{i} \geq \max _{1 \leq i \leq N} \underline{\lim } \frac{1}{n \rightarrow \infty} \log a_{n}^{i} .
\end{aligned}
$$

In addition, let $\alpha \geq 1$ be an integer, and let $\{\beta(n)\}$ be a sequence where $\beta(n) \leq n^{\alpha}$ for $n \geq 1$. Then,

$$
\varlimsup_{n \rightarrow \infty} \frac{1}{n} \log \sum_{i=1}^{\beta(n)} a_{n}^{i}=\varlimsup_{n \rightarrow \infty} \max _{1 \leq i \leq \beta(n)} \frac{1}{n} \log a_{n}^{i}
$$

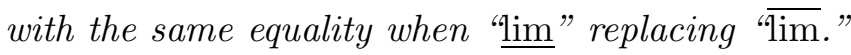

See [10] Lemma 1.2.15, for the "im" proof. The other statements follow similarly.

Proof of Proposition 4.2. As $P_{n} \leq \tilde{P}_{n, \epsilon_{1}, \epsilon_{2}}$ elementwise, we have

$$
\mathbb{P}_{\pi}\left(Z_{n} \in \bar{\Gamma}\right) \leq \nu_{\pi, \epsilon_{1}, \epsilon_{2}}\left(Z_{n} \in \bar{\Gamma}\right) .
$$


Now consider the case $N+M=1$ when $P$ corresponds to one irreducible set $C_{1}=\Sigma$. Trivially in this case $\vec{X}_{n}$ does not leave $C_{1}$ and so more than one switch is impossible. Therefore, the upper bound statement holds immediately.

We will now assume that $N+M \geq 2$. By Lemma 5.1,

$$
\varlimsup \frac{1}{n} \log \mathbb{P}_{\pi}\left(Z_{n} \in \Gamma\right) \leq \max _{\substack{x_{0} \in \Sigma \\ \pi\left(x_{0}\right)>0}} \varlimsup \frac{1}{n} \log \nu_{x_{0}, \epsilon_{1}, \epsilon_{2}}\left(Z_{n} \in \bar{\Gamma}\right) .
$$

Hence, it suffices to focus on $\nu_{x_{0}, \epsilon_{1}, \epsilon_{2}}$ for a given $x_{0} \in \Sigma$ such that $\pi\left(x_{0}\right)>0$.

The main idea exploited now is that for a realization $\vec{X}_{n}$ which switches between sets $\left\{C_{i}\right\}$ many times there will be guaranteed a large number of these switches "up the tree" between sets $C_{i}$ and $C_{j}$ for $i>j$ whose chance is small, and so such paths are unlikely. For notational simplicity, we now suppress $\epsilon_{1}, \epsilon_{2}$ subscripts.

Step 1. Decompose according to the number of switches,

$$
\nu_{x_{0}}\left(Z_{n} \in \Gamma\right)=\sum_{i=0}^{n-1} \nu_{x_{0}}\left(Z_{n} \in \Gamma, A_{n}(i)\right) .
$$

Step 2. Let $l \geq 1$ and let $\vec{x}_{n} \in\left\{Z_{n} \in \Gamma\right\} \cap A_{n}(l)$. Let also $\vec{y}_{n} \in \sigma_{l}^{-1}\left(\sigma_{l}\left(\vec{x}_{n}\right)\right)$, that is $\vec{y}_{n}$ is a path with $l$ switches which rearranges to $\sigma_{l}\left(\vec{x}_{n}\right)$. As $\vec{y}_{n}=\left\langle J_{1}\left(\vec{y}_{n}\right), \ldots, J_{l+1}\left(\vec{y}_{n}\right)\right\rangle$ where $J_{k}\left(\vec{y}_{n}\right)$ is a path in $C_{i_{k}}$ for $1 \leq k \leq l+1$, we have

$$
\begin{aligned}
& \nu_{x_{0}}\left(\vec{X}_{n}=\vec{y}_{n}\right) \\
& =\nu_{x_{0}}\left(\vec{X}_{1}^{g_{1}}=J_{1}\right) \prod_{\substack{1 \leq k \leq l \\
g_{k} \geq g_{k-1}+2}} \nu_{\left(g_{k}+1, y_{g_{k}+1}\right)}\left(\vec{X}_{g_{k}+2}^{g_{k+1}}=J_{k+1,2}\right) \prod_{k=1}^{l} \hat{t}\left(g_{k}+1,\left(i_{k}, i_{k+1}\right)\right)
\end{aligned}
$$

where $g_{k}=g_{k}\left(\vec{y}_{n}\right)$ and $J_{k+1,2}=J_{k+1,2}\left(\vec{y}_{n}\right)$, defined above, are shortened for clarity.

We now bound the right-side of (5.3) by

$$
\begin{aligned}
& \left(\tilde{p}_{1}\left(x_{0}, y_{1}\right) / 1\right) \hat{\mu}_{x_{0}}\left(\vec{X}_{n}=\sigma_{l}\left(\vec{x}_{n}\right)\right) \\
& \cdot \prod_{k=1}^{l} \hat{t}\left(g_{k}\left(\vec{y}_{n}\right)+1,\left(i_{k}, i_{k+1}\right)\right) \prod_{k=1}^{\left\|\mathcal{C}_{l}\right\|-1} \gamma^{-1}\left(g_{k}\left(\sigma_{l}\left(\vec{x}_{n}\right)\right)+1,\left(i_{s_{k}}, i_{s_{k+1}}\right)\right) \cdot(1 / \tilde{p})^{l-\left(\left\|\mathcal{C}_{l}\right\|-1\right)} .
\end{aligned}
$$

This bound (5.4) is explained by first recalling that in $\sigma_{l}\left(\vec{x}_{n}\right)$ there are $\left\|\mathcal{C}_{l}\right\|-1$ connections between different sets $\left\{C_{i}\right\}$. The expression (5.3) is then multiplied and divided by corresponding connection probabilities with respect to $\hat{\mu}_{x_{0}}$ giving the " $\prod \gamma^{-1}(\cdots)$ " term. Second, the prefactor, $\left(\tilde{p}_{1}\left(x_{0}, y_{1}\right) / 1\right) \leq 1$ arises in connecting $x_{0}$ to the first state of $\sigma_{l}\left(\vec{x}_{n}\right)$ with respect to $\hat{\mu}_{x_{0}}$ and noting the constant form of $\hat{\mathcal{P}}_{1}$. Third, in forming $\sigma_{l}\left(\vec{x}_{n}\right)$ from $\vec{y}_{n}$, with respect to $\nu_{x_{0}}, l-\left\|\mathcal{C}_{l}\right\|+1$ connections between different sets are replaced by corresponding internal transition probabilities and divided by them. These $l-\left\|\mathcal{C}_{l}\right\|+1$ divisors are then underestimated by the product of $\tilde{p}$ 's. 
Step 3. We now bound further the product terms in (5.4). Consider the subproduct

$$
\prod_{k=s_{r}}^{s_{r+1}-1} \hat{t}\left(g_{k}\left(\vec{y}_{n}\right)+1,\left(i_{k}, i_{k+1}\right)\right)
$$

whose factors correspond to transitions between sets in subsequence $\left\langle C_{i_{s_{r}}}, \ldots, C_{i_{s_{r+1}}}\right\rangle$ for $1 \leq r \leq\left\|\mathcal{C}_{l}\right\|-1$. From this subsequence, we derive a smaller subsequence in the following algorithm.

1. Let $\beta_{1}^{r}$ be the smallest index $s_{r}+1 \leq q \leq s_{r+1}$ such that $C_{i_{q}}=C_{i_{s_{r+1}}}$.

2. If $\beta_{1}^{r}>s_{r}+1$, let $\beta_{2}^{r}$ be the smallest index $s_{r}+1 \leq q \leq \beta_{1}^{r}-1$ such that $C_{i_{q}}=C_{i_{\beta_{1}^{r}-1}}$. Otherwise, stop.

3. Continue iteratively: If $\beta_{m}^{r}>s_{r}+1$, let $\beta_{m+1}^{r}$ be the smallest index $s_{r}+1 \leq$ $q \leq \beta_{m}^{r}-1$ such that $C_{i_{q}}=C_{i_{\beta_{m}^{r}-1}}$. Otherwise, stop. Recalling the definition of $s_{r}$, there are at most $\left\|\mathcal{C}_{l}\right\|-r$ distinct sets in the sequence $\left\langle C_{i_{s_{r}}}, \ldots, C_{i_{s_{r+1}}}\right\rangle$. Therefore, the above process finishes in $n(r) \leq\left\|\mathcal{C}_{l}\right\|-r$ steps to find $\beta_{n(r)}^{r}=s_{r}+1$.

Example 2. With respect to the path $\vec{x}_{n}$ in Example 1, we consider the algorithm for $r=1$. We saw that $s_{1}=3$ and $s_{2}=15$, and

$$
\left\langle C_{i_{s_{1}}}, C_{i_{s_{1}+1}} \ldots, C_{i_{s_{2}}}\right\rangle=\left\langle C_{8}, C_{7}, C_{5}, C_{7}, C_{6}, C_{5}, C_{6}, C_{4}, C_{2}, C_{4}, C_{3}, C_{1}, C_{3}\right\rangle
$$

Here, there are $n(1)=4$ distinct sets, and $\beta_{1}^{1}=s_{1}+10$ is the smallest index so that $C_{i_{q}}=C_{3}$. Similarly, $\beta_{2}^{1}=s_{1}+7$ is smallest where $C_{i_{q}}=C_{i_{s_{1}}+9}=C_{4}$. Also, $\beta_{3}^{1}=s_{1}+4$ and $\beta_{4}^{1}=s_{1}+1$.

By construction, the terms

$\hat{t}\left(g_{s_{r}}\left(\vec{y}_{n}\right)+1,\left(i_{s_{r}}, i_{\beta_{n(r)}^{r}}\right)\right), \hat{t}\left(g_{\beta_{n(r)}^{r}}\left(\overrightarrow{y_{n}}\right)+1,\left(i_{\beta_{n(r)}^{r}}, i_{\beta_{n(r)-1}^{r}}\right)\right), \ldots, \hat{t}\left(g_{\beta_{2}^{r}}\left(\overrightarrow{y_{n}}\right)+1,\left(i_{\beta_{2}^{r}}, i_{\beta_{1}^{r}}\right)\right)$

all appear as factors in (5.5). Also, by monotonicity of $\hat{t}(n,(i, j))$,

$$
\begin{aligned}
& \hat{t}\left(g_{s_{r}}\left(\vec{y}_{n}\right)+1,\left(i_{s_{r}}, i_{\beta_{n(r)}^{r}}\right)\right) \prod_{k=1}^{n(r)-1} \hat{t}\left(g_{\beta_{k+1}^{r}}\left(\overrightarrow{y_{n}}\right)+1,\left(i_{\beta_{k+1}^{r}}, i_{\beta_{k}^{r}}\right)\right) \\
& \quad \leq \hat{t}\left(g_{s_{r}}\left(\vec{y}_{n}\right)+1,\left(i_{s_{r}}, i_{\beta_{n(r)}^{r}}\right)\right) \prod_{k=1}^{n(r)-1} \hat{t}\left(g_{s_{r}}\left(\overrightarrow{y_{n}}\right)+n(r)-k+1,\left(i_{\beta_{k+1}^{r}}, i_{\beta_{k}^{r}}\right)\right) .
\end{aligned}
$$

Also, by construction, the $r$ th switch time between sets $C_{i_{s_{r}}}$ and $C_{i_{s_{r+1}}}$ in the rearranged path $\sigma_{l}\left(\vec{x}_{n}\right)$ is less than the last time to switch to $C_{i_{s_{r+1}}}$ in path $\vec{y}_{n}$,

$$
g_{r}\left(\sigma_{l}\left(\vec{x}_{n}\right)\right) \leq g_{s_{r}}\left(\vec{y}_{n}\right)
$$

So, by monotonicity again,

the right-side of $(5.6)$ is bounded above by $\gamma\left(g_{r}\left(\sigma_{l}\left(\vec{x}_{n}\right)\right)+1,\left(i_{s_{r}}, i_{s_{r+1}}\right)\right)$. 
Also, in particular, it will be convenient to note the gross bound, as $\hat{t}(n,(i, j)) \leq 1$ applies to those terms in (5.5) not covered by (5.6), that $\prod_{k=s_{r}}^{s_{r+1}-1} \hat{t}\left(g_{k}\left(\vec{y}_{n}\right)+1,\left(i_{k}, i_{k+1}\right)\right) \leq$ $\gamma\left(g_{r}\left(\sigma_{l}\left(\vec{x}_{n}\right)\right)+1,\left(i_{s_{r}}, i_{s_{r+1}}\right)\right)$ and so,

$$
\prod_{k=1}^{l} \hat{t}\left(g_{k}\left(\vec{y}_{n}\right)+1,\left(i_{k}, i_{k+1}\right)\right) \leq \prod_{k=1}^{\left\|\mathcal{C}_{l}\right\|-1} \gamma\left(g_{k}\left(\sigma_{l}\left(\vec{x}_{n}\right)\right)+1,\left(i_{s_{k}}, i_{s_{k+1}}\right)\right) .
$$

Step 4. We now consider cases when $l$ is "large" and "small." Suppose first that $l$ is small, namely $\left.l \leq\left\|\mathcal{C}_{l}\right\|\left(\left\|\mathcal{C}_{l}\right\|-1\right) / 2\right)+N+M-1$. Then, we have the bound, noting (5.3), (5.4) and (5.7), that

$$
\nu_{x_{0}}\left(\vec{X}_{n}=\vec{y}_{n}\right) \leq(1 / \tilde{p})^{l} \hat{\mu}_{x_{0}}\left(\vec{X}_{n}=\sigma_{l}\left(\vec{x}_{n}\right)\right) .
$$

Suppose now that $l$ is large, that is $\left.l>\left\|\mathcal{C}_{l}\right\|\left(\left\|\mathcal{C}_{l}\right\|-1\right) / 2\right)+N+M-1$. As the chain can only make at most $N+M-1$ consecutive "downward" switches, that is from sets $C_{i}$ to $C_{j}$ for $i<j$, in $q>N+M-1$ switches there will be at least $[q /(N+M-1)]$ "upward" switches from sets $C_{i}$ to $C_{j}$ for $i>j$.

As $n(k) \leq\left\|\mathcal{C}_{l}\right\|-k$, and so $\left.\sum_{k=1}^{\left\|\mathcal{C}_{l}\right\|-1} n(k) \leq\left\|\mathcal{C}_{l}\right\|\left(\left\|\mathcal{C}_{l}\right\|-1\right) / 2\right)$, we see carefully in Step 3 that one takes at most $\left\|\mathcal{C}_{l}\right\|\left(\left\|\mathcal{C}_{l}\right\|-1\right) / 2$ factors from $\prod_{k=1}^{l} \hat{t}\left(g_{k}\left(\vec{y}_{n}\right)+1,\left(i_{k}, i_{k+1}\right)\right)$ whose product is then dominated by $\prod_{k=1}^{\left\|C_{l}\right\|-1} \gamma_{k}\left(\sigma_{l}\left(\vec{x}_{n}\right)+1,\left(i_{s_{k}}, i_{s_{k+1}}\right)\right)$. Hence, remaining in the original product are at least $l-\left\|\mathcal{C}_{l}\right\|\left(\left\|\mathcal{C}_{l}\right\|-1\right) / 2$ uncommitted factors of which at least

$$
\underline{l}=\left\lfloor\left(l-\left\|\mathcal{C}_{l}\right\|\left(\left\|\mathcal{C}_{l}\right\|-1\right) / 2\right) /(N+M-1)\right\rfloor
$$

correspond to upward transitions.

Then, using monotonicity of $\underline{t}(n)$, we have

$$
\prod_{k=1}^{l} \hat{t}\left(g_{k}\left(\vec{y}_{n}\right)+1,\left(i_{k}, i_{k+1}\right)\right) \leq \prod_{k=1}^{\left\|C_{l}\right\|-1} \gamma_{k}\left(\sigma_{l}\left(\vec{x}_{n}\right)+1,\left(i_{s_{k}}, i_{s_{k+1}}\right)\right) \prod_{j=1}^{\underline{l}} \underline{t}(j) .
$$

Further, noting (5.3) and (5.4), we have for $l$ "large,"

$$
\nu_{x_{0}}\left(\vec{X}_{n}=\vec{y}_{n}\right) \leq(1 / \tilde{p})^{l}\left[\prod_{j=1}^{\underline{l}} \underline{t}(j)\right] \hat{\mu}_{x_{0}}\left(\vec{X}_{n}=\sigma_{l}\left(\vec{x}_{n}\right)\right) .
$$

Step 5. We now estimate the size of the set $\sigma_{l}^{-1}\left(\sigma_{l}\left(\vec{x}_{n}\right)\right)$. Observe that the ordering of states within the $l+1$ subpaths in $\sigma_{l}\left(\vec{x}_{n}\right)$ is preserved among the paths $\sigma_{l}^{-1}\left(\sigma_{l}\left(\vec{x}_{n}\right)\right)$ with $l$ switches. Then, to overestimate $\left|\sigma_{l}^{-1}\left(\sigma_{l}\left(\vec{x}_{n}\right)\right)\right|$, we need only specify in what sequence the pairwise distinct sets, $C_{j_{1}} \neq C_{j_{2}} \neq \cdots \neq C_{j_{l+1}}$, are visited, and how long each visit takes since once the ordering of the sets and switch times are fixed, the arrangement within the $l+1$ subpaths is determined. 
A simple overcount of this procedure yields that

$$
\left|\sigma_{l}^{-1}\left(\sigma_{l}\left(\vec{x}_{n}\right)\right)\right| \leq\left(\begin{array}{c}
n \\
l
\end{array}\right) M^{l+1} .
$$

Therefore, from (5.8) and (5.9) we have that

$$
\nu_{x_{0}}\left(\vec{X}_{n} \in \sigma_{l}^{-1}\left(\sigma_{l}\left(\vec{x}_{n}\right)\right)\right) \leq\left\{\begin{aligned}
\left(\begin{array}{c}
n \\
l
\end{array}\right) M^{l+1} \tilde{p}^{-l} \hat{\mu}_{x_{0}}\left(\vec{X}_{n}=\sigma_{l}\left(\vec{x}_{n}\right)\right) & \text { for } l \text { "small" } \\
\left(\begin{array}{c}
n \\
l
\end{array}\right) M^{l+1} \tilde{p}^{-l}\left[\prod_{i=1}^{\underline{l}} \underline{t}(i)\right] \hat{\mu}_{x_{0}}\left(\vec{X}_{n}=\sigma_{l}\left(\vec{x}_{n}\right)\right) & \text { for } l \text { "large." }
\end{aligned}\right.
$$

Step 6. By Stirling's formula,

$$
\frac{1}{n} \log \left(\begin{array}{l}
n \\
l
\end{array}\right)=o(1)-\frac{l}{n} \log \left(\frac{l}{n}\right)-\frac{n-l}{n} \log \left(\frac{n-l}{n}\right) .
$$

With this estimate, we now analyze the factor, $\left(\begin{array}{c}n \\ l\end{array}\right) M^{l} \prod \frac{l}{1} \underline{t}(i)$, in (5.10). We consider cases when $l=o(n)$ and when $l \leq n$ is otherwise.

Case 1: $l=l_{n}=o(n)$. Then, $\log \left(\begin{array}{l}n \\ l_{n}\end{array}\right) / n \rightarrow 0$. Also, $M^{l_{n}}=e^{o(n)}, \tilde{p}^{-l_{n}}=e^{o(n)}$, and $\prod_{i=1}^{\underline{l}_{n}} \underline{t}(i)=e^{o(n)}$.

Case 2: $l=l_{n}$ satisfies $\varlimsup l_{n} / n \geq \epsilon$ for some $0<\epsilon \leq 1$. Let $n^{\prime}$ be a maximal subsequence. Then, $\left(\log \left(\begin{array}{c}n^{\prime} \\ l_{n^{\prime}}\end{array}\right) / n^{\prime}=O(1),\left(\log M^{l_{n^{\prime}}}\right) / n^{\prime} \leq 1+\log M,\left(\log \tilde{p}^{-l_{n^{\prime}}}\right) / n^{\prime} \leq 1+\right.$ $\log \tilde{p}^{-1}$. But, as $\underline{t}\left(n^{\prime}\right) \downarrow 0$, and $\varlimsup \underline{l}_{n}^{\prime} / n^{\prime} \geq \epsilon /(N+M-1)$, we have $\log \left[\prod_{i=1}^{\underline{l} \underline{n}^{\prime}} \underline{t}(i)\right] / n^{\prime} \rightarrow$ $-\infty$ as $n^{\prime} \rightarrow \infty$.

Therefore, with respect to a $C_{n}=e^{o(n)}$, independent of $l \geq 1$ and the path, we have from (5.10) that

$$
\nu_{x_{0}}\left(\vec{X}_{n} \in \sigma_{l}^{-1}\left(\sigma_{l}\left(\vec{x}_{n}\right)\right)\right) \leq C_{n} \hat{\mu}_{x_{0}}\left(\vec{X}_{n}=\sigma_{l}\left(\vec{x}_{n}\right)\right) .
$$

Step 7. Let $l \geq 1$, and let $\bar{A}_{n}(l)=\cup_{j=1}^{\min \{N+M-1, l\}} A_{n}^{\prime}(j)$. Let also $\hat{A}_{n}(l)=\sigma_{l}\left(\left\{Z_{n} \in\right.\right.$ $\left.\left.\Gamma, A_{n}(l)\right\}\right)$ and $\tilde{A}_{n}(l)=\left\{Z_{n} \in \Gamma, \bar{A}_{n}(l)\right\}$. As the average $Z_{n}$ is independent of the order of observations $\left\{X_{1}, \ldots, X_{n}\right\}$,

$$
\hat{A}_{n}(l) \subset \tilde{A}_{n}(l) \text { and }\left\{Z_{n} \in \Gamma, A_{n}(l)\right\}=\sigma_{l}^{-1} \sigma_{l}\left(Z_{n} \in \Gamma, A_{n}(l)\right) .
$$

Then, we may write

$$
\begin{aligned}
\nu_{x_{0}}\left(Z_{n} \in \Gamma, A_{n}(l)\right) & =\nu_{x_{0}}\left(\sigma_{l}^{-1}\left(\sigma_{l}\left(Z_{n} \in \Gamma, A_{n}(l)\right)\right)\right) \\
& =\nu_{x_{0}}\left(\vec{X}_{n} \in \cup_{\vec{x}_{n} \in \hat{A}_{n}} \sigma_{l}^{-1}\left(\vec{x}_{n}\right)\right) \\
& \leq \sum_{\vec{x}_{n} \in \hat{A}_{n}} \nu_{x_{0}}\left(\vec{X}_{n} \in \sigma_{l}^{-1}\left(\vec{x}_{n}\right)\right) \\
& \leq e^{o(n)} \sum_{\vec{x}_{n} \in \hat{A}_{n}} \hat{\mu}_{x_{0}}\left(\vec{X}_{n}=\vec{x}_{n}\right) \\
& \leq e^{o(n)} \sum_{\vec{x}_{n} \in \tilde{A}_{n}} \hat{\mu}_{x_{0}}\left(\vec{X}_{n}=\vec{x}_{n}\right)=e^{o(n)} \hat{\mu}_{x_{0}}\left(Z_{n} \in \Gamma, \bar{A}_{n}(l)\right) .
\end{aligned}
$$


Step 8. As $\cup_{l \geq 1} \bar{A}_{n}(l) \cup A_{n}(0) \subset\left\{\vec{X}_{n}\right.$ enters each $C_{i}$ at most once $\}$, we have

$$
\begin{aligned}
& \sum_{i=0}^{n-1} \nu_{x_{0}}\left(Z_{n} \in \Gamma, A_{n}(i)\right) \\
& \quad \leq\left(\left(1+(n-1) e^{o(n)}\right) \hat{\mu}_{x_{0}}\left(Z_{n} \in \Gamma, \vec{X}_{n} \text { enters each } C_{i} \text { at most once }\right) .\right.
\end{aligned}
$$

Then, noting (5.1), (5.2) and (5.11), we have

$\varlimsup \frac{1}{n} \log \mathbb{P}_{\pi}\left(Z_{n} \in \Gamma\right) \leq \max _{\substack{x_{0} \in \Sigma \\ \pi\left(x_{0}\right)>0}} \varlimsup \frac{1}{\lim } \frac{1}{n} \log \hat{\mu}_{x_{0}}\left(Z_{n} \in \Gamma, \vec{X}_{n}\right.$ enters each $C_{i}$ at most once).

Applying lemma 5.1 finishes the proof.

\section{$6 \quad$ Path Surgery Lower Bound}

The lower bound strategy is informed by the upper bound result. Namely, given the rearranged paths focused upon in the upper bound surgery, we can more or less restrict to them and gain lower bounds.

Proof of Proposition 4.7. When $N+M=1, P$ is irreducible, $C_{1}=\Sigma$ and $\mathcal{D}=\emptyset$. Then, $\check{\mathcal{P}}_{n}=P_{n}$ for all $n \geq 1$, and so $\mathbb{P}_{\pi}\left(Z_{n} \in \Gamma\right)=\check{\mu}_{\pi}\left(Z_{n} \in \Gamma\right)$. Also, as in the upper bound, $\vec{X}_{n}$ does not switch in this case. Hence, the lower bound holds trivially.

We will now assume that $N+M \geq 2$. Consider the subset $B \subset \Sigma^{n}$ formed from the following procedure.

1. For $1 \leq m \leq N+M$, let $J_{1}, J_{2}, \ldots, J_{m}$ be subpaths belonging respectively to distinct sets $C_{i_{1}}, C_{i_{2}}, \ldots, C_{i_{m}}$ where $\left\{i_{1}, \ldots, i_{m}\right\} \subset \mathcal{G}$. Let $j_{i}=\left|J_{i}\right|, J_{i}=\left\langle y_{1}^{i}, \ldots, y_{j_{i}}^{i}\right\rangle$ and $J_{i, 2}=\left\langle y_{2}^{i}, \ldots, y_{j_{i}}^{i}\right\rangle$ when $\left|j_{i}\right| \geq 2$ for $1 \leq i \leq m$. We impose now that the lengths satisfy $\sum_{i=1}^{m} j_{i}=n-E(N, M)$.

2. When $m \geq 2$, we connect subpaths $J_{s}$ and $J_{s+1}$ for $s=1, \ldots, m-1$ as follows. Let $0 \leq k \leq N+M-2$ be the number of sets entered in the connection, and let $L_{k}$ with $i=i_{s}$ and $j=i_{s+1}, Q_{k}$ and $V_{k}=\left\{\vec{x}^{s, 0}, \ldots, \vec{x}^{s, k+1}\right\}$ be as near (2.9). Denote $\vec{w}^{s}=\left\langle\vec{x}^{s, 0}, \ldots, \vec{x}^{s, k+1}\right\rangle$ and $k_{s}=\left|\vec{w}^{s}\right|$. Also, denote $b(s)=j_{s}+\sum_{i=1}^{s-1}\left(j_{i}+k_{i}\right)$. Let now $\vec{w}^{s}$ be such that

$$
\mathbb{P}_{\left(b(s), y_{2}^{s}\right)}\left(\vec{X}_{b(s)+1}^{b(s)+k_{s}+1}=\left\langle\vec{w}^{s}, y_{1}^{s+1}\right\rangle\right)=\underline{\gamma}^{1}\left(b(s)+1, y_{j_{s}}^{s}, y_{1}^{s+1}\right) .
$$

Then, in particular, as $\sum_{i=1}^{s-1} k_{i} \leq E(N, M)$, we have

$$
\begin{aligned}
\mathbb{P}_{\left(b(s), y_{2}^{s}\right)}\left(\vec{X}_{b(s)+1}^{b(s)+k_{s}+1}=\left\langle\vec{w}^{s}, y_{1}^{s+1}\right\rangle\right) & \geq \underline{\gamma}^{1}\left(b(s)+1,\left(i_{s}, i_{s+1}\right)\right) \\
& \geq \check{\gamma}^{0}\left(\sum_{i=1}^{s} j_{i}+1,\left(i_{s}, i_{s+1}\right)\right) .
\end{aligned}
$$


3. For $m \geq 2$, as $\sum_{i=1}^{m-1} k_{i} \leq E(N, M)$, the length of the concatenation satisfies

$$
\begin{aligned}
L & =\left|\left\langle J_{1}, \vec{w}^{1}, J_{2}, \ldots, \vec{w}^{m-1}, J_{m}\right\rangle\right| \\
& =n-E(N, M)+\sum_{i=1}^{m-1} k_{i} \leq n .
\end{aligned}
$$

When $m=1$, the length $L=\left|\left\langle J_{1}\right\rangle\right|=n-E(N, M)$.

If now $L<n$, we then augment the last subpath $J_{m}$ by $n-L \leq E(N, M)$ states in $C_{i_{m}}$. Specifically, define

$$
J_{m}^{\prime}=\left\{\begin{aligned}
J_{m} & \text { if } L=n \\
\left\langle J_{m}, x_{1}^{m}, \ldots, x_{n-L}^{m}\right\rangle & \text { if } L<n
\end{aligned}\right.
$$

where $\left\langle y_{j_{m}}^{m}, x_{1}^{m}, \ldots, x_{n-L}^{m}\right\rangle$ is a sequence of $n-L+1$ elements in $C_{i_{m}}$ with positive weight. Let also $J_{m, 2}^{\prime}=J_{m, 2}$ when $L=n$ and $J_{m, 2}^{\prime}=\left\langle J_{m, 2}, x_{1}^{m}, \ldots, x_{n-L}^{m}\right\rangle$ otherwise.

Let now

$$
\vec{x}_{n}=\left\{\begin{array}{rr}
\left\langle J_{1}, \vec{w}^{1}, \ldots, \vec{w}^{m-1}, J_{m}^{\prime}\right\rangle & \text { when } m \geq 2 \\
\left\langle J_{1}^{\prime}\right\rangle & \text { when } m=1 .
\end{array}\right.
$$

Finally, we define $B$ as the set of all such sequences $\vec{x}_{n}$ possible.

Now write

$$
\begin{aligned}
& \mathbb{P}_{\pi}\left(Z_{n} \in \Gamma_{2}\right) \\
& \geq \mathbb{P}_{\pi}\left(Z_{n} \in \Gamma_{2}, \vec{X}_{n} \in B\right) \\
& =\sum_{\vec{x}_{n} \in\left\{Z_{n} \in \Gamma_{2}\right\} \cap B} \mathbb{P}_{\pi}\left(\vec{X}_{j_{1}}=J_{1}\right) \underline{\gamma}\left(j_{1}+1, y_{j_{1}}^{1}, y_{1}^{2}\right) \mathbb{P}_{\left(j_{1}+k_{1}+1, y_{1}^{2}\right)}\left(\vec{X}_{j_{1}+k_{1}+2}^{j_{1}+k_{1}+j_{2}}=J_{2,2}\right) \\
& \quad \cdots \underline{\gamma}\left(b(m-1)+1, y_{j_{m-1}}^{m-1}, y_{1}^{m}\right) \mathbb{P}_{\left(\sum_{1}^{m-1}\left(j_{i}+k_{i}\right)+1, y_{1}^{m}\right)}\left(\vec{X}_{\sum_{1}^{m-1}\left(j_{i}+k_{i}\right)+2}^{\sum^{m-1}\left(k_{j}+k_{i}\right)+n}=J_{m, 2}^{\prime}\right) \\
& \geq c(L) \check{\mu}_{\pi}\left(Z_{n-E(N, M)} \in \Gamma_{2, n}, \vec{X}_{n-E(N, M)} \text { only enters }\left\{C_{i}: i \in \mathcal{G}\right\}\right. \\
& \text { with at most one visit to each set })
\end{aligned}
$$

where

$$
c(L)=\left\{\begin{array}{rr}
\mathbb{P}_{\left(b(m), y_{j m}^{m}\right)}\left(\vec{X}_{b(m)+1}^{b(m)+n-L}=\left\langle x_{1}^{m}, \ldots, x_{n-L}^{m}\right\rangle\right) & \text { when } n>L \\
1 & \text { when } n=L
\end{array}\right.
$$

and

$$
\Gamma_{2, n}=\frac{n}{n-E(N, M)} B\left(x_{0}, a / 2-\frac{E(N, M)\|f\|}{n}\right) .
$$

In the last step, we rewrote $\mathbb{P}_{\pi}$ in terms of the measure $\check{\mu}_{\pi}$ by collapsing together the subpaths $\left\{J_{i}\right\}$. At the same time, since the collapsed path $\left\langle J_{1}, \ldots, J_{m}\right\rangle$ is of length $n-E(N, M)$, we correct the set $\Gamma_{2}$ to $\Gamma_{2, n}$. 
We now estimate the prefactor $c(L)$. With respect to the minimum probability $p_{\min }$ (cf. (2.5)), and $n>L$ large, as $P_{n} \rightarrow P$, we can certainly bound

$$
\mathbb{P}_{\left(b(m), y_{j m}^{m}\right)}\left(\vec{X}_{b(m)+1}^{b(m)+n-L}=\left\langle x_{1}^{m}, \ldots, x_{n-L}^{m}\right\rangle\right) \geq p_{\min }^{E(N, M)} / 2 .
$$

Therefore, $\lim (\log c(L)) / n=0$.

Hence, the proposition follows by taking "lim" in (6.1), and simple estimates.

\section{Homogeneous "Rest Cost" Replacement}

We replace certain apriori nonhomogeneous "resting" weights with homogeneous ones for both upper and lower bound estimates.

Proof of Propositions 4.3 and 4.8. Follows as direct corollaries of the more general Proposition 7.1 below.

Proposition 7.1 Let $\left\{B_{n}\right\} \subset \mathbb{R}^{d}$ be a a sequence of Borel sets.

(Upper Bound) For $\epsilon_{1}, \epsilon_{2}>0$, we have

$$
\varlimsup \frac{1}{n} \log \hat{\mu}_{\pi, \epsilon_{1}, \epsilon_{2}}\left(\vec{X}_{n} \in B_{n}\right) \leq \varlimsup \lim \frac{1}{n} \log \bar{\mu}_{\pi, \epsilon_{1}, \epsilon_{2}}\left(\vec{X}_{n} \in B_{n}\right) .
$$

(Lower Bound) Suppose $n_{0}\left(\left\{P_{n}\right\}\right)=1$. Then, we have

$$
\underline{\lim } \frac{1}{n} \log \check{\mu}_{\pi}\left(\vec{X}_{n} \in B_{n}\right) \geq \underline{\lim } \frac{1}{n} \log \underline{\mu}_{\pi}\left(\vec{X}_{n} \in B_{n}\right) .
$$

Proof. We will prove the "lower bound" part, as the "upper bound" estimate follows analogously and more simply. Let $G=\left\{(s, t): p(s, t)>0\right.$ where $s, t \in C_{i}$ for $\left.i \in \mathcal{G}\right\}$. As $P_{n} \rightarrow P$, the state space is finite, and by assumption $n_{0}=1$, there exists $\alpha>0$ and a sequence $\alpha \leq m(k) \uparrow 1$ such that $m(k) \leq p_{k}(s, t) / p(s, t)$ for all $(s, t) \in G$ and $k \geq 1$. Write now that

$$
\begin{aligned}
\check{\mu}_{\pi} & \left(\vec{X}_{n} \in B_{n}\right) \\
& =\sum_{x_{0} \in \Sigma} \sum_{\vec{x}_{n} \in B_{n}} \pi\left(x_{0}\right) \prod_{i=1}^{n} \check{p}_{i}\left(x_{i-1}, x_{i}\right) \\
& =\sum_{x_{0} \in \Sigma} \sum_{\vec{x}_{n} \in B_{n}} \pi\left(x_{0}\right) \prod_{\left(x_{i-1}, x_{i}\right) \in G^{c}} \check{p}_{i}\left(x_{i-1}, x_{i}\right) \prod_{\left(x_{i-1}, x_{i}\right) \in G} \frac{\check{p}_{i}\left(x_{i-1}, x_{i}\right)}{p\left(x_{i-1}, x_{i}\right)} p\left(x_{i-1}, x_{i}\right) \\
& \geq \sum_{x_{0} \in \Sigma} \sum_{\vec{x}_{n} \in B_{n}} \pi\left(x_{0}\right) \prod_{\left(x_{i-1}, x_{i}\right) \in G^{c}} \underline{p}_{i}\left(x_{i-1}, x_{i}\right) \prod_{\left(x_{i-1}, x_{i}\right) \in G} \frac{p_{i}\left(x_{i-1}, x_{i}\right)}{p\left(x_{i-1}, x_{i}\right)} \underline{p}_{i}\left(x_{i-1}, x_{i}\right) \\
& \geq\left[\prod_{i=1}^{n} m(i)\right] \sum_{x_{0} \in \Sigma} \sum_{\vec{x}_{n} \in B_{n}} \pi\left(x_{0}\right) \prod_{\left(x_{i-1}, x_{i}\right) \in G^{c}} \underline{p}_{i}\left(x_{i-1}, x_{i}\right) \prod_{\left(x_{i-1}, x_{i}\right) \in G} \underline{p}_{i}\left(x_{i-1}, x_{i}\right) \\
& =\left[\prod_{i=1}^{n} m(i)\right] \underline{\mu}_{\pi}\left(\vec{X}_{n} \in B_{n}\right) .
\end{aligned}
$$


Indeed, for the first bound, we note, if $\left(x_{i-1}, x_{i}\right) \notin G$, that $\check{p}_{i}\left(x_{i-1}, x_{i}\right)=p_{i}\left(x_{i-1}, x_{i}\right)$ when $\left(x_{i-1}, x_{i}\right)$ connects distinct sets in $\mathcal{G}$, and $\check{p}_{i}\left(x_{i-1}, x_{i}\right) \geq 0=\underline{p}_{i}\left(x_{i-1}, x_{i}\right)$ otherwise. The second bound follows by monotonicity of $\{m(i)\}$.

Then, the proposition lower bound follows as $\left(\sum_{1}^{n} \log m(i)\right) / n \rightarrow 0$.

\section{Upper Coarse Graining Bounds}

The plan will be to optimize over a coarse graining of the possible locations $Z_{n}$ visits in $\mathbb{K}$ and associated visit times. Some additional definitions which build on those in section 5 are required in this effort.

Define, for $1 \leq H \leq N+M$ and $\underline{i}_{H}=\left\langle i_{1}, \ldots, i_{H}\right\rangle$ composed of distinct indices in $\{1, \ldots, N+M\}$, that

$$
C\left(\underline{i}_{H}\right)=\left\{\vec{X}_{n} \text { starts in } C_{i_{1}} \text { and enters successively } C_{i_{2}}, \ldots, C_{i_{H}}\right\} .
$$

Also, let $k_{0}=0, k_{H}=n$, and when $H \geq 2$ let $1 \leq k_{1}<\cdots<k_{H-1} \leq n-1$ and denote $\vec{k}_{H}=\left\langle k_{0}, \ldots, k_{H}\right\rangle$ and

$$
S_{n}\left(\vec{k}_{H}\right)=\left\{\vec{X}_{n} \text { switches at times } k_{1}, k_{2}, \ldots, k_{H-1}\right\} .
$$

Let also

$$
\vec{v}_{\vec{k}_{H}}=\left\langle k_{1} / n,\left(k_{2}-k_{1}\right) / n, \ldots,\left(n-k_{H-1}\right) / n\right\rangle .
$$

We now specify a certain cube decomposition. For $\vec{v} \in \Omega_{H}$ and $z \in \mathbb{R}^{d}$, recall the set $D(H, \vec{v}, z)$ (cf. $(2.6))$ and let $D(H, \vec{v}, B)=\cup_{z \in B} D(H, \vec{v}, z)$ for sets $B \subset \mathbb{R}^{d}$.

Let now $F_{1}$ be the regular partition of $\mathbb{K}$ into $2^{d}$ closed cubes, $\left\{\Delta_{s}^{1}: 1 \leq s \leq 2^{d}\right\}$, whose interiors nonintersect and $\cup_{s} \Delta_{s}^{1}=\mathbb{K}$. For $n \geq 2$, let also $F_{n}$ be the regular refinement of $F_{n-1}$ into $2^{n-1}\left(2^{d}\right)$ closed cubes, $\left\{\Delta_{s}^{n}: 1 \leq s \leq 2^{n-1}\left(2^{d}\right)\right\}$, where also $\cup_{s} \Delta_{s}^{n}=\mathbb{K}$. Observe also that the $\left(2^{n-1}\left(2^{d}\right)\right)^{H}$ subcubes formed from $F_{n},\{\Delta(n, \vec{s})=$ $\left.\Delta_{s_{1}}^{n} \times \cdots \times \Delta_{s_{H}}^{n}: 1 \leq s_{i} \leq 2^{n-1}\left(2^{d}\right)\right\}$, refine $\mathbb{K}^{H}$ as well.

For $B \subset \mathbb{K}$ and $j \geq 1$, define

$$
D_{j}(H, \vec{v}, B)=\cup\{\Delta(j, \vec{s}): \Delta(j, \vec{s}) \cap D(H, \vec{v}, B) \neq \emptyset\}
$$

be the non-empty union of all subcubes with respect to $j$ th partition which intersect $D(H, \vec{v}, B)$. Let also

$$
F(H, n, \vec{v}, B)=\left\{\vec{s}: \Delta(n, \vec{s}) \subset D_{n}(H, \vec{v}, B)\right\}
$$

For $\alpha>0$, let $m_{\alpha}$ be the first partition level $m$ so that, for each $1 \leq l \leq N+M$, $\left|\mathbb{I}_{l, \epsilon_{1}, \epsilon_{2}}(x)-\mathbb{I}_{l, \epsilon_{1}, \epsilon_{2}}(y)\right| \leq \alpha$ when $|x-y| \leq \operatorname{diam}(\Delta(m, \cdot))$ and $x, y \in Q_{l, \epsilon_{1}, \epsilon_{2}}$.

We will also need the following technical lemmas which can be skipped on first reading. 
Lemma 8.1 For distinct $i, j \in \mathcal{G}$, we have

$$
\mathcal{U}_{0}(i, j)=\varlimsup \frac{1}{n} \log \gamma(n,(i, j)) .
$$

Proof. Write the left side as

$$
\begin{aligned}
& \varlimsup \frac{1}{n} \log \gamma(n,(i, j))=\varlimsup_{0 \leq k \leq M-2} \max _{L_{k}} \sum_{s=0}^{k} \frac{1}{n} \log \hat{t}\left(n+s,\left(l_{s}, l_{s+1}\right)\right) \\
& =\max _{0 \leq k \leq M-2} \max _{L_{k}} \sum_{s=0}^{k} \lim \frac{1}{n} \log \hat{t}\left(n,\left(l_{s}, l_{s+1}\right)\right) \\
& =\max _{0 \leq k \leq M-2} \max _{L_{k}} \sum_{s=0}^{k} v\left(l_{s}, l_{s+1}\right)=\mathcal{U}_{0}(i, j)
\end{aligned}
$$

where the second and third lines follow since the $\operatorname{limit} \lim \log \hat{t}(n,(k, l)) / n=v(k, l)$ holds from Lemma 4.1.

In the next result, let $1 \leq H \leq N+M$ and $\Gamma \subset \mathbb{K}$ be a closed set. Let also $\mathbb{I}_{l}^{\theta}=\min \left\{\mathbb{I}_{l, \epsilon_{1}, \epsilon_{2}}, \theta\right\}$ for $\theta \geq 1$ and $1 \leq l \leq N+M$.

Lemma 8.2 Let $\vec{v}^{n} \in \Omega_{H}$ be a convergent sequence, $\lim _{n} \vec{v}^{n}=\vec{v} \in \Omega_{H}$. Then, for any $\underline{i}_{H}$, we have

$$
\varlimsup_{\theta \uparrow \infty} \varlimsup_{m \uparrow \infty} \varlimsup_{n \rightarrow \infty} \inf _{\vec{x} \in D_{m}\left(H, \vec{v}^{n}, \Gamma\right)} \sum_{j=1}^{H} v_{j}^{n} \mathbb{I}_{i_{j}}^{\theta}\left(x_{j}\right) \geq \inf _{\vec{x} \in D(H, \vec{v}, \Gamma) \cap \mathbb{K}} \sum_{j=1}^{H} v_{j} \mathbb{I}_{i_{j}, \epsilon_{1}, \epsilon_{2}}\left(x_{j}\right) .
$$

Proof. As $D_{m}\left(H, \vec{v}^{n}, \Gamma\right) \subset \mathbb{K}^{H}$, and $\mathbb{K}^{H}$ is compact, we can find a convergent sequence $\vec{x}^{m, n_{k}} \in D_{m}\left(H, \vec{v}^{n_{k}}, \Gamma\right) \rightarrow \vec{x}^{m} \in \mathbb{K}^{H}$ so that by lower semicontinuity of $\left\{\mathbb{I}_{l}^{\theta}\right\}$

$$
\begin{aligned}
\varlimsup_{n \rightarrow \infty} \inf _{\vec{x} \in D_{m}\left(H, \vec{v}^{n}, \Gamma\right)} \sum_{j=1}^{H} v_{j}^{n} \mathbb{I}_{i_{j}}^{\theta}\left(x_{j}\right) & =\lim _{k \rightarrow \infty} \sum_{j=1}^{H} v_{j}^{n_{k}} \mathbb{I}_{i_{j}}^{\theta}\left(x_{j}^{m, n_{k}}\right) \\
& \geq \sum_{j=1}^{H} v_{j} \mathbb{I}_{i_{j}}^{\theta}\left(x_{j}^{m}\right) .
\end{aligned}
$$

Now, out of $\left\{\vec{x}^{m}\right\} \subset \mathbb{K}^{H}$, let $\vec{x}^{m_{j}} \rightarrow \vec{x} \in \mathbb{K}^{H}$ be a convergent subsequence on which $\varlimsup_{m \uparrow \infty} \sum_{j=1}^{H} v_{j} \mathbb{I}_{i_{j}}^{\theta}\left(x_{j}^{m}\right)$ is attained. Also observe that $\mathbb{I}_{l}^{\theta}\left(x_{l}\right) \rightarrow \mathbb{I}_{l, \epsilon_{1}, \epsilon_{2}}\left(x_{l}\right)$ for $1 \leq l \leq N+M$ as $\theta \uparrow \infty$. Then, again by lower semicontinuity,

$$
\varlimsup_{\theta \uparrow \infty} \varlimsup_{m \uparrow \infty} \varlimsup_{n \rightarrow \infty} \inf _{n \rightarrow D_{m}\left(H, \vec{v}^{n}, \Gamma\right)} \sum_{j=1}^{H} v_{j}^{n} \mathbb{I}_{i_{j}}^{\theta}\left(x_{j}\right) \geq \varlimsup_{\theta \uparrow \infty} \sum_{j=1}^{H} v_{j} \mathbb{I}_{i_{j}}^{\theta}\left(x_{j}\right)=\sum_{j=1}^{H} v_{j} \mathbb{I}_{i_{j}, \epsilon_{1}, \epsilon_{2}}\left(x_{j}\right) .
$$


To finish the argument, we show that $\vec{x} \in D(H, \vec{v}, \Gamma) \cap \mathbb{K}^{H}$. By construction, the diameters of the partitioning cubes $\Delta(m, \cdot)$ uniformly vanish as $m \uparrow \infty$. As $D_{m}\left(H, \vec{v}^{n_{k}}, \Gamma\right)$ is composed of cubes which intersect $D\left(H, \vec{v}^{n_{k}}, \Gamma\right)$, we have that any point in $D_{m}\left(H, \vec{v}^{n_{k}}, \Gamma\right)$ is at most a distance $\operatorname{diam}(\Delta(m, \cdot))$ away from $D\left(H, \vec{v}^{n_{k}}, \Gamma\right) \cap$ $\mathbb{K}^{H}$. Hence, there are points $\vec{y}^{m, n_{k}} \in D\left(H, \vec{v}^{n_{k}}, \Gamma\right) \cap \mathbb{K}^{H}$ such that $\left|\vec{x}^{m, n_{k}}-\vec{y}^{m, n_{k}}\right| \leq$ $\operatorname{diam}(\Delta(m, \cdot))$. Let $\vec{y}^{m, n_{k}^{\prime}} \rightarrow \vec{y}^{m} \in \mathbb{K}^{H}$ be a convergent subsequence. We have then $\left|\vec{x}^{m}-\vec{y}^{m}\right| \leq \operatorname{diam}(\Delta(m, \cdot))$. Now since $\Gamma$ is closed, and $\sum_{j=1}^{H} v_{j}^{n_{k}^{\prime}} y_{j}^{m, n_{k}^{\prime}} \in \Gamma$ for all $m, k$, we have

$$
\lim _{m} \lim _{k} \sum_{j=1}^{H} v_{j}^{n_{k}^{\prime}} y_{j}^{m, n_{k}^{\prime}}=\lim _{m} \sum_{j=1}^{H} v_{j} y_{j}^{m}=\sum_{j=1}^{H} v_{j} x_{j} \in \Gamma
$$

and so $\vec{x} \in D(H, \vec{v}, \Gamma) \cap \mathbb{K}^{H}$.

Proof of Proposition 4.4. When $N+M=1$, there is only one irreducible subset $C_{1}=\Sigma$ and $\overline{\mathcal{P}}_{k, \epsilon_{1}, \epsilon_{2}}=P\left(1, \epsilon_{1}\right)$ for $k \geq 2$. So, modulo a first transition (with respect to the constant matrix $\overline{\mathcal{P}}_{1}$ ), the measure $\bar{\mu}_{\pi}$ is a "homogeneous non-negative process" with respect to $P\left(1, \epsilon_{1}\right)$. Also, as there can no "repeat visits," and $\mathbb{J}_{\mathcal{U}_{0}, \epsilon_{1}, \epsilon_{2}}=\mathbb{I}_{1, \epsilon_{1}, \epsilon_{2}}$ in this case, the proposition follows from the LDP in Proposition 2.1.

We will now assume that $N+M \geq 2$. Also, to reduce notation we will suppress subscripts $\epsilon_{1}$ and $\epsilon_{2}$ when there is no confusion in the following.

Step 1. As $Z_{n}$ takes only values in the set $\mathbb{K}$ we have

$$
\begin{aligned}
& \bar{\mu}_{\pi, \epsilon_{1}, \epsilon_{2}}\left(Z_{n} \in \bar{\Gamma}, \vec{X}_{n} \text { enters each } C_{i} \text { at most once }\right) \\
& =\sum_{1 \leq H \leq N+M} \sum_{\underline{i}_{H}} \bar{\mu}_{\pi, \epsilon_{1}, \epsilon_{2}}\left(Z_{n} \in \bar{\Gamma} \cap \mathbb{K}, A_{n}^{\prime}(H-1), C\left(\underline{i}_{H}\right)\right)
\end{aligned}
$$

where the sum on $\underline{i}_{H}$ is over $\left(\begin{array}{c}N+M \\ H\end{array}\right) H$ ! possibilities.

Step 2. We first consider the case when "switching" actually occurs. Let $2 \leq H \leq$ $N+M$ and fix indices $\underline{i}_{H}$. Write, for $n>N+M$ (larger than the number of switches), that

$\bar{\mu}_{\pi}\left(Z_{n} \in \bar{\Gamma} \cap \mathbb{K}, A_{n}^{\prime}(H-1), C\left(\underline{i}_{H}\right)\right)=\sum_{\vec{k}_{H}} \bar{\mu}_{\pi}\left(Z_{n} \in \bar{\Gamma} \cap \mathbb{K}, A_{n}^{\prime}(H-1), C\left(\underline{i}_{H}\right), S_{n}\left(\vec{k}_{H}\right)\right)$

where the sum on $\vec{k}_{H}$ comprises $\left(\begin{array}{c}n-1 \\ H-1\end{array}\right)$ possibilities.

For convenience, denote $\bar{B}=\bar{\Gamma} \cap \mathbb{K}$ and

$$
E_{n}=A_{n}^{\prime}(H-1) \cap C\left(\underline{i}_{H}\right) \cap S_{n}\left(\vec{k}_{H}\right) .
$$

Let also $\alpha>0$, and let $m \geq m_{\alpha}$. Recall from part "LDP for Hom ..." in section 2, 
that $Z_{i}^{j} \in \mathbb{K}$ for $i \leq j$, and so we may write the summand in (8.2) equal to

$$
\begin{aligned}
\bar{\mu}_{\pi}\left(\left\langle Z_{1}^{k_{1}}, \ldots, Z_{k_{H-1}+1}^{n}\right\rangle \in D\left(H, \vec{v}_{\vec{k}_{H}}, \bar{B}\right) \cap \mathbb{K}^{H}, E_{n}\right) \\
\leq \bar{\mu}_{\pi}\left(\left\langle Z_{1}^{k_{1}}, \ldots, Z_{k_{H-1}+1}^{n}\right\rangle \in D_{m}\left(H, \vec{v}_{\vec{k}_{H}}, \bar{B}\right), E_{n}\right) \\
=\bar{\mu}_{\pi}\left(\left\langle Z_{1}^{k_{1}}, \ldots, Z_{k_{H-1}+1}^{n}\right\rangle \in \cup_{\vec{s}} \Delta(m, \vec{s}), E_{n}\right) \\
\leq \sum_{\vec{s}} \bar{\mu}_{\pi}\left(Z_{1}^{k_{1}} \in \Delta_{s_{1}}^{m}, \ldots, Z_{k_{H-1}+1}^{n} \in \Delta_{s_{H}}^{m}, E_{n}\right)
\end{aligned}
$$

where the union and sum is over $\vec{s} \in F\left(H, m, \vec{v}_{\vec{k}_{H}}, \bar{B}\right)$

Step 3. For $1 \leq l \leq N+M$, let $\pi_{l}$ be the uniform distribution on $C_{l}$, and let $\mathbb{P}_{\pi, \epsilon_{1}, \epsilon_{2}}^{l}$ denote the homogeneous non-negative measure on $C_{l}$ formed from Construction $(\mathrm{CON})$ with $U_{n} \equiv P\left(l, \epsilon_{1}, \epsilon_{2}\right)$ and initial distribution $\pi$. Let also $\theta>$ $\max _{1 \leq l \leq N+M} \max _{x \in Q_{l, \epsilon_{1}, \epsilon_{2}}} \mathbb{I}_{l, \epsilon_{1}, \epsilon_{2}}(x)$ be a number larger than the maxima of the rate functions on their domains of finiteness (cf. Proposition 2.1).

We now use the Markov property (2.2), and simple estimates, to further bound the summand in (8.3) as

$$
\begin{aligned}
& \bar{\mu}_{\pi}\left(Z_{1}^{k_{1}} \in \Delta_{s_{1}}^{m}, \ldots, Z_{k_{H-1}+1}^{n} \in \Delta_{s_{H}}^{m}, E_{n}\right) \\
& \leq \bar{\mu}_{\pi}\left(Z_{1}^{k_{1}} \in \Delta_{s_{1}}^{m}, \vec{X}_{1}^{k_{1}} \text { in } C_{i_{1}}\right) \\
& \quad \cdot \prod_{j=1}^{H-1}\left|C_{i_{j}}\right| \gamma\left(k_{j}+1,\left(i_{j}, i_{j+1}\right)\right) \bar{\mu}_{\left(\pi_{i_{j+1}}, k_{j}+1\right)}\left(Z_{k_{j}+1}^{k_{j+1}} \in \Delta_{s_{j+1}}^{m}, \vec{X}_{k_{j}+1}^{k_{j+1}} \text { in } C_{i_{j+1}}\right) \\
& \leq \prod_{j=1}^{H-1} \gamma\left(k_{j}+1,\left(i_{j}, i_{j+1}\right)\right) \prod_{j=0}^{H-1}\left|C_{i_{j+1}}\right| \mathbb{P}_{\left(\pi_{i_{j+1}}, k_{j}+1\right)}^{i_{j+1}}\left(Z_{k_{j}+1}^{k_{j+1}} \in \Delta_{s_{j+1}}^{m}\right) .
\end{aligned}
$$

Step 4. Recall the definition of $\mathbb{I}_{l}^{\theta}$ just before Lemma 8.2. Let

$$
c\left(k_{j+1}-k_{j} ; \Delta_{s_{j+1}}^{m}, \theta, C_{i_{j+1}}\right)=\mathbb{P}_{\left(\pi_{i_{j+1}}, k_{j}+1\right)}^{i_{j+1}}\left(Z_{k_{j}+1}^{k_{j+1}} \in \Delta_{s_{j+1}}^{m}\right) e^{\left(k_{j+1}-k_{j}\right) \mathbb{I}_{i_{j+1}}^{\theta}\left(\Delta_{s_{j+1}}^{m}\right)} .
$$

From homogeneous non-negative large deviation upper bounds (cf. Proposition 2.1), uniformly over $H, \vec{k}_{H}$, the finite number of cubes $\vec{s}$ at level $m$, and $\underline{i}_{H}$, we have $c\left(k_{j+1}-k_{j} ; \Delta_{s_{j+1}}^{m}, \theta, C_{i_{j+1}}\right) \leq e^{o(n)}$.

Also by monotonicity $\gamma(i+1, \ldots) \leq \gamma(i, \ldots)$, Then, we have (8.4) is less than

$$
e^{o(n)}\left[\prod_{j=1}^{H-1} \gamma\left(k_{j},\left(i_{j}, i_{j+1}\right)\right)\right] \exp \left\{-\sum_{j=1}^{H}\left(k_{j}-k_{j-1}\right) \mathbb{I}_{i_{j}}^{\theta}\left(\Delta_{s_{j}}^{m}\right)\right\} .
$$

Step 5. At this point, we now bound the terms corresponding to no "switching" in (8.1), that is when $H=1$. For $1 \leq i_{1} \leq N+M$, we have

$$
\bar{\mu}_{\pi}\left(Z_{n} \in \bar{\Gamma} \cap \mathbb{K}, A_{n}^{\prime}(0), C\left(i_{1}\right)\right) \leq e^{o(n)} \sum_{s_{1} \in F(1, n, 1, \bar{B})} \exp \left\{-n \mathbb{I}_{i_{1}}^{\theta}\left(\Delta_{s_{1}}^{m}\right)\right\}
$$


Step 6. It will be convenient to define now $\gamma\left(0,\left(l, l^{\prime}\right)\right)=1$ for distinct $1 \leq l, l^{\prime} \leq$ $N+M$. We now combine (8.5) and (8.6) to bound (8.1) as

$$
\begin{aligned}
\bar{\mu}_{\pi}\left(Z_{n} \in \bar{\Gamma}, \vec{X}_{n} \text { enters each set } C_{i} \text { at most once }\right) & \sum_{1 \leq H \leq N+M} \sum_{\underline{i}_{H}} \sum_{\vec{k}_{H}} \sum_{\vec{s}} \\
& \quad\left[\prod_{j=1}^{H} \gamma\left(k_{j-1},\left(i_{j-1}, i_{j}\right)\right)\right] \exp \left\{-\sum_{j=1}^{H}\left(k_{j}-k_{j-1}\right) \mathbb{I}_{i_{j}}^{\theta}\left(\Delta_{s_{j}}^{m}\right)\right\} .
\end{aligned}
$$

[Note that (8.6) corresponds to index $H=1$.]

Since the sum over $\vec{s} \in F\left(H, m, \vec{v}_{\vec{k}_{H}}, \bar{B}\right)$ contains at most $\left(2^{m-1}\left(2^{d}\right)\right)^{H}$ terms, we can apply Lemma 5.1 to obtain

$$
\begin{aligned}
& \varlimsup \frac{1}{n} \log \bar{\mu}_{\pi}\left(Z_{n} \in \bar{\Gamma}, \vec{X}_{n} \text { enters each set } C_{i}\right. \text { at most once) } \\
& \leq \varlimsup_{1 \leq H \leq N+M} \max _{\underline{i}_{H}} \max _{\vec{k}_{H}} \max _{\vec{s}} \\
& \sum_{j=0}^{H-1} \frac{1}{n} \log \gamma\left(k_{j},\left(i_{j}, i_{j+1}\right)\right)-\sum_{j=1}^{H} \frac{\left(k_{j}-k_{j-1}\right)}{n} \mathbb{I}_{i_{j}}^{\theta}\left(\Delta_{s_{j}}^{m}\right) \text {. }
\end{aligned}
$$

Step 7. Now, by the choice of $\theta$, we have $\mathbb{I}_{l}^{\theta}=\mathbb{I}_{l}$ on $Q_{l}$ for $1 \leq l \leq N+M$. Also, recall that $\mathbb{I}_{l}$ is uniformly continuous on $Q_{l}$ for $1 \leq l \leq N+M$ (Proposition 2.1). Then, for $\vec{s} \in F\left(H, m, \vec{v}_{\vec{k}_{H}}, \bar{B}\right)$ such that $\Delta(m, \vec{s}) \cap Q_{i_{1}} \times \cdots \times Q_{i_{H}} \neq \emptyset$, we have

$$
\begin{aligned}
\frac{1}{n} \sum_{j=1}^{H}\left(k_{j}-k_{j-1}\right) \mathbb{I}_{i_{j}}^{\theta}\left(\Delta_{s_{j}}^{m}\right) & =\frac{1}{n} \sum_{j=1}^{H}\left(k_{j}-k_{j-1}\right) \inf _{x_{j} \in \Delta_{s_{j}}^{m} \cap Q_{j}} \mathbb{I}_{i_{j}}\left(x_{j}\right) \\
& \geq \inf _{\vec{x} \in \Delta(m, \vec{s}) \cap \prod_{l=1}^{H} Q_{i_{l}}} \frac{1}{n} \sum_{j=1}^{H}\left(k_{j}-k_{j-1}\right) \mathbb{I}_{i_{j}}\left(x_{j}\right)-\alpha \\
& =\inf _{\vec{x} \in \Delta(m, \vec{s}) \cap \prod_{l=1}^{H} Q_{i_{l}}} \frac{1}{n} \sum_{j=1}^{H}\left(k_{j}-k_{j-1}\right) \mathbb{I}_{i_{j}}^{\theta}\left(x_{j}\right)-\alpha \\
& \geq \inf _{\vec{x} \in \Delta(m, \vec{s})} \frac{1}{n} \sum_{j=1}^{H}\left(k_{j}-k_{j-1}\right) \mathbb{I}_{i_{j}}^{\theta}\left(x_{j}\right)-\alpha .
\end{aligned}
$$

On the other hand, if there exists $G \subset\{1, \ldots, H\}$ such that $\Delta_{s_{j}}^{m} \cap Q_{i_{j}}=\emptyset$ for all 
$j \in G$, we have that $\mathbb{I}_{i_{j}}^{\theta}\left(\Delta_{s_{j}}^{m}\right)=\inf _{x_{j} \in \Delta_{s_{j}}} \mathbb{I}_{i_{j}}=\theta$. Then, combining with (8.8), we have

$$
\begin{aligned}
\frac{1}{n} \sum_{j=1}^{H}\left(k_{j}-k_{j-1}\right) \mathbb{I}_{i_{j}}^{\theta}\left(\Delta_{s_{j}}^{m}\right) & =\frac{1}{n} \sum_{j \in G^{c}}\left(k_{j}-k_{j-1}\right) \mathbb{I}_{i_{j}}^{\theta}\left(\Delta_{s_{j}}^{m}\right)+\frac{1}{n} \sum_{j \in G}\left(k_{j}-k_{j-1}\right) \mathbb{I}_{i_{j}}^{\theta}\left(\Delta_{s_{j}}^{m}\right) \\
& \geq \inf _{\vec{x} \in \Delta(m, \vec{s})} \frac{1}{n} \sum_{j=1}^{H}\left(k_{j}-k_{j-1}\right) \mathbb{I}_{i_{j}}^{\theta}\left(x_{j}\right)-\alpha .
\end{aligned}
$$

With the estimate (8.9), we have (8.7) is less than

$$
\begin{aligned}
\varlimsup & \max _{H} \max _{\underline{i}_{H}} \max _{\vec{k}_{H}} \sum_{j=0}^{H-1} \frac{1}{n} \log \gamma\left(k_{j},\left(i_{j}, i_{j+1}\right)\right) \\
& -\inf _{\vec{x} \in D_{m}\left(H, \vec{v}_{\vec{k}_{H}}, \bar{B}\right)} \frac{1}{n} \sum_{j=1}^{H}\left(k_{j}-k_{j-1}\right) \mathbb{I}_{i_{j}}^{\theta}\left(x_{j}\right)+\alpha .
\end{aligned}
$$

Step 8. Without loss of generality, we may assume that the $\varlimsup$ lim sequence in (8.10) occurs on a subsequence with fixed $1 \leq H \leq N+M$ and $\underline{i}_{H}$, and vectors $\vec{k}_{H}^{n}$ where

$$
\vec{v}_{\vec{k}_{H}^{n}}=\vec{v}^{n} \rightarrow \vec{v}=\left\langle v_{1}, \ldots, v_{H}\right\rangle
$$

and

$$
\lim _{n \rightarrow \infty} \frac{1}{n} \log \gamma\left(k_{j}^{n},\left(i_{j}, i_{j+1}\right)\right) \text { exists for } 1 \leq j \leq H .
$$

As values of $\theta$ and $m$ above a certain range are arbitrary, by Lemma 8.2, we have

$$
\varlimsup_{\theta \uparrow \infty} \varlimsup_{m \uparrow \infty} \lim _{n \rightarrow \infty} \inf _{\vec{x} \in D_{m}\left(H, \vec{v}^{n}, \bar{B}\right)} \sum_{j=1}^{H} v_{j}^{n} \mathbb{I}_{i_{j}}^{\theta}\left(x_{j}\right) \geq \inf _{\vec{x} \in D(H, \vec{v}, \bar{B}) \cap \mathbb{K}^{H}} \sum_{j=1}^{H} v_{j} \mathbb{I}_{i_{j}, \epsilon_{1}, \epsilon_{2}}\left(x_{j}\right) .
$$

Step 9. We now argue that

$$
\lim \frac{1}{n} \log \gamma\left(k_{j}^{n},\left(i_{j}, i_{j+1}\right)\right) \leq\left(\sum_{l=1}^{j} v_{l}\right) \varlimsup \frac{1}{n} \log \gamma\left(n,\left(i_{j}, i_{j+1}\right)\right) .
$$

Indeed, by definition $\sum_{l=1}^{j} v_{l}=\lim k_{j}^{n} / n$ for $1 \leq j \leq N+M$. Then, as

$$
\frac{1}{n} \log \gamma\left(k_{j}^{n},\left(i_{j}, i_{j+1}\right)\right)=\frac{k_{j}^{n}}{n} \frac{1}{k_{j}^{n}} \log \gamma\left(k_{j}^{n},\left(i_{j}, i_{j+1}\right)\right)
$$

the inequality (8.11) follows easily when $\sum_{l=1}^{l} v_{l}>0$ or $0 \geq \varlimsup\left(\lim \left(\log \gamma\left(k_{j}^{n},\left(i_{j}, i_{j+1}\right)\right)\right) / n>\right.$ $-\infty$. But, also in the exceptional case (8.11) still holds: As $\log \gamma\left(k_{j}^{n},\left(i_{j}, i_{j+1}\right)\right) \leq 0$, we have by the convention $0 \cdot(-\infty)=0$ that

$$
\lim \frac{1}{n} \log \gamma\left(k_{j}^{n},\left(i_{j}, i_{j+1}\right)\right) \leq 0=0 \cdot(-\infty)=\left(\sum_{l=1}^{j} v_{l}\right) \varlimsup \frac{1}{n} \log \gamma\left(n,\left(i_{j}, i_{j+1}\right)\right) .
$$


Therefore, we have

$$
\begin{aligned}
\sum_{j=0}^{H-1} \lim \frac{1}{n} \log \gamma\left(k_{j}^{n},\left(i_{j}, i_{j+1}\right)\right) & \leq 1_{[H \geq 2]} \sum_{j=0}^{H-1}\left(\sum_{l=1}^{j} v_{l}\right) \varlimsup \frac{1}{n} \log \gamma\left(n,\left(i_{j}, i_{j+1}\right)\right) \\
& =1_{[H \geq 2]} \sum_{j=1}^{H-1}\left(\sum_{l=1}^{j} v_{l}\right) \mathcal{U}_{0}\left(i_{j}, i_{j+1}\right)
\end{aligned}
$$

from Lemma 8.1 where the indicator reflects that the right-side vanishes when $H=1$. So, (8.10) is bounded above by

$$
\begin{gathered}
1_{[H \geq 2]} \sum_{j=0}^{H-1}\left(\sum_{l=1}^{j} v_{l}\right) \mathcal{U}_{0}\left(i_{j}, i_{j+1}\right)-\inf _{\vec{x} \in D(H, \vec{v}, \Gamma) \cap \mathbb{K}^{H}} \sum_{j=1}^{H} v_{j} \mathbb{I}_{i_{j}, \epsilon_{1}, \epsilon_{2}}\left(x_{j}\right)+\alpha \\
\leq-\min _{H} \min _{\underline{i}_{H}} \min _{\vec{v} \in \Omega(H)}-1_{[H \geq 2]} \sum_{j=0}^{H-1}\left(\sum_{l=1}^{j} v_{l}\right) \mathcal{U}_{0}\left(i_{j}, i_{j+1}\right) \\
+\inf _{\vec{x} \in D(H, \vec{v}, \bar{B}) \cap \mathbb{K}^{H}} \sum_{j=1}^{H} v_{j} \mathbb{I}_{i_{j}, \epsilon_{1}, \epsilon_{2}}\left(x_{j}\right)+\alpha \\
\leq-\mathbb{J}_{\mathcal{U}_{0}, \epsilon_{1}, \epsilon_{2}}(\bar{B})+\alpha=-\mathbb{J}_{\mathcal{U}_{0}, \epsilon_{1}, \epsilon_{2}}(\bar{\Gamma} \cap \mathbb{K})+\alpha .
\end{gathered}
$$

As $\alpha$ is arbitrary, the proposition follows.

\section{Lower Coarse Graining Bounds}

As with the lower surgery estimate, the plan will be to restrict the process to conveniently chosen events to derive lower bounds. Recall the notation $A_{n}^{\prime}(l), \underline{i}_{H}, C\left(\underline{i}_{H}\right)$, $\vec{k}_{H}$, and $S_{n}\left(\vec{k}_{H}\right)$ from sections 5 and 8 .

Also, for $l \in \mathcal{G}$, let $\mathbb{P}_{\eta}^{l}$ denote the homogeneous non-negative measure on $C_{l}$ with transition matrix $P(l)$ and initial distribution $\eta$.

Proof of Proposition 4.9. As $\pi$ is (SIE-1)-positive, let $e_{l} \in C_{l}$ be such that $\pi\left(e_{l}\right)>0$ for $l \in \mathcal{G}$. Now, when $M=1, \mathcal{G}=\left\{\zeta_{1}\right\}, \mathbb{J}_{\mathcal{T}_{1}}=\mathbb{I}_{\zeta_{1}}$, and on the set $G_{n}$, the process never leaves $C_{\zeta_{1}}$. In this case,

$$
\underline{\mu}_{\pi}\left(Z_{n} \in \Gamma_{3}, G_{n}\right) \geq \mathbb{P}_{e_{\zeta_{1}}^{\zeta_{1}}}^{\zeta_{1}}\left(Z_{n} \in \Gamma_{3}\right)
$$

and the desired lower bound follows from Proposition 2.1.

Suppose that $M \geq 2$.

Step 1. Let now $\underline{i}_{M}=\left\langle i_{1}, \ldots, i_{M}\right\rangle$ where $i_{j} \in \mathcal{G}$ for $1 \leq j \leq M$ be a given ordering of the nondegenerate irreducible sets $\mathcal{G}$. Let also $\Omega_{M}^{+}=\left\{\vec{v} \in \Omega_{M}: v_{i}>0\right.$ for $1 \leq$ $i \leq M\}$ be the set of positive measures, and let $\vec{v} \in \Omega_{M}^{+}$. Define also $v(0)=0$ 
and $v(u)=\sum_{j=1}^{u} v_{j}$ for $1 \leq u \leq M$, and in addition, for $n$ large enough so that $\lfloor n v(u)\rfloor<\lfloor n v(u+1)\rfloor$ for $1 \leq u \leq M-1$, that $\vec{k}^{n}=\langle\lfloor n v(1)\rfloor, \ldots,\lfloor n v(M-1)\rfloor\rangle$.

Then, for all large $n$,

$$
\begin{aligned}
\underline{\mu}_{\pi}\left(Z_{n} \in \Gamma_{3}, G_{n}\right) & \geq \underline{\mu}_{\pi}\left(Z_{n} \in \Gamma_{3}, A_{n}^{\prime}(M-1), C\left(\underline{i}_{M}\right)\right) \\
& =\sum_{\vec{k}_{M}} \underline{\mu}_{\pi}\left(Z_{n} \in \Gamma_{3}, A_{n}^{\prime}(M-1), C\left(\underline{i}_{M}\right), S_{n}\left(\vec{k}_{M}\right)\right) \\
& \geq \underline{\mu}_{\pi}\left(Z_{n} \in \Gamma_{3}, A_{n}^{\prime}(M-1), C\left(\underline{i}_{M}\right), S_{n}\left(\vec{k}^{n}\right)\right) .
\end{aligned}
$$

Step 2. As $\Gamma_{3}$ is open, the set $D\left(M, \vec{v}, \Gamma_{3}\right)$ (cf. (2.6)) is also open. Then, for $\vec{x} \in D\left(M, \vec{v}, \Gamma_{3}\right)$, let $\epsilon>0$ be so small so that the open cube $\Delta^{\epsilon}(\vec{x})$ about $\vec{x}$ with side length $\epsilon$ is contained $\Delta^{\epsilon}(\vec{x})=\prod_{j=1}^{M} \Delta^{\epsilon}\left(x_{j}\right) \subset D\left(M, \vec{v}_{M}, \Gamma_{3}\right)$. Also, for simplicity, let

$$
E_{n}=A_{n}^{\prime}(M-1) \cap C\left(\underline{i}_{M}\right) \cap S_{n}\left(\vec{k}^{n}\right) \text { and } a_{n}(u, \vec{v})=\frac{1}{v_{u}} \frac{\lfloor n v(u)\rfloor-\lfloor n v(u-1)\rfloor}{n}
$$

for $1 \leq u \leq M$. Then, (9.1) equals

$$
\begin{aligned}
& \underline{\mu}_{\pi}\left(\left\langle a_{n}(1, \vec{v}) Z_{1}^{\lfloor n v(1)\rfloor}, \ldots, a_{n}(M, \vec{v}) Z_{\lfloor n v(M-1)\rfloor+1}^{n}\right\rangle \in D\left(M, \vec{v}, \Gamma_{3}\right), E_{n}\right) \\
& \quad \geq \underline{\mu}_{\pi}\left(\left\langle a_{n}(1, \vec{v}) Z_{1}^{\lfloor n v(1)\rfloor}, \ldots, a_{n}(M, \vec{v}) Z_{\lfloor n v(M-1)\rfloor+1}^{n}\right\rangle \in \Delta^{\epsilon}(\vec{x}), E_{n}\right) .
\end{aligned}
$$

Step 3. To make notation easier, we now get "rid" of the $a_{n}(u, \vec{v})$ terms at the cost of a further lower bound. Namely, as $Z_{i}^{u} \in \mathbb{K}$ is bounded for all $1 \leq i \leq u$, and $a_{n}(u, \vec{v}) \rightarrow 1$ for $1 \leq u \leq M$, we have for all $n$ large enough that

$$
\begin{aligned}
& \left\{\left\langle Z_{1}^{\lfloor n v(1)\rfloor}, Z_{\lfloor n v(1)\rfloor+2}^{\lfloor n v(2)\rfloor}, \ldots, Z_{\lfloor n v(M-1)\rfloor+2}^{n}\right\rangle \in \Delta^{\epsilon / 2}(\vec{x})\right\} \\
& \subset\left\{\left\langle a_{n}(1, \vec{v}) Z_{1}^{\lfloor n v(1)\rfloor}, \ldots, a_{n}(M, \vec{v}) Z_{\lfloor n v(M-1)\rfloor+1}^{n}\right\rangle \in \Delta^{\epsilon}(\vec{x})\right\} .
\end{aligned}
$$

Therefore, dropping the superscript $\Delta(\vec{x})=\Delta^{\frac{\epsilon}{2}}(\vec{x})$, we have for large $n$ that

$$
(9.2) \geq \underline{\mu}_{\pi}\left(\left\langle Z_{1}^{\lfloor n v(1)\rfloor}, Z_{\lfloor n v(1)\rfloor+2}^{\lfloor n v(2)\rfloor}, \ldots, Z_{\lfloor n v(M-1)\rfloor+2}^{n}\right\rangle \in \Delta(\vec{x}), E_{n}\right) .
$$

Step 4. We now decompose (9.3) in terms of resting and routing transitions. Recall that the transition probability between states $x \in C_{l}$ and $y \in C_{m}$ at time $n$ with respect to $\underline{\mu}_{\pi}$ equals $\check{\gamma}^{0}(n+1,(l, m))$ and does not depend on atoms $x$ and $y$. 
Bound (9.3) below by

$$
\begin{aligned}
& \pi\left(e_{i_{1}}\right) \underline{\mu}_{e_{i_{1}}}\left(\left\langle Z_{1}^{\lfloor n v(1)\rfloor}, Z_{\lfloor n v(1)\rfloor+2}^{\lfloor n v(2)\rfloor}, \ldots, Z_{\lfloor n v(M-1)\rfloor+2}^{n}\right\rangle \in \Delta(\vec{x}),\right. \\
& \left.X_{\lfloor n v(u-1)\rfloor}=e_{i_{u}} \text { and } X_{\lfloor n v(u-1)\rfloor+1}=e_{i_{u+1}} \text { for } 2 \leq u \leq M-1, E_{n}\right) \\
& =\prod_{u=1}^{M-1} \check{\gamma}^{0}\left(\lfloor n v(u)\rfloor+1,\left(i_{u}, i_{u+1}\right)\right) \cdot \mathbb{P}_{e_{i_{1}}}^{i_{1}}\left(Z_{1}^{\lfloor n v(1)\rfloor} \in \Delta\left(x_{1}\right), X_{\lfloor n v(1)\rfloor}=e_{i_{2}}\right) \\
& \cdot \prod_{u=2}^{M-1} \mathbb{P}_{\left(\lfloor n v(u-1)\rfloor+1, e_{\left.i_{u}\right)}\right.}^{i_{u}}\left(Z_{\lfloor n v(u-1)\rfloor+2}^{\lfloor n v(u)\rfloor} \in \Delta\left(x_{u}\right), X_{\lfloor n v(u)\rfloor}=e_{i_{u+1}}\right) \\
& \cdot \mathbb{P}_{\left(\lfloor n v(M-1)\rfloor+1, e_{i_{M}}\right)}^{i_{M}}\left(Z_{\lfloor n v(M-1)\rfloor+2}^{n} \in \Delta\left(x_{M}\right)\right) .
\end{aligned}
$$

Step 5. Observe, by definition, for distinct $i, j \in \mathcal{G}$, that

$$
\underline{\lim } \frac{1}{k} \log \check{\gamma}^{0}(k,(i, j))=\underline{\lim } \frac{1}{k} \log \min _{0 \leq r \leq E(N, M)} \underline{\gamma}^{1}(k+r,(i, j))=\mathcal{T}_{1}(i, j) .
$$

Then, as large deviations of finite time-homogeneous irreducible chains are independent of the first and last observations, we have

$$
\begin{aligned}
& \underline{\lim } \frac{1}{n} \log (9.4) \\
& \geq \sum_{u=1}^{M-1}\left(\underline{\lim } \frac{\lfloor n v(u)\rfloor+1}{n}\right)\left(\underline{\lim } \frac{1}{\lfloor n v(u)\rfloor+1} \log \check{\gamma}^{0}\left(\lfloor n v(u)\rfloor+1,\left(i_{u}, i_{u+1}\right)\right)\right) \\
& -\sum_{u=1}^{M} v_{u} \mathbb{I}_{i_{u}}\left(\Delta\left(x_{u}\right)\right) \\
& \geq \sum_{u=1}^{M-1} v(u) \mathcal{T}_{1}\left(\zeta_{i_{u}}, \zeta_{i_{u+1}}\right)-\sum_{u=1}^{M} v_{u} \mathbb{I}_{i_{u}}\left(x_{u}\right)
\end{aligned}
$$

Step 6. As $\vec{v} \in \Omega_{M}^{+}, \vec{x} \in D(M, \vec{v}, \Gamma)$, and arrangement $\underline{i}_{M}$ composed of members in $\mathcal{G}$ are arbitrary, we have from (9.5) that

$$
\underline{\lim } \frac{1}{n} \log \underline{\mu}_{\pi}\left(Z_{n} \in \Gamma_{3}, G_{n}\right) \geq \sup _{\vec{v} \in \Omega_{M}^{+}} \max _{\sigma \in \mathbb{S}_{M}} g(\vec{v}, \sigma)
$$

where

$$
g(\vec{v}, \sigma)=\sum_{u=1}^{M-1} v(u) \mathcal{T}_{1}\left(\zeta_{\sigma(u)}, \zeta_{\sigma(u+1)}\right)-\inf _{\vec{y} \in D\left(M, \vec{v}, \Gamma_{3}\right)} \sum_{u=1}^{M} v_{u} \mathbb{I}_{\zeta_{\sigma(u)}}\left(y_{u}\right) .
$$

We now argue that we can replace $\Omega_{M}^{+}$with the larger $\Omega_{M}$ in (9.6). In Lemma 9.1 below we show, for each $\sigma$, that $g(\cdot, \sigma)$ is lower semicontinuous as a function on $\Omega_{M}$. 
In particular, as $\mathbb{S}_{M}$ is a finite set, $\max _{\sigma \in \mathbb{S}_{M}} g(\cdot, \sigma)$ is lower semi-continous. Therefore, by taking limits, we improve the bound in (9.6) to

$$
\underline{\lim } \frac{1}{n} \log \underline{\mu}_{\pi}\left(Z_{n} \in \Gamma_{3}, G_{n}\right) \geq \sup _{\vec{v} \in \Omega_{M}} \max _{\sigma \in \mathbb{S}_{M}} g(\vec{v}, \sigma)
$$

which is identified as $-\inf _{z \in \Gamma} \mathbb{J}_{\mathcal{T}_{1}}(z)$.

Lemma 9.1 Let $B \subset \mathbb{R}^{d}$ be an open set, and let $M \geq 2$ and $\sigma \in \mathbb{S}_{M}$. Then, $g(\cdot, \sigma)$ : $\Omega_{M} \rightarrow[0, \infty]$ is lower semicontiuous.

Proof. Let $\left\{\vec{v}^{n}\right\} \subset \Omega_{M}$ be a sequence which converges, $\vec{v}^{n} \rightarrow \vec{v}$. Recalling our convention, $0 \cdot(-\infty)=0$, we note that $h_{1}(\vec{v})=\sum_{u=1}^{M-1} v(u) \mathcal{T}_{1}\left(\zeta_{\sigma(u)}, \zeta_{\sigma(u+1)}\right)$ is lower semicontinous, and so we need only prove $h_{2}(\vec{v})=\inf _{\vec{y} \in D(M, \vec{v}, B)} \sum_{u=1}^{M} v_{u} \mathbb{I}_{\zeta_{\sigma(u)}}\left(y_{u}\right)$ is upper semicontinuous.

Let now $\vec{w} \in D(M, \vec{v}, B)$. Because $B$ is open and $\vec{v}^{n}$ converges to $\vec{v}$, we must have $\vec{w} \in D\left(M, \vec{v}^{n}, B\right)$ for all large $n$. Then,

$$
\varlimsup h_{2}\left(\vec{v}^{n}\right) \leq \varlimsup \sum_{u=1}^{M} v_{u}^{n} \mathbb{I}_{\zeta_{\sigma(u)}}\left(w_{u}\right)=\sum_{u=1}^{M} v_{u} \mathbb{I}_{\zeta_{\sigma(u)}}\left(w_{u}\right) .
$$

However, as $\vec{w} \in D(M, \vec{v}, B)$ is arbitrary, we have in fact that

$$
\varlimsup h_{2}\left(\vec{v}^{n}\right) \leq \inf _{\vec{y} \in D(M, \vec{v}, B)} \sum_{u=1}^{M} v_{u} \mathbb{I}_{\zeta_{\sigma(u)}}\left(y_{u}\right)=h_{2}(\vec{v}) .
$$

\section{Limit Estimate on $\mathbb{J}_{\mathcal{U}_{0}, \epsilon_{1}, \epsilon_{2}}$}

The proof of Proposition 4.5 follows in two steps (Propositions 10.1 and 10.2). The first step is to take $\epsilon_{1} \downarrow 0$ and estimate in terms of a quantity independent of degenerate transient sets $\mathcal{D}$ in Proposition 10.1. The second step is to let $\epsilon_{2} \downarrow 0$ and recover $\mathbb{J}_{\mathcal{U}_{0}}$ in the limit in Proposition 10.2.

It will be helpful to reduce the expression $\mathbb{J}_{\mathcal{U}_{0}, \epsilon_{1}, \epsilon_{2}}$ for $\epsilon_{1}, \epsilon_{2}>0$ (cf. (4.2)). As $\mathbb{I}_{i, \epsilon_{1}, \epsilon_{2}}$ is degenerate around $f(i)$ for $i \in \mathcal{D}$ (cf. (4.1)), we can evaluate $\mathbb{U}_{\mathcal{U}_{0}, \epsilon_{1}, \epsilon_{2}}(B)$ for $B \subset \mathbb{R}^{d}$ and $N+M \geq 2$ as

$$
\begin{aligned}
& \min _{\sigma \in \mathbb{S}_{N+M}} \inf _{\vec{v} \in \Omega_{N+M}} \inf _{\vec{x} \in D^{\prime}(\vec{v})} \\
& \left\{-\sum_{i=1}^{N+M-1} \mathcal{U}_{0}(\sigma(i), \sigma(i+1))\left[\sum_{j=1}^{i} v_{j}\right]-\sum_{\sigma(i) \in \mathcal{D}} v_{i} \log \epsilon_{1}+\sum_{\sigma(i) \in \mathcal{G}} v_{i} I_{\sigma(i), \epsilon_{2}}\left(x_{i}\right)\right\}
\end{aligned}
$$


where $D^{\prime}(\vec{v})=\left\{\vec{x} \in D(N+M, \vec{v}, B): x_{i}=f(\sigma(i))\right.$, for $\left.\sigma(i) \in \mathcal{D}\right\}$.

When $N+M=1$, the formula collapses to $\mathbb{J}_{\mathcal{U}_{0}, \epsilon_{1}, \epsilon_{2}}=\mathbb{I}_{1, \epsilon_{2}}$.

We describe now an $\epsilon_{2} \geq 0$ "perturbation" of $\mathbb{J}_{\mathcal{U}_{0}}$ where one replaces rates $\mathbb{I}_{i}$ with $\mathbb{I}_{i, \epsilon_{2}}$ for $i \in \mathcal{G}$. Define, for Borel $B \subset \mathbb{R}^{d}$ and $M \geq 2$, that

$$
\mathbb{J}_{\mathcal{U}_{0}}^{\epsilon_{2}}(B)=\min _{\sigma \in \mathbb{S}_{M}} \inf _{\vec{v} \in \Omega_{M}} \inf _{\vec{x} \in D(M, \vec{v}, B)}-\sum_{i=1}^{M-1} \mathcal{U}_{0}\left(\zeta_{\sigma(i)}, \zeta_{\sigma(i+1)}\right)\left[\sum_{j=1}^{i} v_{j}\right]+\sum_{i=1}^{M} v_{i} I_{\zeta_{\sigma(i)}, \epsilon_{2}}\left(x_{i}\right) .
$$

When $M=1$, let $\mathbb{J}_{\mathcal{U}_{0}}^{\epsilon_{2}}=\mathbb{I}_{1, \epsilon_{2}}$.

We give now a triangle cost bound useful for the first step.

Lemma 10.1 For distinct $i, j, k \in \mathcal{G}$,

$$
\mathcal{U}_{0}(i, j)+\mathcal{U}_{0}(j, k) \leq \mathcal{U}_{0}(i, k) .
$$

Proof. By definition, for some $k_{1}$ and distinct elements $L^{1}=\left\langle l_{0}^{1}=i, l_{1}^{1}, \ldots, l_{k_{1}}^{1}, l_{k_{1}+1}^{1}=\right.$ $j\rangle$ we have $\mathcal{U}_{0}(i, j)=\sum_{s=0}^{k_{1}} v\left(l_{s}^{1}, l_{s+1}^{1}\right)$. Similarly, we have for some $k_{2}$ and $L^{2}=\left\langle l_{0}^{2}=\right.$ $\left.j, l_{1}^{2}, \ldots, l_{k_{2}}^{2}, l_{k_{2}+1}^{2}=k\right\rangle$ that $\mathcal{U}_{0}(j, k)=\sum_{s=0}^{k_{2}} v\left(l_{s}^{2}, l_{s+1}^{2}\right)$. Let now $T$ be the first index of an element in $L^{1}$ which belongs to $L^{2}$. Clearly, $1<T \leq k_{1}+1$. Call also $T^{\prime}$ the index of this element in $L^{2}$.

Form now $L^{3}=\left\langle l_{0}^{1}, l_{1}^{1}, \ldots, l_{T}^{1}, l_{T^{\prime}+1}^{2}, \ldots, l_{k_{2}+1}^{2}\right\rangle$. From construction, $L^{3}$ is a list of distinct elements which we will relabel as $L^{3}=\left\langle l_{0}^{3}, \ldots, l_{k_{3}}^{3}\right\rangle$ for some $k_{3}$.

Now, since $v(a, b) \leq 0$ for all distinct $a, b$, we have

$$
\sum_{s=0}^{k_{3}} v\left(l_{s}^{3}, l_{s+1}^{3}\right) \geq \sum_{s=0}^{k_{1}} v\left(l_{s}^{1}, l_{s+1}^{1}\right)+\sum_{s=0}^{k_{2}} v\left(l_{s}^{2}, l_{s+1}^{2}\right) .
$$

However,

$$
\mathcal{U}_{0}(i, k)=\max _{0 \leq k \leq M-2} \max _{L_{k}} \sum_{s=0}^{k} v\left(l_{s}, l_{s+1}\right) \geq \sum_{s=0}^{k_{3}} v\left(l_{s}^{3}, l_{s+1}^{3}\right) \geq \mathcal{U}_{0}(i, j)+\mathcal{U}_{0}(j, k) .
$$

Proposition 10.1 Let $B \subset \mathbb{K}$ be a compact set, and fix $\epsilon_{2} \geq 0$. Then, we have

$$
\underline{\lim _{\epsilon_{1} \downarrow 0}} \mathbb{J}_{\mathcal{U}_{0}, \epsilon_{1}, \epsilon_{2}}(B) \geq \mathbb{J}_{\mathcal{U}_{0}}^{\epsilon_{2}}(B) .
$$

Proof. First, when $N=0$, we inspect that $\mathbb{J}_{\mathcal{U}_{0}, \epsilon_{1}, \epsilon_{2}}(B)=\mathbb{J}_{\mathcal{U}_{0}}^{\epsilon_{2}}(B)$. Therefore, we assume that $N \geq 1$ in the following. 
Step 1. Let $\epsilon(k) \downarrow 0, \vec{v}^{\epsilon(k)}, \vec{x}^{\epsilon(k)}$ and $\sigma_{\epsilon(k)}$ be sequences so that the limit inferior is attained,

$$
\begin{aligned}
\underline{\varliminf_{\epsilon} \downarrow 0} \mathbb{J}_{\mathcal{U}_{0}, \epsilon_{1}, \epsilon_{2}}(B) \\
=\lim _{k \rightarrow \infty}-\sum_{i=1}^{N+M-1} \mathcal{U}_{0}\left(\sigma_{\epsilon(k)}(i), \sigma_{\epsilon(k)}(i+1)\right)\left[\sum_{j=1}^{i} v_{j}^{\epsilon(k)}\right] \\
\quad-\sum_{\sigma_{\epsilon(k)}(i) \in \mathcal{D}} v_{i}^{\epsilon(k)} \log \epsilon(k)+\sum_{\sigma_{\epsilon(k)}(i) \in \mathcal{G}} v_{i}^{\epsilon(k)} \mathbb{I}_{\sigma_{\epsilon(k)}(i), \epsilon(k)}\left(x_{i}^{\epsilon(k)}\right) .
\end{aligned}
$$

As $\Omega_{N+M}$ is compact and $\mathbb{S}_{N+M}$ is finite, a further subsequence may be found so that, with the same labels, $\vec{v}^{\epsilon(k)} \rightarrow \vec{v}$, and $\sigma_{\epsilon(k)}=\sigma$ for all small $\epsilon_{1}$.

Step 2. When $\sum_{\sigma(i) \in \mathcal{D}} v_{i}>0$, we have (10.1) diverges to $\infty$ which is automatically greater than the right-side in the proposition.

On the other hand, if $\sum_{\sigma(i) \in \mathcal{D}} v_{i}=0$, we must have $\sum_{\sigma(i) \in \mathcal{G}} v_{i}=1$ as $\vec{v}$ is a probability vector. Now, if $(10.1)=\infty$, the proposition bound again holds.

Suppose therefore that (10.1) is finite. Recall that the cube $\mathbb{K}$ contains the the domains of finiteness of the rate functions $\left\{\mathbb{I}_{i, \epsilon_{2}}: i \in \mathcal{G}\right\}$ (cf. Proposition 2.1). Therefore, by taking a subsequence and relabeling, we can take $\vec{x}^{\epsilon(k)} \in D^{\prime}\left(\vec{v}^{\epsilon(k)}\right) \cap \mathbb{K}$ and ensure the sequence is convergent, $\vec{x}^{\epsilon(k)} \rightarrow \vec{x}$. Moreover, $\vec{x} \in D(N+M, \vec{v}, B)$ since $\sum_{i=1}^{N+M} v_{i}^{\epsilon(k)} x_{i}^{\epsilon(k)} \in B \rightarrow \sum_{i=1}^{N+M} v_{i} x_{i}$ and $B$ is closed.

Then, as $-\sum_{\sigma(i) \in \mathcal{D}} v_{i}^{\epsilon(k)} \log \epsilon(k) \geq 0$ and the rate functions $\mathbb{I}_{i, \epsilon_{2}}$ are lower semicontinous, we have that

$$
\begin{aligned}
(10.1) & \geq \underline{\lim }_{k \rightarrow \infty}-\sum_{i=1}^{N+M-1} \mathcal{U}_{0}(\sigma(i), \sigma(i+1))\left[\sum_{j=1}^{i} v_{j}^{\epsilon(k)}\right]+\sum_{\sigma(i) \in \mathcal{G}} v_{i}^{\epsilon(k)} \mathbb{I}_{\sigma(i), \epsilon_{2}}\left(x_{i}^{\epsilon(k)}\right) \\
& \geq-\sum_{i=1}^{N+M-1} \mathcal{U}_{0}(\sigma(i), \sigma(i+1))\left[\sum_{j=1}^{i} v_{j}\right]+\sum_{\sigma(i) \in \mathcal{G}} v_{i} \mathbb{I}_{\sigma(i), \epsilon_{2}}\left(x_{i}\right)
\end{aligned}
$$

Step 3. When $M=1$ and $N \geq 1$, then $\mathcal{G}=\left\{\zeta_{1}\right\}$ is a singleton, and $v_{\zeta_{1}}=1$. Moreover, as $-\mathcal{U}_{0}$ is non-negative, $(10.2)$ is bounded below by $\mathbb{I}_{\zeta_{1}, \epsilon_{2}}\left(x_{\zeta_{1}}\right)=\mathbb{J}_{\mathcal{U}_{0}}^{\epsilon_{2}}$ to finish the proof in this case.

Step 4. Suppose then that $M \geq 2$ and $N \geq 1$. The strategy will be to form a permutation $\eta \in \mathbb{S}_{M}$ and vector $\vec{u} \in \Omega_{M}$ for which (10.2) reduces to an expression involving only terms relating to $\mathcal{G}$. Write $\sigma^{-1}(\mathcal{G})=\left\{\chi_{1}, \ldots, \chi_{M}\right\}$ where $\chi_{i}$ is ordered as follows,

$$
\chi_{1}=\min \{s: \sigma(s) \in \mathcal{G}\} \text { and } \chi_{i}=\min \left\{s>X_{i-1}: \sigma(s) \in \mathcal{G}\right\} \quad \text { when } 2 \leq i \leq M .
$$


Now, as $v_{i}=0$ for $\sigma(i) \notin \mathcal{G}$, and in particular $v_{i}=0$ for $1 \leq i \leq \chi_{1}-1$ when $\chi_{1} \geq 2$, we have

$$
\begin{aligned}
-\sum_{i=1}^{N+M-1} \mathcal{U}_{0}(\sigma(i), \sigma(i+1))\left[\sum_{j=1}^{i} v_{j}\right] \\
=-\sum_{i=\chi_{1}}^{N+M-1} \mathcal{U}_{0}(\sigma(i), \sigma(i+1)) \sum_{\substack{\chi_{1} \leq j \leq i \\
j \in \sigma^{-1}(\mathcal{G})}} v_{j} \\
=-\sum_{k=1}^{M-1} \sum_{i=\chi_{k}}^{\chi_{k+1}-1} \mathcal{U}_{0}(\sigma(i), \sigma(i+1)) \sum_{\substack{\chi_{1} \leq j \leq i \\
j \in \sigma^{-1}(\mathcal{G})}} v_{j}+K_{0}
\end{aligned}
$$

where

$$
K_{0}= \begin{cases}-\sum_{i=\chi_{M}}^{N+M-1} \mathcal{U}_{0}(\sigma(i), \sigma(i+1))\left[\sum_{\substack{1 \leq j \leq i \\ j \in \sigma^{-1}(\mathcal{G})}} v_{j} \quad \text { when } \chi_{M}<N+M\right. \\ 0 & \text { when } \chi_{M}=N+M .\end{cases}
$$

In any case, as $K_{0}$ is non-negative, we have that

$$
-\sum_{i=1}^{N+M-1} \mathcal{U}_{0}(\sigma(i), \sigma(i+1))\left[\sum_{j=1}^{i} v_{j}\right] \geq-\sum_{k=1}^{M-1}\left[\sum_{i=\chi_{k}}^{\chi_{k+1}-1} \mathcal{U}_{0}(\sigma(i), \sigma(i+1)) \sum_{\substack{\chi_{1} \leq j \leq i \\ j \in \sigma^{-1}(\mathcal{G})}} v_{j}\right] \text {. }
$$

Step 5. We now bound individually the terms in large brackets in (10.3). For each $\chi_{k} \leq i \leq \chi_{k+1}-1$, as $\left\{v_{j}: \chi_{1} \leq j \leq i\right.$, and $\left.j \in \sigma^{-1}(\mathcal{G})\right\}=\left\{v_{\chi_{s}}: 1 \leq s \leq k\right\}$, we may write

$$
\begin{gathered}
\sum_{i=\chi_{k}}^{\chi_{k+1}-1} \mathcal{U}_{0}(\sigma(i), \sigma(i+1)) \sum_{\substack{\chi_{1} \leq j \leq i \\
j \in \sigma^{-1}(\mathcal{G})}} v_{j} \\
=\left[\mathcal{U}_{0}\left(\sigma\left(\chi_{k}\right), \sigma\left(\chi_{k}+1\right)\right)+\mathcal{U}_{0}\left(\sigma\left(\chi_{k}+1\right), \sigma\left(\chi_{k}+2\right)\right)\right. \\
\left.+\cdots+\mathcal{U}_{0}\left(\sigma\left(\chi_{k+1}-1\right), \sigma\left(\chi_{k+1}\right)\right)\right]\left[\sum_{s=1}^{k} v_{\chi_{s}}\right] \\
\leq \mathcal{U}_{0}\left(\sigma\left(\chi_{k}\right), \sigma\left(\chi_{k+1}\right)\right) \sum_{s=1}^{k} v_{\chi_{s}}
\end{gathered}
$$

by repeatedly applying the "triangle inequality" Lemma 10.1.

Hence, pulling together the inequalities, we have

$$
-\sum_{i=1}^{N+M-1} \mathcal{U}_{0}(\sigma(i), \sigma(i+1))\left[\sum_{j=1}^{i} v_{j}\right] \geq-\sum_{k=1}^{M-1} \mathcal{U}_{0}\left(\sigma\left(\chi_{k}\right), \sigma\left(\chi_{k+1}\right)\right)\left[\sum_{s=1}^{k} v_{\chi_{s}}\right] .
$$


Step 6 . Define now $\vec{u} \in \Omega_{M}$ by $u_{k}=v_{\chi_{k}}$ for $1 \leq k \leq M$. Then,

$$
\sum_{i \in \sigma^{-1}(\mathcal{G})} v_{i} \mathbb{I}_{\sigma(i), \epsilon_{2}}\left(x_{i}\right)=\sum_{k=1}^{M} v_{\chi_{k}} \mathbb{I}_{\sigma\left(\chi_{k}\right), \epsilon_{2}}\left(x_{\chi_{k}}\right)=\sum_{k=1}^{M} u_{k} \mathbb{I}_{\sigma\left(\chi_{k}\right), \epsilon_{2}}\left(x_{\chi_{k}}\right) .
$$

Let now $\eta \in \mathbb{S}_{M}$ be the permutation where $\zeta_{\eta(i)}=\sigma\left(\chi_{i}\right)$ for $1 \leq i \leq M$. Noting (10.4), we can then bound (10.2) below by

$$
\begin{array}{r}
-\sum_{k=1}^{M-1} \mathcal{U}_{0}\left(\sigma\left(\chi_{k}\right), \sigma\left(\chi_{k+1}\right)\right)\left[\sum_{s=1}^{k} u_{s}\right]+\sum_{k=1}^{M} u_{k} \mathbb{I}_{\sigma\left(\chi_{k}\right), \epsilon_{2}}\left(x_{\chi_{k}}\right) \\
=-\sum_{k=1}^{M-1} \mathcal{U}_{0}\left(\zeta_{\eta(k)}, \zeta_{\eta(k+1)}\right)\left[\sum_{s=1}^{k} u_{s}\right]-\sum_{k=1}^{M} u_{k} \mathbb{I}_{\zeta_{\eta(k)}, \epsilon_{2}}\left(x_{\chi_{k}}\right) .
\end{array}
$$

Step 7. By construction $\sum_{i=1}^{N+M} v_{i} x_{i} \in B$. Then, as $v_{j}=0$ when $\sigma(j) \notin \mathcal{G}$, we have

$$
\sum_{j=1}^{N+M} v_{j} x_{j}=\sum_{j \in \sigma^{-1}(\mathcal{G})} v_{j} x_{j}=\sum_{j \in \sigma^{-1}(\mathcal{G})} v_{j} x_{j}=\sum_{s=1}^{M} v_{\chi_{s}} x_{\chi_{s}}=\sum_{s=1}^{M} u_{s} x_{\chi_{s}}
$$

and so $\left\langle x_{\chi_{1}}, \ldots, x_{\chi_{M}}\right\rangle \in D(M, \vec{u}, B)$. Hence, tracing through the argument,

$$
\begin{aligned}
(10.5) & \geq \inf _{\vec{x} \in D(M, \vec{u}, B)}-\sum_{k=1}^{M-1} \mathcal{U}_{0}\left(\zeta_{\eta(k)}, \zeta_{\eta(k+1)}\right)\left[\sum_{s=1}^{k} u_{s}\right]-\sum_{k=1}^{M} u_{k} I_{\zeta_{\eta(k)}, \epsilon_{2}}\left(x_{k}\right) \\
& \geq \mathbb{J}_{\mathcal{U}_{0}}^{\epsilon_{2}}(B) .
\end{aligned}
$$

Proposition 10.2 Let $\Gamma \subset \mathbb{R}^{d}$ be compact. Then, we have

$$
\varliminf_{\epsilon \downarrow} \mathbb{U}_{\mathcal{U}_{0}}^{\epsilon}(\Gamma) \geq \mathbb{J}_{\mathcal{U}_{0}}(\Gamma) .
$$

Proof. When $\underline{\lim }_{\epsilon \downarrow} \mathbb{J}_{\mathcal{U}_{0}}^{\epsilon}(\Gamma)=\infty$, of course (10.6) is immediate.

Step 1. Suppose then that $\underline{\lim }_{\epsilon \downarrow 0} \mathbb{J}_{\mathcal{U}_{0}}^{\epsilon}(\Gamma)<\infty$. As in Step 2 in Proposition 10.1 above, let $\epsilon(k) \downarrow 0, \sigma_{\epsilon(k)}=\sigma$ independent of $k, \vec{v}^{\epsilon(k)} \rightarrow \vec{v}$, and $\vec{x}^{\epsilon(k)} \rightarrow \vec{x} \in D(M, \vec{v}, \Gamma)$ be such that

$$
\underline{\lim }_{\epsilon \downarrow 0} \mathbb{J}_{\mathcal{U}_{0}}^{\epsilon}(\Gamma)=\lim _{k \rightarrow \infty}-\sum_{i=1}^{M-1}\left(\sum_{j=1}^{i} v_{j}^{\epsilon(k)}\right) \mathcal{U}_{0}\left(\left(\zeta_{\sigma(i)}, \zeta_{\sigma(i+1)}\right)+\sum_{i=1}^{M} v_{i}^{\epsilon(k)} \mathbb{I}_{\zeta_{\sigma(i)}, \epsilon(k)}\left(x_{i}^{\epsilon(k)}\right) .\right.
$$

Step 2. We now claim for $i \in \mathcal{G}$ that

$$
\varliminf_{k \rightarrow \infty} \mathbb{I}_{i, \epsilon(k)}\left(x_{i}^{\epsilon(k)}\right) \geq \mathbb{I}_{i}\left(x_{i}\right) .
$$


For $\lambda \in \mathbb{R}^{d}$, let $\rho_{i, \epsilon}(\lambda)$ and $\rho_{i}(\lambda)$ be the Perron-Frobenius eigenvalues corresponding to the $\lambda$-tilts of $P(i, \epsilon)$ and $P(i)$ (cf. (2.3)). From Lancaster [21], we have that $\lim _{\epsilon \downarrow 0} \log \rho_{i, \epsilon}(\lambda)=\log \rho_{i}(\lambda)$.

Now, for $\lambda^{\prime} \in \mathbb{R}^{d}$, observe that

$$
\begin{aligned}
\frac{\lim }{k} \mathbb{I}_{i, \epsilon(k)}\left(x_{i}^{\epsilon(k)}\right) & =\frac{\lim }{k} \sup _{\lambda \in \mathbb{R}^{d}}\left\langle\lambda, x_{i}^{\epsilon(k)}\right\rangle-\log \rho_{i, \epsilon(k)}(\lambda) \\
& \geq \frac{\lim }{k}\left\langle\lambda^{\prime}, x_{i}^{\epsilon(k)}\right\rangle-\log \rho_{i, \epsilon(k)}\left(\lambda^{\prime}\right)=\left\langle\lambda^{\prime}, x\right\rangle-\log \rho_{i}\left(\lambda^{\prime}\right) .
\end{aligned}
$$

Hence, as $\lambda^{\prime}$ is arbitrary, we have $\underline{\lim }_{k} \mathbb{I}_{i, \epsilon(k)}\left(x_{i}^{\epsilon(k)}\right) \geq \sup _{\lambda}\left\{\langle\lambda, x\rangle-\log \rho_{i}(\lambda)\right\}=\mathbb{I}_{i}(x)$.

Step 3. In fact, (10.7) proves the proposition when $M=1$. On the other hand, when $M \geq 2$, we have with (10.7) that

$$
\varliminf_{\epsilon \downarrow 0} \mathbb{J}_{\mathcal{U}_{0}}^{\epsilon}(\Gamma) \geq-\sum_{i=1}^{M-1}\left(\sum_{j=1}^{i} v_{j}\right) \mathcal{U}_{0}\left(\left(\zeta_{\sigma(i)}, \zeta_{\sigma(i+1)}\right)+\sum_{i=1}^{M} v_{i} \mathbb{I}_{\zeta_{\sigma(i)}}\left(x_{i}\right) \geq \mathbb{J}_{\mathcal{U}_{0}}(\Gamma) .\right.
$$

\section{Routing Cost Comparisions}

We separate the proof of Proposition 4.10 into two separate results.

Proposition 11.1 Suppose Assumption B holds. Then, for distinct $i, j \in \mathcal{G}(P)$,

$$
\mathcal{T}_{1}(i, j) \geq \mathcal{T}_{0}(i, j)
$$

Proof. Recall the definitions of $\underline{\gamma}^{1}(n, y, z)$ and $\underline{\gamma}^{1}(n,(i, j))$. It is enough to prove for $y \in C_{i}$ and $z \in C_{j}$ that

$$
\underline{\lim _{n \rightarrow \infty}} \frac{1}{n} \log \underline{\gamma}^{1}(n, y, z) \geq \mathcal{T}_{0}(i, j)
$$

Then, clearly

$$
\mathcal{T}_{1}(i, j)=\varliminf_{n \rightarrow \infty} \frac{1}{n} \log \underline{\gamma}^{1}(n,(i, j)) \geq \mathcal{T}_{0}(i, j)
$$

finishing the proof.

We now show (11.1). Let $k$ and $L_{k}=\left\langle i=l_{0}, l_{1}, \ldots, l_{k}, l_{k+1}=j\right\rangle$ be such that

$$
\mathcal{T}_{0}(i, j)=\sum_{s=0}^{k} \tau\left(l_{s}, l_{s+1}\right)
$$


To connect with the definition of $\underline{\gamma}^{1}(n,(i, j))$, form vectors $\vec{x}^{0}=\left\langle x_{1}^{0}, \ldots, x_{q_{0}}^{0}\right\rangle, \ldots, \vec{x}^{k+1}=$ $\left\langle x_{1}^{k+1}, \ldots, x_{q_{k+1}}^{k+1}\right\rangle$ with elements in $C_{l_{0}}, \ldots, C_{l_{k+1}}$ such that, for $0 \leq s \leq k$,

$$
x_{q_{s}}^{s}=a\left(l_{s}, l_{s+1}\right), \text { and } x_{1}^{s+1}=b_{n+r(s)}\left(l_{s}, l_{s+1}\right),
$$

$1 \leq q_{s} \leq \mathfrak{r}+1$, and $1 \leq q_{0}, q_{k+1} \leq \mathfrak{r}$. In addition, as $\{P(i): i \in \mathcal{G}\}$ are irreducible, we specify that the paths are possible. Namely, for all large $n$,

$$
\mathbb{P}_{(n-1, y)}\left(\vec{X}_{n}^{n+r(0)-1}=\vec{x}^{0}\right) \geq\left(p_{\min } / 2\right)^{\mathfrak{r}}, \mathbb{P}_{\left(n+r(s-1), x_{1}^{s}\right)}\left(\vec{X}_{n+r(s-1)+1}^{n+r(s)-1}=\vec{x}_{2}^{s}\right) \geq\left(p_{\min } / 2\right)^{\mathfrak{r}},
$$

and

$$
\mathbb{P}_{\left(n+r(k+1)-1, x_{q_{k+1}}^{k+1}\right)}\left(X_{n+r(k+1)}=z\right) \geq p_{\min } / 2
$$

when $q_{s} \geq 2$ and $1 \leq s \leq k+1$. Here, $\vec{x}_{2}^{s}=\left\langle x_{2}^{s}, \ldots, x_{q_{s}}^{s}\right\rangle$ when $q_{s} \geq 2, r(s)=\sum_{u=0}^{s} q_{u}$, and $p_{\min }$ is defined in (2.5).

Since the length of the connecting path from $y$ to $z$ is at most $E_{0}(N, M)$ (cf. near $(2.10))$, we have

$$
\begin{aligned}
& \underline{\lim _{n \rightarrow \infty}} \frac{1}{n} \log \underline{\gamma}^{1}(n, y, z) \\
& \geq \underline{\lim }\left[\frac{\log \left(p_{\min } / 2\right)^{E_{0}(N, M)}}{n}+\frac{1}{n} \sum_{s=0}^{k+1} \log p_{n+r(s)}\left(a\left(l_{s}, l_{s+1}\right), b_{n+r(s)}\left(l_{s}, l_{s+1}\right)\right)\right] \\
& =\sum_{s=0}^{k+1} \tau\left(l_{s}, l_{s+1}\right)=\mathcal{T}_{0}(i, j)
\end{aligned}
$$

from Assumption B.

Proposition 11.2 Suppose that Assumption $C$ holds. Then, for distinct $i, j \in \mathcal{G}$,

$$
\mathcal{T}_{1}(i, j) \geq \mathcal{T}_{0}(i, j)
$$

Proof. The proof is similar to that of Proposition 11.1. As before, it is enough to show (11.1). Let $k$ and $L_{k}=\left\langle i=l_{0}, l_{1}, \ldots, l_{k}, l_{k+1}=j\right\rangle$ be such that $\mathcal{T}_{0}(i, j)=$ $\sum_{s=0}^{k} \tau\left(l_{s}, l_{s+1}\right)$. Form the path vector $\vec{x}^{0}=\left\langle x_{1}^{0}, \ldots, x_{q_{0}}^{0}\right\rangle$ with $1 \leq q_{0} \leq \mathfrak{r}$ in $C_{i}$ and state $x_{1}^{1} \in C_{l_{1}}$ such that

$$
p_{n-1+\left(q_{0}+1\right)}\left(x_{q_{0}}^{0}, x_{1}^{1}\right)=t\left(n+q_{0},\left(i, l_{1}\right)\right)
$$

and

$$
\begin{aligned}
& \underline{\varliminf_{n \rightarrow \infty}} \frac{1}{n} \log \mathbb{P}_{(n-1, y)}\left(\vec{X}_{n}^{n+q_{0}-1}=\vec{x}^{0}\right) \\
& \quad \underline{\lim } \frac{1}{n}\left[\log p_{n}\left(y, x_{1}^{0}\right)+\log p_{n}\left(x_{1}^{0}, x_{2}^{0}\right)+\cdots+\log p_{n}\left(x_{q_{0}-1}^{0}, x_{q_{0}}^{0}\right)\right]=0 .
\end{aligned}
$$


Such a vector $\vec{x}^{0}$ exists from the primitivity of $P^{*}(i)$.

Similarly, form vectors $\vec{x}^{s}=\left\langle x_{1}^{s}, \ldots, x_{q_{s}}^{s}\right\rangle$ in $C_{l_{s}}$ where $1 \leq q_{s} \leq \mathfrak{r}+1$ for $1 \leq s \leq k$, and $1 \leq q_{k+1} \leq \mathfrak{r}$. Also specify that

$$
p_{n-1+r(s)+1}\left(x_{q_{s}}^{s}, x_{1}^{s+1}\right)=t\left(n-1+r(s)+1,\left(l_{s}, l_{s+1}\right)\right)
$$

for $1 \leq s \leq k$. In addition, the paths are chosen so

$$
\underline{\lim } \frac{1}{n} \log \mathbb{P}_{\left(n-1+r(s-1)+1, x_{1}^{s}\right)}\left(\vec{X}_{n+r(s-1)+1}^{n+r(s)-1}=\vec{x}_{2}^{s}\right)=0
$$

and

$$
\underline{\lim } \frac{1}{n} \log \mathbb{P}_{\left(n+r(k+1)-1, x_{q_{k+1}}^{k+1}\right)}\left(X_{n+r(k+1)}=z\right)=0
$$

when $l_{s} \in \mathcal{G}$ and $q_{s} \geq 2$, and $\vec{x}_{2}^{s}$ and $r(s)$ are as before. Then,

$$
\begin{aligned}
\underline{\lim _{n \rightarrow \infty}} \frac{1}{n} \log \underline{\gamma}^{1}(n, y, z) & \geq \underline{\lim } \frac{1}{n} \sum_{s=0}^{k+1} \log t\left(n-1+r(s),\left(l_{s}, l_{s+1}\right)\right) \\
& \geq \sum_{s=0}^{k+1} \tau\left(l_{s}, l_{s+1}\right)=\mathcal{T}_{0}(i, j) .
\end{aligned}
$$

\section{Examples}

In this section, we present three examples concerning possible LD behaviors of $\left\{Z_{n}(f)\right\}$ under $\mathbb{P}_{\pi} \in \mathbb{A}(P)$. The first shows that even if Assumption $\mathrm{A}$ is violated an LDP may still hold with respect to some processes and functions $f$. The second example shows that the bounds in Theorems 3.1 and 3.2 (ii) may be achieved. The third example shows that it is possible an LDP is non-existent under Assumption A when one of the submatrices $\{P(i): i \in \mathcal{G}\}$ is periodic and Assumptions $\mathrm{B}$ and $\mathrm{C}$ do not hold.

\subsection{Assumption A is Not Necessary for LDP}

The point is that if the connecting transition probabilities oscillate, so that Assumption A fails, but not too wildly then the process on the large deviation scale can wait an $o(n)$ time to select optimal connections. Let $\Sigma=\{0,1\}$ and initial distribution $\pi=\langle 1 / 2,1 / 2\rangle$. Let also $f: \Sigma \rightarrow \mathbb{R}$ be given by $f(0)=1$ and $f(1)=0$, and for $k \geq 1$, define transition matrices

$$
A_{k}=\left[\begin{array}{cc}
1-\left(\frac{1}{2}\right)^{k} & \left(\frac{1}{2}\right)^{k} \\
0 & 1
\end{array}\right], \quad \text { and } B_{k}=\left[\begin{array}{cc}
1-\left(\frac{1}{3}\right)^{k} & \left(\frac{1}{3}\right)^{k} \\
0 & 1
\end{array}\right] .
$$


Then, for $n \geq 1$, let

$$
P_{n}= \begin{cases}A_{n} & \text { for } n \text { even } \\ B_{n} & \text { for } n \text { odd }\end{cases}
$$

The limit matrix $P$ is the $2 \times 2$ identity matrix $I_{2}$, with two irreducible sets, $C_{0}=\{0\}$ and $C_{1}=\{1\}$. Both sets correspond to degenerate rate functions, for $i=0,1, \mathbb{I}_{i}(x)=0$ for $x=1-i$ and $=\infty$ otherwise. Also, one sees that $\tau(0,1)=-\log 3<-\log 2=$ $v(0,1)$, so Assumption A is not satisfied here. Of course, $\tau(1,0)=v(1,0)=-\infty$. Also, the process satisfies condition (SIE)-1.

To identify the large deviations of $\left\{Z_{n}(f)\right\}$ under $\mathbb{P}_{\pi}^{\left\{P_{n}\right\}}$, we focus on sets $\Gamma=(a, b]$ for $0<a<b<1$, as the analysis on other types of sets is similar.

As before, $A(0)$ and $A(1)$ are the events that $\vec{X}_{n}$ does not switch and switches exactly once between sets $C_{0}$ and $C_{1}$. Since $\Gamma$ is such that $\mathbb{P}_{\pi}\left(Z_{n} \in \Gamma, A(0)\right)=0$, and also since the chain cannot switch from state 1 to 0 , we have

$$
\mathbb{P}_{\pi}\left(Z_{n} \in \Gamma\right)=\mathbb{P}_{\pi}\left(Z_{n} \in \Gamma, A(1)\right)=\mathbb{P}_{\pi}\left(Z_{n} \in \Gamma, A(1), X_{1}=0, X_{n}=1\right) .
$$

The event $\left\{A(1), X_{1}=0, X_{n}=1\right\} \subset \Sigma^{n}$ consists exactly of $n-1$ paths $\vec{x}_{n, i}$ starting at 0 but switching to 1 at time time $1 \leq i \leq n-1$. Now compute that

$$
\begin{aligned}
\mathbb{P}_{\pi}\left(\vec{X}_{n}=\vec{x}_{n, i}\right) & =\pi(0) \prod_{k=1}^{i}\left(1-\alpha(k)^{k}\right)(\alpha(i+1))^{i+1} \prod_{l=i+2}^{n}\left(1-\alpha(l)^{l}\right) \\
& =e^{o(n)}(\alpha(i+1))^{i+1}
\end{aligned}
$$

where $\alpha(k)=1 / 2$ for $k$ even and $=1 / 3$ for $k$ odd. Also, on the path $\vec{x}_{n, i}$, we have that $Z_{n}=i / n$.

Let $G_{n}^{o}=\left\{1 \leq i \leq n: i / n \in \Gamma^{o}\right\}$. Then, by Lemma 5.1, we have

$$
\begin{aligned}
\underline{\lim } & \frac{1}{n} \log \mathbb{P}_{\pi}\left(Z_{n} \in \Gamma^{o}, A(1), X_{1}=0, X_{n}=1\right) \\
& =\underline{\lim } \max _{i \in G_{n}^{o}} \frac{1}{n} \log \mathbb{P}_{\pi}\left(\vec{X}_{n}=\vec{x}_{n, i}\right) \\
& =\underline{\lim } \max \left\{\frac{\lceil a n\rceil}{n} \log (\alpha(\lceil a n\rceil)), \frac{\lceil a n\rceil+1}{n} \log (\alpha(\lceil a n\rceil+1))\right\} \\
& =a \log \left(\frac{1}{2}\right)=-a \log (2) .
\end{aligned}
$$

Similarly, $\overline{\lim }(1 / n) \log \mathbb{P}_{\pi}\left(Z_{n} \in \bar{\Gamma}, A(1), X_{1}=0, X_{n}=1\right)=-a \log (2)$.

A related analysis works for more general $\Gamma$, and we have that $\left\{Z_{n}(f)\right\}$ satisfies a LDP with rate function

$$
\mathbb{I}(z)=\left\{\begin{array}{rl}
z \log 2 & z \in[0,1) \\
0 & z=1 \\
\infty & \text { otherwise }
\end{array}\right.
$$




\subsection{Bounds May Be Sharp in Theorems 3.1 and 3.2}

The key in this example is that the connection probabilities oscillate "unboundedly," so picking out the optimal strategy is time-dependent. As before, let $\Sigma=\{0,1\}$, $\pi=\langle 1 / 2,1 / 2\rangle$, and $f: \Sigma \rightarrow \mathbb{R}$ be given by $f(0)=1$ and $f(1)=0$. Let $\{g(n)\}$ be a fast divergent sequence of integers, $g(n) \uparrow \infty, g(n)<g(n+1)$, and $g(n-1) / g(n) \rightarrow 0$. Also, for $k \geq 1$, let

$$
P_{i}= \begin{cases}I_{2} & \text { for } 1 \leq i \leq g(2) \\ A_{i} & \text { for } g(2 k)<i \leq g(2 k+1) \\ B_{i} & \text { for } g(2 k+1)<i \leq g(2 k+2)\end{cases}
$$

where $A_{i}$ and $B_{i}$ are defined in the last subsection.

To compute the large deviations of $\left\{Z_{n}(f)\right\}$, we focus now on sets $\Gamma=(a, b) \subset[0,1]$ where $0 \leq a<b<1$. Calculations for other sets are analogous. Then, in the notation of the previous example,

$$
\underline{\lim } \frac{1}{n} \log \mathbb{P}_{\pi}\left(Z_{n} \in \Gamma\right)=\underline{\lim } \frac{1}{n} \log \mathbb{P}_{\pi}\left(Z_{n} \in \Gamma, A(1), X_{1}=0, X_{n}=1\right) .
$$

Let now $n_{k}=g(2 k+2)$ for $k \geq 1$. Then, $i / n_{k} \in \Gamma$ exactly when $\lceil g(2 k+2) a\rceil \leq$ $i \leq\lfloor g(2 k+2) b\rfloor$. Also, as

$$
\lim _{n} \frac{\lceil g(2 k+2) a\rceil}{g(2 k+2)}=a>0=\lim \frac{g(2 k+1)}{g(2 k+2)}
$$

we have for all large $k$ that $g(2 k+1)+1 \leq\lceil g(2 k+2) a\rceil \leq\lfloor g(2 k+2) b\rfloor \leq g(2 k+2)$. Note also that $P_{i}=B_{i}$ for $g(2 k+1)+1 \leq i \leq g(2 k+2)$. Hence,

$$
\begin{aligned}
& \lim \frac{1}{n_{k}} \log \mathbb{P}_{\pi}\left(Z_{n_{k}} \in \Gamma, A(1), X_{1}=0, X_{n_{k}}=1\right) \\
& =\underline{\lim } \max _{i: i / n_{k} \in \Gamma} \frac{1}{n_{k}} \log \mathbb{P}_{\pi}\left(\vec{X}_{n_{k}}=\vec{x}_{n_{k}, i}\right)=\lim \frac{\lceil g(2 n+2) a\rceil}{g(2 n+2)} \log (1 / 3)=-a \log (3) .
\end{aligned}
$$

Moreover, in fact $\underline{\lim }(1 / n) \log \mathbb{P}_{\pi}\left(Z_{n} \in \Gamma\right)=-a \log (3)$

Similarly, by considering subsequence $n_{k}=g(2 k+1)$ we get

$$
\varlimsup \frac{1}{n} \log \mathbb{P}_{\pi}\left(Z_{n} \in \bar{\Gamma}, A(1), X_{1}=0, X_{n}=1\right)=-a \log (2) .
$$

These calculations, and analogous ideas, give for any $\Gamma$ that

$$
\varlimsup \frac{1}{n} \log \mu_{\pi}\left(Z_{n} \in \Gamma^{o}\right)=-\inf _{z \in \Gamma} \overline{\mathbb{J}}(z) \quad \text { and } \quad \underline{\lim } \frac{1}{n} \log \mu_{\pi}\left(Z_{n} \in \Gamma^{o}\right)=-\inf _{z \in \Gamma^{o}} \underline{\mathbb{J}}(z)
$$

where

$$
\overline{\mathbb{J}}(z)=\left\{\begin{array}{rl}
z \log 2 & \text { for } z \in[0,1) \\
0 & z=1 \\
\infty & \text { otherwise }
\end{array} \quad \text { and } \quad \underline{J}(z)=\left\{\begin{array}{rl}
z \log 3 & \text { for } z \in[0,1) \\
0 & z=1 \\
\infty & \text { otherwise. }
\end{array}\right.\right.
$$


On the other hand, these lower and upper rate functions match those in Theorems 3.1 and 3.2 (i). As $\mathcal{T}_{0}(0,1)=-\log 3, \mathcal{U}_{0}(0,1)=-\log 2$, and $t(k,(1,0))=0$ for all $k \geq 1$, we have

$$
\begin{aligned}
\mathbb{J}_{\mathcal{T}_{0}}(z) & =-\inf _{\delta \in[0,1]} \inf _{\langle x, y\rangle \in D(2,\langle\delta, 1-\delta\rangle, z)} \min \left\{\mathbb{I}_{0}(y), \delta \log (3)+\delta \mathbb{I}_{0}(x)+(1-\delta) \mathbb{I}_{1}(y)\right\} \\
& =\underline{\mathbb{J}}(z)
\end{aligned}
$$

and analogously $\mathbb{J}_{\mathcal{U}_{0}}=\overline{\bar{J}}$.

\subsection{Periodicity and Non-Existence of LDP}

We consider a process which satisfies Assumptions A but not Assumptions B or C for which an LDP cannot hold through an explicit contradiction. Also, we show that the lowerbound with respect to $\mathcal{T}_{0}$ in Theorem 3.2 does not work for this example.

Let $\Sigma=\{1, \ldots, 9\}$ and $\pi$ be the uniform distribution on $\Sigma$. For $n$ of the form $n=1+3 j$ for $j \geq 0$, except when $n=3^{2^{j}}+1$ for $j \geq 5$, let

$$
\begin{aligned}
& \bar{P}_{n}=\left[\begin{array}{ccccccccc}
1 / 3 & 1 / 3 & 1 / 3 & 0 & 0 & 0 & 0 & 0 & 0 \\
1 / 3 & 1 / 3 & 1 / 3 & 0 & 0 & t(n,(1,2)) & 0 & 0 & 0 \\
1 / 3 & 1 / 3 & 1 / 3 & 0 & 0 & 0 & 0 & 0 & 0 \\
0 & 0 & 0 & 0 & 1 & 0 & 0 & 0 & t(n,(2,3)) \\
0 & 0 & 0 & 0 & 0 & 1 & 0 & 0 & 0 \\
0 & 0 & 0 & 1 & 0 & 0 & 0 & 0 & \underline{t}(n,(2,3)) \\
0 & 0 & 0 & 0 & 0 & 0 & 1 / 3 & 1 / 3 & 1 / 3 \\
0 & 0 & 0 & 0 & 0 & 0 & 1 / 3 & 1 / 3 & 1 / 3 \\
0 & 0 & 0 & 0 & 0 & 0 & 1 / 3 & 1 / 3 & 1 / 3
\end{array}\right] \\
& \bar{P}_{n+1}=\left[\begin{array}{ccccccccc}
1 / 3 & 1 / 3 & 1 / 3 & 0 & 0 & 0 & 0 & 0 & 0 \\
1 / 3 & 1 / 3 & 1 / 3 & 0 & 0 & 0 & 0 & 0 & 0 \\
1 / 3 & 1 / 3 & 1 / 3 & t(n+1,(1,2)) & 0 & 0 & 0 & 0 & 0 \\
0 & 0 & 0 & 0 & 1 & 0 & \underline{t}(n+1,(2,3)) & 0 & 0 \\
0 & 0 & 0 & 0 & 0 & 1 & t(n+1,(2,3)) & 0 & 0 \\
0 & 0 & 0 & 1 & 0 & 0 & 0 & 0 & 0 \\
0 & 0 & 0 & 0 & 0 & 0 & 1 / 3 & 1 / 3 & 1 / 3 \\
0 & 0 & 0 & 0 & 0 & 0 & 1 / 3 & 1 / 3 & 1 / 3 \\
0 & 0 & 0 & 0 & 0 & 0 & 1 / 3 & 1 / 3 & 1 / 3
\end{array}\right], \\
& \bar{P}_{n+2}=\left[\begin{array}{ccccccccc}
1 / 3 & 1 / 3 & 1 / 3 & 0 & t(n+2,(1,2)) & 0 & 0 & 0 & 0 \\
1 / 3 & 1 / 3 & 1 / 3 & 0 & 0 & 0 & 0 & 0 & 0 \\
1 / 3 & 1 / 3 & 1 / 3 & 0 & 0 & 0 & 0 & 0 & 0 \\
0 & 0 & 0 & 0 & 1 & 0 & 0 & 0 & 0 \\
0 & 0 & 0 & 0 & 0 & 1 & 0 & \underline{t}(n+2,(2,3)) & 0 \\
0 & 0 & 0 & 1 & 0 & 0 & 0 & t(n+2,(2,3)) & 0 \\
0 & 0 & 0 & 0 & 0 & 0 & 1 / 3 & 1 / 3 & 1 / 3 \\
0 & 0 & 0 & 0 & 0 & 0 & 1 / 3 & 1 / 3 & 1 / 3 \\
0 & 0 & 0 & 0 & 0 & 0 & 1 / 3 & 1 / 3 & 1 / 3
\end{array}\right] .
\end{aligned}
$$


For $n=3^{2^{j}}+1$ for $j \geq 5$, let $\hat{P}_{n+1}$ and $\hat{P}_{n+2}$ be defined as before, but now let

$$
\bar{P}_{n}=\left[\begin{array}{ccccccccc}
1 / 3 & 1 / 3 & 1 / 3 & 0 & 0 & 0 & 0 & 0 & 0 \\
1 / 3 & 1 / 3 & 1 / 3 & 0 & 0 & t(n,(1,2)) & 0 & 0 & 0 \\
1 / 3 & 1 / 3 & 1 / 3 & 0 & 0 & 0 & 0 & 0 & 0 \\
0 & 0 & 0 & 0 & 1 & 0 & 0 & 0 & t(n,(2,3)) \\
0 & 0 & 0 & 0 & 0 & 1 & 0 & 0 & \underline{t}(n,(2,3)) \\
0 & 0 & 0 & 1 & 0 & 0 & 0 & 0 & 0 \\
0 & 0 & 0 & 0 & 0 & 0 & 1 / 3 & 1 / 3 & 1 / 3 \\
0 & 0 & 0 & 0 & 0 & 0 & 1 / 3 & 1 / 3 & 1 / 3 \\
0 & 0 & 0 & 0 & 0 & 0 & 1 / 3 & 1 / 3 & 1 / 3
\end{array}\right]
$$

Suppose now that $t(n,(1,2)), t(n,(2,3))$ and $\underline{t}(n,(2,3))$ vanish as $n$ tends to infinity and limits

$$
\lim \frac{1}{n} \log t(n(1,2)), \quad \lim \frac{1}{n} \log t(n,(2,3)), \quad \text { and } \quad \lim \frac{1}{n} \log \underline{t}(n,(2,3))
$$

exist and equal respectively

$v(1,2)=\tau(1,2)=0, v(n,(2,3))=\tau(n,(2,3))=A$, and $\lim \frac{1}{n} \log \underline{t}(n,(2,3))=2 A+\epsilon$ where $A<0$ and $\epsilon>0$ is chosen small enough so that $2 A+\epsilon<A$.

Define the diagonal matrix $\Delta_{n}=\operatorname{diag}\left\{\lambda_{1}^{-1}, \ldots, \lambda_{9}^{-1}\right\}$ where $\lambda_{i}$ is the $i$ th row sum of $\bar{P}_{n}$. Then, $\lim \Delta_{n}=I_{9}$. Let $P_{n}=\Delta_{n} \bar{P}_{n}$ for $n \geq 1$. The limit matrix $P=\lim P_{n}=$ $\lim \bar{P}_{n}$ corresponds to three sets, $C_{1}=\{1,2,3\}, C_{2}=\{4,5,6\}$ and $C_{3}=\{7,8,9\}$.

Let also $f$ be a one dimensional function on the state space such that $f(1)=$ $f(2)=f(3)=1, f(4)=f(5)=f(6)=2$, and $f(7)=f(8)=f(9)=3$. We now will concentrate the sequence $\left\{Z_{n}(f)\right\}$ with respect to the process $\mathbb{P}_{\pi}^{\left\{P_{n}\right\}}$.

Assumptions. By inspection, it is clear that Condition (SIE)-1 and Assumption A hold, but Assumptions B and C do not hold.

Non-existence of $L D P$. First, let $\mu_{\pi}$ be the measure constructed from $\left\{\bar{P}_{n}\right\}$ and $\pi$ through $(\mathrm{CON})$. It is not difficult to see that the large deviations of $Z_{n}$ under $\mathbb{P}_{\pi}$ is the same as with respect to $\mu_{\pi}$, that is, for Borel $\Gamma \subset \mathbb{R}^{d}$,

$$
\begin{gathered}
\varlimsup \\
\varlimsup \\
\frac{\lim }{n} \frac{1}{n} \log \mathbb{P}_{\pi}\left(Z_{n} \in \bar{\Gamma}\right)=\varlimsup \\
\left.\mathbb{l}_{n} \in Z^{o}\right)=\underline{\lim } \frac{1}{n} \log \mu_{\pi}\left(Z_{n} \in \bar{\Gamma}\right), \quad \text { and } \\
\log \mu_{\pi}\left(Z_{n} \in \Gamma^{o}\right)
\end{gathered}
$$

(cf. Proposition 7.1). Second, the rate functions on the three sets are degenerate,

$$
\mathbb{I}_{i}(z)=\left\{\begin{array}{ll}
0 & \text { if } z=i \\
\infty & \text { otherwise }
\end{array} \quad \text { for } i=1,2,3 .\right.
$$

Consider now the following two lemmas proved later 
Lemma 12.1 For $0<\epsilon<1 / 2$, let $\Gamma=[2+\epsilon, 2+2 \epsilon]$. Then,

$$
\varlimsup \frac{1}{n} \log \mu_{\pi}\left(Z_{n} \in \bar{\Gamma}\right)>(1-2 \epsilon) A .
$$

Lemma 12.2 For $0<\epsilon<1 / 2$ and $\theta>0$, let $\Gamma(\theta)=(2+\epsilon-\theta, 2+2 \epsilon+\theta)$. Then,

$$
\frac{\lim }{\theta \downarrow 0} \frac{\lim }{n \rightarrow \infty} \frac{1}{n} \log \mu_{\pi}\left(Z_{n} \in \Gamma(\theta)\right) \leq(1-2 \epsilon) A .
$$

These results show that no LDP is possible. If an LDP were to hold with rate function $I$ say then

$$
\begin{aligned}
(1-2 \epsilon) A & \geq \frac{\lim }{\theta \downarrow 0} \frac{\lim }{n \rightarrow \infty} \frac{1}{n} \log \mu_{\pi}\left(Z_{n} \in \Gamma(\theta)\right) \\
& \geq \frac{\lim }{\theta \downarrow 0}-\inf _{x \in \Gamma(\theta)} I(x) \geq-\inf _{x \in \bar{\Gamma}} I(x) \geq \varlimsup_{n \rightarrow \infty} \frac{1}{n} \log \mu_{\pi}\left(Z_{n} \in \bar{\Gamma}\right)>(1-2 \epsilon) A
\end{aligned}
$$

leading to a contradiction.

Lowerbound in Theorem 3.2 (i) does not hold. Consider the following lemma proved at the end of the subsection.

Lemma 12.3 With respect to $\Gamma(\theta)$ as in Lemma 12.2, we have

$$
-\inf _{z \in \Gamma(\theta)} \mathbb{J}_{\mathcal{T}_{0}}(z)=(1-2 \epsilon-\theta) \frac{A}{2} .
$$

Then, a clear contradiction with Lemma 12.2 would entail if the lowerbound in Theorem 3.2 (ii) was valid.

Proof of Lemma 12.1. No $n$-word $\vec{x}_{n}$ remaining solely in a single closed set can have an average in $\bar{\Gamma}$. Also, by construction, no $n$-word may pass from $C_{i}$ to $C_{j}$ for $i>j$, or in one step from $C_{1}$ to $C_{3}$. Therefore, the only $n$-words such that $\frac{1}{n} \sum_{i=1}^{n} f\left(x_{i}\right) \in[2+\epsilon, 2+2 \epsilon]$ are those which visit succesively $C_{1}, C_{2}$, and $C_{3}$, or those which visit first $C_{2}$ and then $C_{3}$.

We now examine $(1 / n) \log \mu_{\pi}\left(Z_{n} \in \bar{\Gamma}\right)$ along the sequence

$$
n_{k}=\left\lceil 3^{2^{k}} /\left(\frac{1-2 \epsilon}{2}\right)\right\rceil
$$

for $k \geq 1$. Let now

$A\left(n_{k}\right)$ be the set of $n_{k^{-}}$words $\vec{x}_{n_{k}}$ which stay in $C_{1}$ until time $3^{2^{k}}$, spend one time unit in $C_{2}$, and then switch to $C_{3}$. 
By definition, for $\vec{x}_{n_{k}} \in A\left(n_{k}\right)$ and $k$ large enough, we have

$$
\begin{aligned}
\frac{1}{n_{k}} \sum_{i=1}^{n_{k}} f\left(x_{i}\right) & =\frac{3^{2^{k}}}{n_{k}}+\frac{2}{n_{k}}+\left(1-\frac{\left(3^{2^{k}}+1\right)}{n_{k}}\right) 3 \\
& \in[2+\epsilon, 2+2 \epsilon] .
\end{aligned}
$$

Then, with $\delta(\epsilon)=(1-2 \epsilon) / 2$, we have

$$
\begin{aligned}
& \varlimsup_{n \rightarrow \infty} \frac{1}{n} \log \mu_{\pi}^{n}\left(Z_{n} \in \bar{\Gamma}\right) \\
& \geq \varliminf_{k \rightarrow \infty} \frac{1}{n_{k}} \log \mu_{\pi}^{n_{k}}\left(Z_{n_{k}} \in \bar{\Gamma}, A\left(n_{k}\right)\right) \\
& \geq-\inf _{\langle x, y\rangle \in D(2,\langle\delta(\epsilon), 1-\delta(\epsilon)\rangle, \bar{\Gamma})}-\delta(\epsilon)\left\{\underline{\lim } \frac{1}{k} \log t(k,(1,2))+\underline{\lim } \frac{1}{k} \log \underline{t}(k,(2,3))\right\} \\
& +\delta(\epsilon) \mathbb{I}_{1}(x)+(1-\delta(\epsilon)) \mathbb{I}_{3}(y) \\
& =\delta(\epsilon)\left\{\lim \frac{1}{k} \log t(k,(1,2))+\lim \frac{1}{k} \log \underline{t}(k,(2,3))\right\}-\delta(\epsilon) \mathbb{I}_{1}(1)-(1-\delta(\epsilon)) \mathbb{I}_{3}(3) \\
& =\frac{1-2 \epsilon}{2}(2 A+\epsilon)>(1-2 \epsilon) A \text {. }
\end{aligned}
$$

Proof of Lemma 12.2. Let now $n_{k}=3^{2^{k}}$ for $k \geq 1$. We first show that $\vec{x}_{n_{k}}$ cannot visit $C_{1}, C_{2}$, and $C_{3}$ in succession, and satisfy $\frac{1}{n_{k}} \sum_{i=1}^{n_{k}} f\left(x_{i}\right) \in \Gamma(\theta)$ for all small $\theta$. Indeed, by construction, a path $\vec{x}_{n_{k}}$ which visits $C_{1}, C_{2}$ and $C_{3}$, must switch from $C_{2}$ to $C_{3}$ at a time less or equal to $3^{2^{k-1}}$. But then, as $f(\cdot) \geq 1$ and $3^{2^{k-1}} / n_{k} \rightarrow 0$, we have for large $k$ and $\theta$ sufficiently small that

$$
\frac{1}{n_{k}} \sum_{i=1}^{n_{k}} f\left(x_{i}\right) \geq \frac{3^{2^{k-1}}}{n_{k}}+\left(1-\frac{3^{2^{k-1}}+1}{n_{k}}\right) 3>2+2 \epsilon+\theta .
$$

Thus, if $\vec{x}_{n_{k}} \in \Gamma(\theta)$, we deduce $\vec{x}_{n_{k}}$ begins in $C_{2}$ and then switches to $C_{3}$. Let now $\tau(\epsilon)=1-2 \epsilon$. We have

$$
\begin{aligned}
& \underline{\lim _{\theta \downarrow}} \varliminf_{n \rightarrow \infty} \frac{1}{n} \log P_{\pi}\left(Z_{n} \in \Gamma(\theta)\right)
\end{aligned}
$$

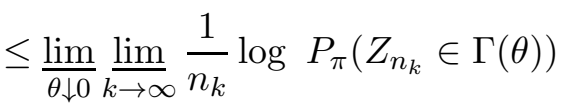

$$
\begin{aligned}
& =\underline{\lim _{\theta \downarrow 0}} \sup _{0 \leq \delta \leq 1} \sup _{\langle x, y\rangle \in D(2,\langle\delta, 1-\delta\rangle, \Gamma(\theta))} \delta \varlimsup \frac{1}{k} \log t(k,(2,3))-\delta \mathbb{I}_{2}(x)-(1-\delta) \mathbb{I}_{3}(y) \\
& =\tau(\epsilon) \varlimsup \frac{1}{k} \log t(k,(2,3))=(1-2 \epsilon) A
\end{aligned}
$$

as $\tau(\epsilon)$ is the smallest $\delta$ such that $(2,3) \in D(2,\langle\delta, 1-\delta\rangle,[2-\epsilon, 2+2 \epsilon])$. 
Proof of Lemma 12.3. Since motion is possible only from $C_{1}$ to $C_{2}$ to $C_{3}$, and the corresponding rate functions are degenerate at $x_{1}=1, x_{2}=2$ and $x_{3}=3$, we have

$$
\begin{aligned}
\mathbb{J}_{\mathcal{T}_{0}}(\Gamma(\theta)) & =\sup _{\substack{v_{1}+v_{2}+v_{3}=1 \\
0 \leq v_{1}, v_{2}, v_{3} \leq 1}} \sup _{\substack{x \in D(3, \vec{v}, \Gamma(\theta)) \\
v_{1}+2 v_{2}+3\left(1-v_{1}-v_{2}\right) \in \Gamma(\theta) \\
0 \leq v_{1}, v_{2} \leq 1}}\left(v_{1}+v_{2}\right) A=(1-2 \epsilon-\theta)(A / 2) . \\
& v_{i=1}^{3} v_{i} \mathbb{I}_{i}\left(x_{i}\right) \\
&
\end{aligned}
$$

\section{Appendix}

\subsection{Proof of Lemma 4.1}

We consider separately the situations when $0<\delta<1$ and $\delta=0$.

Case $\delta>0$. Let $\hat{t}_{n}=\sup _{s \geq n} t_{s}$. Then, $t_{n} \leq \hat{t}_{n}, 0<\hat{t}_{n} \leq 1$ and $\hat{t}_{n} \downarrow \delta$. Also,

$$
\lim \frac{1}{n} \log \hat{t}_{n} \rightarrow 0=\varlimsup \frac{1}{n} \log t_{n} .
$$

Case $\delta=0$. The proof is split into two subcases.

Subcase 1: $\varlimsup(1 / n) \log t_{n}=t<0$. If $t_{n}$ vanishes eventually, that is $t_{n}=0$ for $n \geq N_{0}$, some $N_{0} \geq 1$, then we may take

$$
\hat{t}_{n}=\left\{\begin{aligned}
1 & \text { for } 1 \leq n<N_{0} \\
e^{-n^{2}} & \text { for } n \geq N_{0}
\end{aligned}\right.
$$

Otherwise, let $a_{n}=\sup _{j \geq n}(1 / j) \log t_{j}$ and $\hat{t}_{n}=\exp \left\{\sup _{l \geq n} l a_{l}\right\}$. Note $a_{n} \downarrow t$, and $\hat{t}_{n} \geq \exp \left\{n a_{n}\right\} \geq \exp \left\{n(1 / n) \log t_{n}\right\}=t_{n}$, and also that $1 \geq \hat{t}_{n}>0$. In addition, $(1 / n) \log \hat{t}_{n} \geq a_{n} \rightarrow t$.

Let now $1>\epsilon>0$ and let $N_{1}$ be such that $a_{n}<(1-\epsilon) t$ for $n \geq N_{1}$. Then,

$$
\frac{1}{n} \log \hat{t}_{n} \leq \frac{1}{n} \sup _{l \geq n} l t(1-\epsilon)=t(1-\epsilon)
$$

for $n \geq N_{1}$. As $\epsilon$ is arbitrary, we then have $(1 / n) \log \hat{t}_{n} \rightarrow t$.

Subcase 2: $\overline{\lim }(1 / n) \log t_{n}=t=0$. As $t_{n} \rightarrow 0$, we have $t_{n}<1$ for $n \geq N_{2}$ say. Let $b_{j}=\max _{N_{2} \leq l \leq j}(1 / l) \log t_{l}$ for $j \geq N_{2}$, and let

$$
\hat{t}_{n}=\left\{\begin{aligned}
1 & \text { for } n<N_{2} \\
\exp \left\{\sup _{j \geq n} j b_{j}\right\} & \text { for } n \geq N_{2} .
\end{aligned}\right.
$$

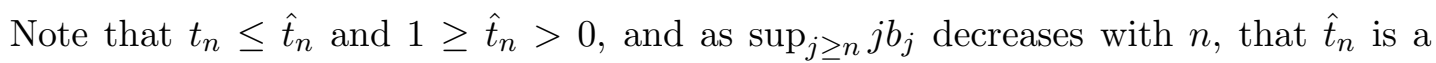
decreasing sequence. 
We now identify the limit. Note that $b_{j} \leq 0$ for all $j \geq N_{2}$ and $(1 / l) \log t_{l} \rightarrow 0$. Then, for each $K \geq N_{2}$, there is an index $J_{K} \geq K$ such that

$$
b_{j}=\max _{K \leq l \leq j}(1 / l) \log t_{l} \text { for } j \geq J_{K} .
$$

Hence, for large $n$, and given $K \geq N_{2}$,

$$
\hat{t}_{n}=\exp \left\{\sup _{j \geq n} j \max _{K \leq l \leq j}(1 / l) \log t_{l}\right\} \leq \exp \left\{\sup _{j \geq n} \max _{K \leq l \leq j} \log t_{l}\right\}=\sup _{j \geq n} \max _{K \leq l \leq j} t_{l} .
$$

As $K$ is arbitrary, we have that $\hat{t}_{n} \downarrow 0$.

Finally, as $b_{j} \rightarrow 0$, we have for $\epsilon>0$ and large $n$ that

$$
0 \geq(1 / n) \log \hat{t}_{n}=(1 / n) \sup _{j \geq n} j b_{j} \geq(1 / n) \sup _{j \geq n} j(-\epsilon)=-\epsilon .
$$

As $\epsilon$ is arbitrary, we have $(1 / n) \log \hat{t}_{n} \rightarrow 0$.

\subsection{An Extended Gärtner-Ellis Theorem}

We give here a minor extension of the Gärtner-Ellis theorem and state some general conditions under which a sequence of bounded non-negative measures $\left\{\mu_{n}\right\}$ on $\mathbb{R}^{d}$ satisfies an LDP.

For $\lambda \in \mathbb{R}^{d}$, define the extended real sequence $\Lambda_{n}(\lambda)=\log \int_{\mathbb{R}^{d}} e^{\langle\lambda, x\rangle} d \mu_{n}(x)$ and also $\Lambda(\lambda)=\lim _{n \rightarrow \infty}(1 / n) \Lambda_{n}(n \lambda)$ provided the extended limit exists. We now recall when $\Lambda$ is essential smoothness (cf. [10]).

\section{Assumption E}

1. For all $\lambda \in \mathbb{R}^{d}, \Lambda(\lambda)$ exists as an extended real number in $(-\infty, \infty]$.

2. Let $D_{\Lambda}=\{\lambda:-\infty<\Lambda(\lambda)<\infty\}$. Suppose $0 \in D_{\Lambda}^{o}$.

3. $\Lambda(\cdot)$ is differentiable throughout $D_{\Lambda}^{o}$.

4. When $\left\{\lambda_{n}\right\} \subset D_{\Lambda}^{o}$ converges to a boundary point of $D_{\Lambda}$, we have $\left|\nabla \Lambda\left(\lambda_{n}\right)\right| \rightarrow \infty$.

5. $\Lambda(\lambda)$ is a lower semi-continuous function.

We now state the standard Gärtner-Ellis theorem (cf. [10]).

Proposition 13.1 Let $\left\{\nu_{n}\right\}$ be a sequence of probability measures which satisfy Assumption E. Let $\mathbb{I}$ be the Legendre transform of $\Lambda$. Then, $\mathbb{I}$ is a rate function and $\left\{\nu_{n}\right\}$ satisfies $L D P$ (2.1).

The main result of the subsection is the following.

Proposition 13.2 Let $\left\{\mu_{n}\right\}$ be a sequence of bounded non-negative measures on $\mathbb{R}^{d}$ satisfying Assumption E. Let $\mathbb{I}$ be the Legendre transform of $\Lambda$. Then, $\mathbb{I}$ is an extended rate function and the $L D P$ (2.1) holds.

Moreover, $\mathbb{I}$ can be decomposed as the difference of a rate function of a probability sequence and a constant, $\mathbb{I}=\mathbb{I}^{1}-\Lambda(0)$. 
Proof. By Assumption E, with $\lambda=0$, we have that $(1 / n) \log \mu_{n}\left(\mathbb{R}^{d}\right) \rightarrow \Lambda(0) \in \mathbb{R}$. Consider now, the probability measures $\nu_{n}(\cdot)=\mu_{n}(\cdot) / \mu_{n}\left(\mathbb{R}^{d}\right)$. The pressure of the sequence $\left\{\nu_{n}\right\}$ is calculated as $\Lambda(\cdot)-\Lambda(0)$. Since Assumption E holds for $\Lambda(\cdot)$, it also holds for the shifted function $\Lambda(\cdot)-\Lambda(0)$. Therefore, by Proposition 13.1, we have that $\left\{\nu_{n}\right\}$ satisfies $(2.1)$ with rate function $\mathbb{I}^{1}$ given by

$$
\mathbb{I}^{1}(x)=\sup _{\lambda}\{\langle\lambda, x\rangle-(\Lambda(\lambda)-\Lambda(0))\}=\sup _{\lambda}\{\langle\lambda, x\rangle-\Lambda(\lambda)\}+\Lambda(0) .
$$

Let now $\mathbb{I}(x)=\sup _{\lambda}\{\langle\lambda, x\rangle-\Lambda(\lambda)\}$, so that $\mathbb{I}=\mathbb{I}^{1}-\Lambda(0)$. As $\mu_{n}(\cdot)=\mu_{n}\left(\mathbb{R}^{d}\right) \nu_{n}(\cdot)$, by translating we obtain that $(2.1)$ holds for $\left\{\mu_{n}\right\}$ sequence with rate function $\mathbb{I}$.

\subsection{Proof of Proposition 2.1}

Extended pressure $\Lambda$. We follow the method in [10] to identify the extended pressure of the sequence $\left\{\mu_{n}\right\}$.

$$
\begin{aligned}
\Lambda(\lambda) & =\lim \frac{1}{n} \log \Lambda_{n}(n \lambda) \\
& =\lim \frac{1}{n} \log \int_{\vec{X}_{n} \in C^{n}} e^{\left\langle\lambda, \sum f\left(X_{i}\right)\right\rangle} d \mathbb{U}_{\pi}=\lim \frac{1}{n} \log \left(\pi^{t}\left(\Pi_{C, \lambda}\right)^{n} \overrightarrow{1}\right) .
\end{aligned}
$$

Since $\Pi_{C, \lambda}$ is an irreducible matrix, the Perron-Frobenius eigenvalue $\rho(C, \lambda)$ possesses a right Perron-Frobenius eigenvector $\vec{v}(\lambda)$ with positive entries. Let $a$ and $b$ be the smallest and largest entries. Then,

$$
\log \left(\pi^{t}\left(\Pi_{C, \lambda}\right)^{n} \overrightarrow{1}\right) \leq \log \left((1 / a) \pi^{t}\left(\Pi_{C, \lambda}\right)^{n} \vec{v}\right)=\frac{1}{n} \log \left(\frac{1}{a} \pi^{t} \vec{v}\right)+\log \rho(C, \lambda)
$$

and similarly, $\log \left(\pi^{t}\left(\Pi_{C, \lambda}\right)^{n} \overrightarrow{1}\right) \geq \log \rho(C, \lambda)+o(1)$. Hence,

$$
\Lambda(\lambda)=\lim \frac{1}{n} \log \Lambda_{n}(n \lambda)=\log \rho(C, \lambda) .
$$

Analyticity, convexity and essential smoothness of $\Lambda$. Perron-Frobenius theory guarantees that $\rho(\lambda)$ has multiplicity 1 and is positive for all $\lambda \in \mathbb{R}^{d}$. Then, by Theorem 7.7.1 $[21], \rho(\cdot)$ is analytic and so $\Lambda(\cdot)$ is analytic. Now, as $\Lambda(\lambda)$ is the limit of a sequence of convex functions, it is convex. Finally, by the comments of Section 3.1 [10], we have that $\Lambda$ is essentially smooth.

$\mathbb{I}$ is an extended rate function and $\left\{\mu_{n}\right\}$ satisfies an $L D P$. Recall now that $\mathbb{I}=\mathbb{I}_{C}$ is the Legendre transform $\mathbb{I}(x)=\sup _{\lambda \in \mathbb{R}^{d}}\langle\lambda, x\rangle-\Lambda(\lambda)$. By Proposition 13.2, we have that $\mathbb{I}$ is an extended rate function and $\left\{\mu_{n}\right\}$ satisfies an LDP with respect to $\mathbb{I}$.

$\mathbb{I}$ is a rate function when $U_{C}$ is substochastic. When $U_{C}$ is substochastic, we have $\Lambda(0) \leq 0$. Hence, by Proposition 13.2, $\mathbb{I}=\mathbb{I}^{1}-\Lambda(0) \geq 0$ and so is a rate function. 
$\mathbb{I}$ is not identically $\infty$. Let $\hat{x}=\nabla \Lambda(0)$. Then, by Theorem 23.5 [26],

$$
\mathbb{I}(\hat{x})=\sup _{\lambda \in \mathbb{R}^{d}}\langle\lambda, \nabla \Lambda(0)\rangle-\Lambda(\lambda)=\langle 0, \nabla \Lambda(0)\rangle-\Lambda(0)=-\Lambda(0)<\infty .
$$

Convexity of $\mathbb{I}$ and strict convexity on the relative interior of $Q_{C}$. As $\Lambda$ is convex, the Legendre transform $\mathbb{I}$ is convex. Also, as $\Lambda(\cdot)$ is real-valued and lower semicontinuous, by Lemma 4.5 .8 of [10], $\Lambda$ is the conjugate of $\mathbb{I}$. As $\mathbb{I}$ is not identically $\infty$, it is a proper convex function. Moreover, since $\mathbb{I}$ is lower semicontinuous, it is a closed convex function as well (cf. p. $52[26]$ ). Then, since $\Lambda$ is essentially smooth, we have from Theorem 26.3 of [26] that $\mathbb{I}$ is strictly convex on the relative interior of its domain of finiteness $Q_{C}$.

$Q_{C}$ is convex and $Q_{C} \subset \mathbb{K}$. Let $x, y \in Q_{C}$. The convexity of $\mathbb{I}$ implies that $\mathbb{I}((x+y) / 2) \leq(\mathbb{I}(x)+\mathbb{I}(y)) / 2<\infty$. Hence, $Q_{C}$ is convex.

For $\lambda \in \mathbb{R}^{d}$, let $\bar{\lambda}=\left\langle\left|\lambda_{1}\right|, \ldots,\left|\lambda_{d}\right|\right\rangle$. Then,

$$
\exp \left\langle-\bar{\lambda},\left(\max _{i}|f(i)|\right) \overrightarrow{1}_{d}\right\rangle P_{C} \leq \Pi_{C, \lambda} \leq \exp \left\langle\bar{\lambda},\left(\max _{i}|f(i)|\right) \overrightarrow{1}_{d}\right\rangle P_{C}
$$

As the Perron-Frobenius value of $P_{C}$ is 1 , we have

$$
\exp \left\langle-\bar{\lambda},\left(\max _{i}|f(i)|\right) \overrightarrow{1}_{d}\right\rangle \leq \rho(\lambda) \leq \exp \left\langle\bar{\lambda},\left(\max _{i}|f(i)|\right) \overrightarrow{1}_{d}\right\rangle .
$$

Now let $x$ be such that $x_{j}>\max _{i}|f(i)|$ for some $1 \leq j \leq d$. Then, for $\alpha \in \mathbb{R}$, let $\lambda^{j, \alpha} \in \mathbb{R}^{d}$ be such that $\lambda_{i}^{j, \alpha}=0$ for $i \neq j$ and $\lambda_{j}^{j, \alpha}=\alpha$. We have then

$$
\begin{aligned}
\mathbb{I}(x) & \geq \sup _{\lambda \in \mathbb{R}^{d}}\langle\lambda, x\rangle-\left\langle\bar{\lambda},\left(\max _{i}\left|f_{i}\right|\right) \overrightarrow{1}_{d}\right\rangle \\
& \geq\left\langle\lambda^{j, \alpha}, x\right\rangle-\left\langle\overline{\lambda^{j, \alpha}},\left(\max _{i}\left|f_{i}\right|\right) \overrightarrow{1}_{d}\right\rangle \geq \alpha x_{j}-|\alpha| \max _{i}\left|f_{i}\right| .
\end{aligned}
$$

By taking $\alpha \uparrow \infty$, we have that $\mathbb{I}(x)=\infty$. Similarly, if $x_{j}<-\max _{i}\left|f_{i}\right|$, then $\mathbb{I}(x)=\infty$. Thus, $\mathbb{I}(x)<\infty$ implies $\max _{i}\left|x_{i}\right|<\max _{i}\left|f_{i}\right|$, and so $Q_{C} \subset \mathbb{K}$.

$Q_{C}$ is compact. If $\mathbb{I}$ can be shown to be uniformly bounded on $Q_{C}$, then the lower semicontinuity of $\mathbb{I}$ will imply that $Q_{C}$ is closed. Also, since it was shown above that $Q_{C}$ is bounded, $Q_{C}$ will then be compact.

Let $\underline{p}$ be the smallest positive entry in $P_{C}$, and $G=\{x: \mathbb{I}(x) \leq-\log \underline{p}\}$. By the lower semi-continuity of $\mathbb{I}, G$ is a closed set. Let $x_{0} \in G^{c}$. We show that $\mathbb{I}\left(x_{0}\right)=\infty$, and hence $Q_{C} \subset G$.

Since $G^{c}$ is open, Let $B=\bar{B}\left(x_{0} ; \delta\right) \subset G^{c}$ be a closed ball around $x_{0}$ with some radius $\delta>0$. If now $\varlimsup \lim (1 / n) \log \mu_{n}\left(Z_{n} \in B\right)>-\infty$, then there exists a sequence $\left\{\vec{x}_{n_{k}}\right\}$ such that $\sum_{i=1}^{n_{k}} f\left(x_{i}\right) / n_{k} \in B$ and $\mu_{n}\left(\vec{X}_{n}=\vec{x}_{n_{k}}\right)>0$. But, we have $\mu_{n}\left(\vec{X}_{n}=\vec{x}_{n_{k}}\right) \geq \underline{p}^{n_{k}}$, and so $\lim \left(1 / n_{k}\right) \log \mu_{n}\left(Z_{n_{k}} \in B\right) \geq \log \underline{p}$. Hence, using the LD upper bound,

$$
-\mathbb{I}(B) \geq \varlimsup \frac{1}{n} \log \mu_{n}\left(Z_{n} \in B\right) \geq \log \underline{p} .
$$


But, since $\mathbb{I}$ is lower semi-continuous, $\mathbb{I}(B)=\mathbb{I}\left(x_{1}\right)$ on some point $x_{1}$ in the compact set $B \subset G^{c}$. Hence, $\mathbb{I}(B)>-\log \underline{p}$, giving a contradiction.

Therefore, we must have $\mathbb{I}\left(x_{0}\right)=\infty$ as

$$
-\infty=\varlimsup \frac{1}{n} \log \mu_{n}\left(Z_{n} \in B\right) \geq \underline{\lim } \frac{1}{n} \log \mu_{n}\left(Z_{n} \in B\right) \geq-\mathbb{I}\left(B^{o}\right) \geq-\mathbb{I}\left(x_{0}\right) .
$$

$\mathbb{I}$ is uniformly continuous on $Q_{C}$. As $\mathbb{I}$ is convex, $\mathbb{I}$ restricted to $Q_{C}$ is continuous. Since $Q_{C}$ is compact, $\mathbb{I}$ is in fact uniformly continuous on $Q_{C}$.

$\mathbb{I}$ is a good rate function. As $\mathbb{I}$ is lower semicontinuous, the level set $\{x: \mathbb{I}(x) \leq a\}$ for $a \in \mathbb{R}$ is a closed subset of $Q_{C}$ and hence compact.

\subsection{Proof of Proposition 4.1}

When $M=1, P\left(\zeta_{1}\right)$ is stochastic and $\mathbb{J}_{U}=\mathbb{I}_{\zeta_{1}}$, and the proof follows from Proposition 2.1. Suppose now that $M \geq 2$. Consider that $\mathbb{J}_{U} \leq \min \left\{\mathbb{I}_{i}: i \in \mathcal{G}\right\}$ and so $Q_{\mathbb{J}_{U}} \supset$ $\cup_{i \in \mathcal{G}} Q_{i}$ is non-empty. Also, $Q_{\mathbb{J}_{U}} \subset \mathbb{K}$ : Indeed, for $z \notin \mathbb{K}$ and any $\vec{v} \in \Omega_{M}$ and $\vec{x} \in D(M, \vec{v}, z)$ we must have that $v_{i}>0$ and $x_{i} \notin \mathbb{K}$ for some $1 \leq i \leq M$. Then, $C_{\vec{v}, U}(\sigma, \vec{x})=\infty$ and so $\mathbb{J}_{U}(z)=\infty$.

In addition, $\mathbb{J}_{U}$ is lower semicontinuous and non-negative as $\left\{\mathbb{I}_{i}\right\}$ corresponding to substochastic matrices $\{P(i): i \in \mathcal{G}\}$ are rate functions with compact domains of finiteness. Finally, $\mathbb{J}_{U}$ is a good rate function from the same argument given for Proposition 2.1.

Acknowledgement. We thank the referee for careful remarks which led to improvements in the article, and also for pointing out useful references. Also, we thank J. Schmalian for some helpful conversations.

\section{References}

[1] Baxter, J.R., Jain, N.C., Seppäläinen, T.O. (1993) Large deviations for nonstationary arrays and sequences. Illinois J. Math. 37, no. 2 302-328.

[2] Bovier, A., Eckhoff, M., Gayard, V., Klein, M. (2001) Metastability in stochastic dynamics of disordered mean-field models. Probab. Theory Related Fields 119 99161.

[3] Bovier, A., and Gayard, V. (1999) Sample path large deviations for a class of Markov chains related to disordered mean field models. pre-print arXiv:math.PR/9905022 v1.

[4] Bovier, A., and Manzo, F. (2002) Metastability in Glauber dynamics in the lowtemperature limit: Beyond exponential asymptotics. J. Stat. Phys. 107 757-779.

[5] Bovier, A., and Picco, P. (1998) Mathematical Aspects of Spin Glasses and Neural Networks. Ed. A. Bovier and P. Picco, Progress in Probability 41, Birkhäuser, Boston. 
[6] Catoni, O. (1999) Simulated annealing algorithms and Markov chains with rare transitions. Springer Lecture Notes in Math. 1709 69-119.

[7] Catoni, O. (1992) Rough large deviation estimates for simulated annealing: application to exponential schedules. Ann. Probab. 20 1109-1146.

[8] Catoni, O. (1991) Sharp large deviations estimates for simulated annealing algorithms. Ann. Inst. H. Poincare 27 291-382.

[9] Catoni, O., and Cerf, R. (1997) The exit path of a Markov chain with rare transitions. ESAIM Probab. Statist. 1 95-144.

[10] Dembo, A., Zeitouni, O. (1998) Large Deviations Techniques and Applications. Springer-Verlag, New York.

[11] Deuschel, J.-D., and Stroock, D. (1989) Large Deviations. AMS-Chelsea, Providence, R.I.

[12] Ellis, R. (1985) Entropy, Large Deviations, and Statistical Mechanics. SpringerVerlag, New York.

[13] Freidlin, M. I., Wentzell, A. D. (1998) Random perturbations of dynamical systems. Second edition. Grundlehren der Mathematischen Wissenschaften, 260, SpringerVerlag, New York.

[14] Gaudron, I., and Trouvé, A. (1998) Fluctuations of empirical means at low temperature for finite Markov chains with rare transitions in the general case. Probab. Theory Related Fields. 111 215-251.

[15] Geman, D. (1990) Random fields and inverse problems in imaging. Springer Lecture Notes in Math. 1427 113-193.

[16] Gidas, B. (1995) Metropolis-type Monte Carlo simulation algorithms and simulated annealing. Topics in contemporary probability and its applications, Probab. Stochastics Ser., CRC, Boca Raton, FL, 159-232.

[17] Hanen, A. (1963) Théorèmes limites pour une suite de châines de Markov. Ann. Inst. H. Poincaré 18 197-301.

[18] den Hollander, F., Olivieri, E., Scoppola, E. (2000) Metastability and nucleation for conservative dynamics. Probabilistic techniques in equilibrium and nonequilibrium statistical physics. J. Math. Phys. 41 1424-1498.

[19] Isaacson, D.L., Madsen, R.W. (1976) Markov Chains Theory and Applications. John Wiley and Sons, New York.

[20] Kifer, J. (1996) Perron-Frobenius theorem, large deviations, and random perturbations in random environments. Math. Z. 222 677-698.

[21] Lancaster, P. (1969) Theory of Matrices. Academic Press, New York.

[22] van Laarhoven, P.J.M, Aarts, E.H.L. (1987) Simulated Annealing. D. Reidel Publishing, Dordrecht. 
[23] Lewis, L.J. (1991) Atomic dynamics through the glass transition. Phys. Rev. B 44 4245-4254.

[24] Mezard, M. (2002) Statistical physics of the glass phase. Phys. A 306 25-38.

[25] Miclo, L. (1998) Sur les temps d'occupations des processus de Markov finis inhomogénes á basse tempèrature. Stochastics Stochastics Rep. 63 65-137.

[26] Rockafellar, R. T. Convex Analysis. Princeton University Press, Princeton, 1970.

[27] Salamon, P., Sibani, P., Frost, R. (2002) Facts, Conjectures and Improvements for Simulated Annealing. SIAM Monographs on Mathematical Modeling and Computation, Philadelphia.

[28] Schon, J., Sibani, P. (2000) Energy and entropy of metastable states in glassy systems. Europhysics Letters 49 196-202.

[29] Seneta, E. (1981) Non-negative Matrices and Markov Chains. Springer-Verlag, New York.

[30] Seppäläinen, T. (1993) Large deviations for lattice systems. II. Nonstationary independent fields. Probab. Theory Related Fields 97 103-112.

[31] Seppäläinen, T. (1994) Large deviations for Markov chains with random transitions. Ann. Probab. 22 713-748.

[32] Seppäläinen, T. (1995) Entropy, limit theorems, and variational principles for disordered lattice systems. Comm. Math. Phys. 171 233-277.

[33] Vares, M.E. (1996) Large deviations and metastability. Disordered systems (Temuco, 1991/1992), Travaux en Cours, Hermann, Paris 53, 1-62.

[34] Wen, L., Weiguo, Y. (1996) An extension of Shannon-McMillian theorem and some limit properties for nonhomogeneous Markov chains. Stochastic Processes and their Applications 61 129-145.

[35] Winkler, G. (1995) Image Analysis, Random Fields and Dynamic Monte Carlo Methods. Springer-Verlag, New York.

Zach Dietz

Department of Mathematics

Tulane University

6823 St. Charles Ave.

New Orleans, LA 70118

zdietz@math.tulane.edu
Sunder Sethuraman

Department of Mathematics

Iowa State University

400 Carver Hall

Ames, IA 50011

sethuram@iastate.edu

http://www.math.iastate.edu/sethuram/ 